\title{
Measurement Science Roadmap for Prognostics and Health Management for Smart Manufacturing Systems
}

\author{
Prepared for \\ Brian A. Weiss \\ National Institute of Standards and Technology \\ Gaithersburg MD 20899
}

Prepared by

Joan Pellegrino

Mauricio Justiniano

Anand Raghunathan

Energetics Incorporated

Columbia MD 21046

This publication is available free of charge from:

http://dx.doi.org/10.6028/NIST.AMS.100-2

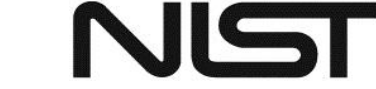

National Institute of Standards and Technology

U.S. Department of Commerce 



\title{
Measurement Science Roadmap for Prognostics and Health Management for Smart Manufacturing Systems
}

\author{
Prepared for \\ Brian A. Weiss \\ National Institute of Standards and Technology \\ Gaithersburg MD 20899
}

Prepared by

Joan Pellegrino

Mauricio Justiniano

Anand Raghunathan

Energetics Incorporated

This publication is available free of charge from: http://dx.doi.org/10.6028/NIST.AMS.100-2

September 2016

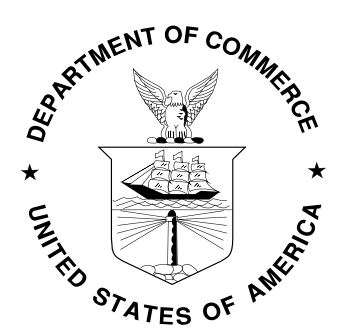

U.S. Department of Commerce

Penny Pritzker, Secretary

National Institute of Standards and Technology Willie May, Under Secretary of Commerce for Standards and Technology and Director 



\section{Photo Credits}

Report body:

Dollar Photo Club (DPC), 64341250 (pg.6), 68012349 (pg. 8), 65898405 (pg.16), 65797175 (pg.26), 74486360 (pg. 38)

\section{DISCLAIMER}

This report was prepared as an account of work cosponsored by NIST. The views and opinions expressed herein do not necessarily state or reflect those of NIST. Certain commercial entities, equipment, or materials may be identified in this document in order to illustrate a point or concept. Such identification is not intended to imply recommendation or endorsement by NIST, nor is it intended to imply that the entities, materials, or equipment are necessarily the best available for the purpose. 


\section{Acknowledgements}

This report is based on the results of the Roadmapping Workshop: Measurement Science for Prognostics and Health Management of Smart Manufacturing Systems, held November 19-20, 2014, in Gaithersburg, Maryland. The workshop was sponsored by the National Institute of Standards and Technology (NIST), an agency of the U.S. Department of Commerce.

Thanks are extended to the NIST organizers as well as the speakers and panelists who provided their perspectives on new technologies as well as the challenges impacting prognostics and health management (PHM) for smart manufacturing systems. We also appreciate the extensive contributions of the participants (listed in Appendix A); this report would not be possible without their valuable insights. Finally, thanks are extended to the Energetics Incorporated team for their assistance in facilitating the workshop and preparing this report.

Plenary Speakers (in order of appearance)

Howard Harary, NIST

Brian A. Weiss, NIST

Al Salour, The Boeing Company

Albert Wavering, NIST

Jay Lee, University of Cincinnati/Center for Intelligent Maintenance Systems

Panel 1: PHM Capabilities, Best Practices, Challenges, and Needs

Moderator: Gregory Vogl, NIST

Andrew Inman, Toyota

Carl Byington, Impact Technologies/Sikorsky

William Marscher, Mechanical Solutions, Inc.

David Siegel, Predictronics Corporation

\section{Panel 2: PHM Performance Assessment - Monitoring and Measurement}

Moderator, Moneer Helu, NIST

Harry Kekedjian, Ford Motor Company

Kai Goebel, National Aeronautics and Space Administration (NASA)

William Sobel, System Insights

John Oskin, Sage Clarity

\section{Panel 3: PHM and the Human Element}

Moderator: Patrick Brown, University of Cincinnati

Thomas Mooney, SOAR Engineering LLC

Andrew Hess, The Hess PHM Group

\section{NIST Organizers}

Brian A. Weiss, NIST

Gregory Vogl, NIST

Moneer Helu, NIST 


\section{Table of Contents}

Acknowledgements vi

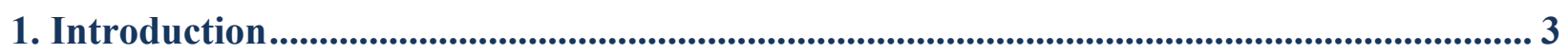

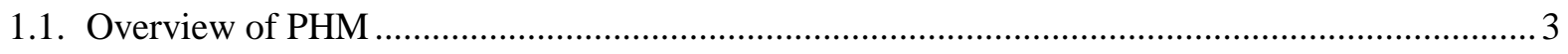

1.2. Background and Importance of Prognostics and Health Management ....................................... 3

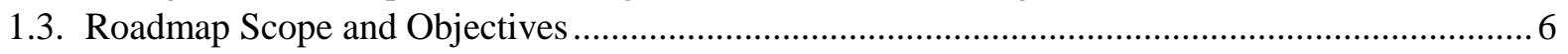

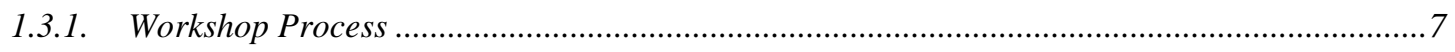

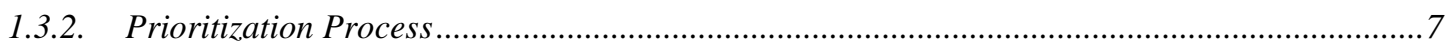

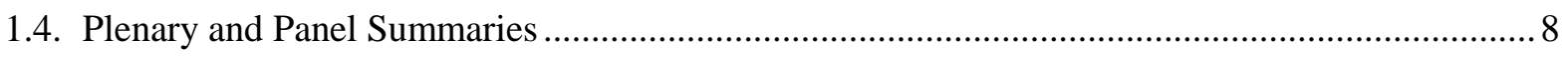

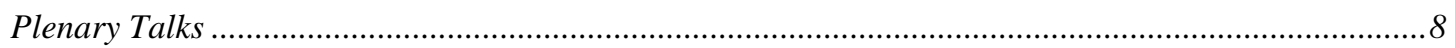

Panel 1: PHM Capabilities, Best Practices, Challenges, and Needs.................................................. 10

Panel 2: Performance Assessment - Monitoring and Measurement...................................................12

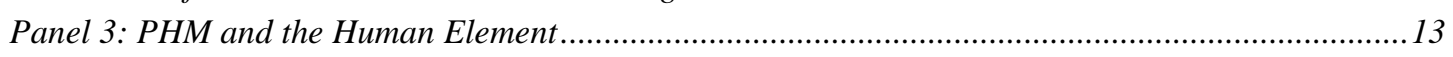

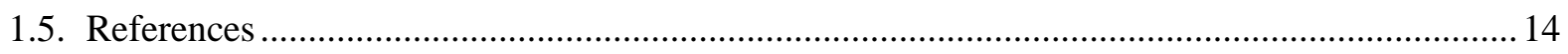

2. PHM Manufacturing Process Techniques and Metrics ...................................................... 16

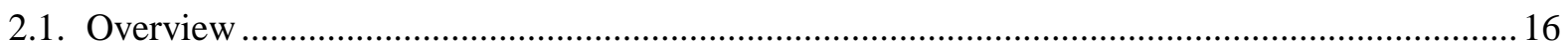

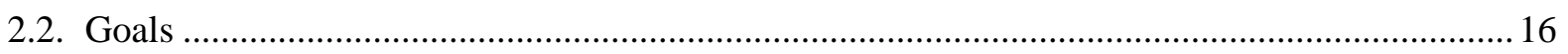

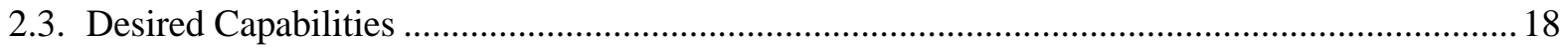

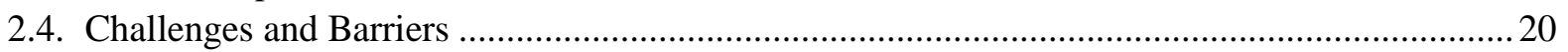

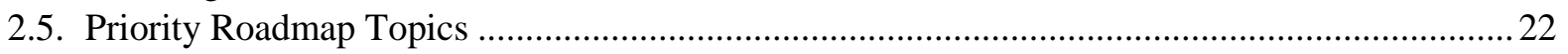

3. PHM Performance Assessment ..................................................................................................... 26

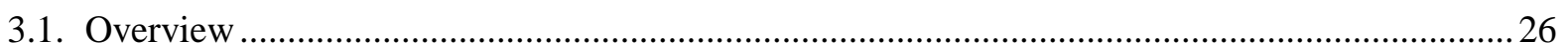

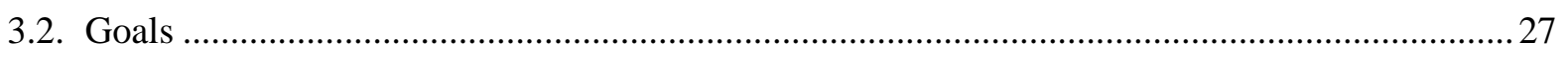

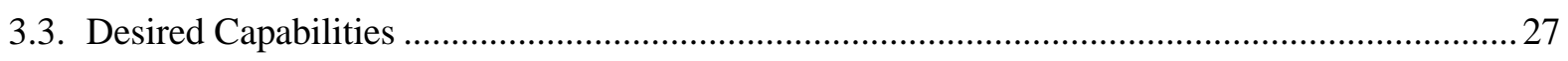

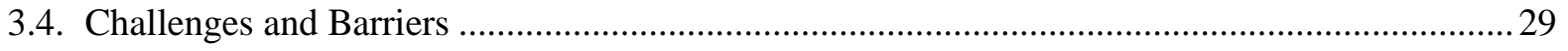

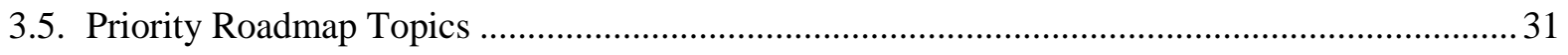

4. PHM Infrastructure - Hardware, Software, and System Integration............................... 38

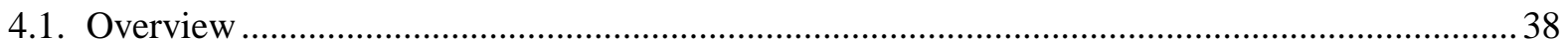

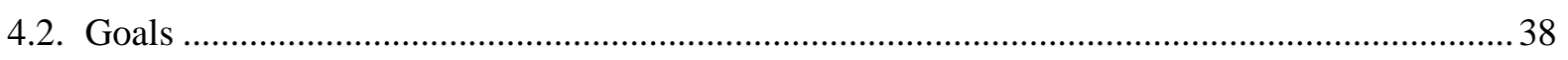

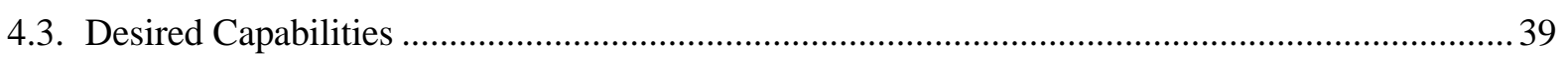

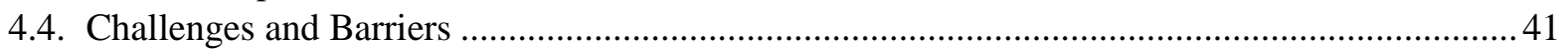

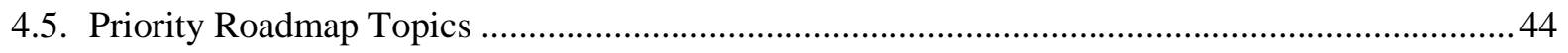

5. Crosscutting Challenges ............................................................................................. 49

6. Next Steps .............................................................................................................................. 51

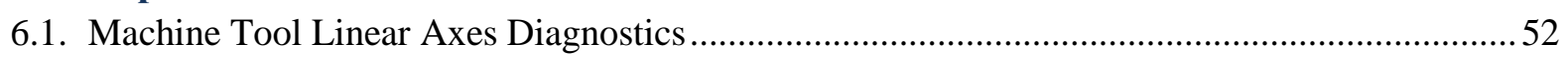

6.2. Systems-Level Monitoring, Diagnostics, and Prognostics .....................................................5

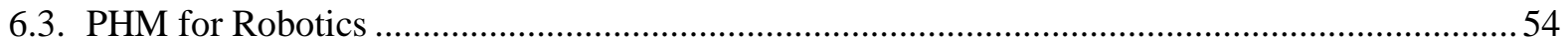

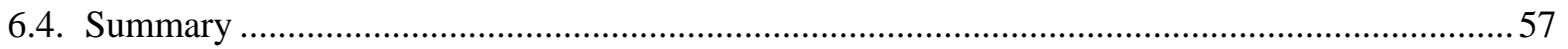




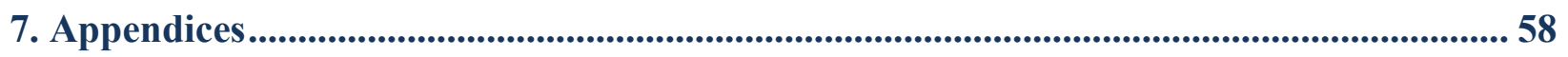

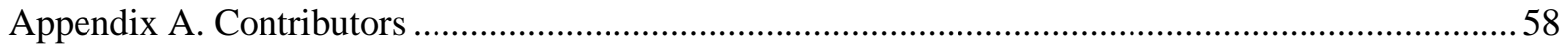

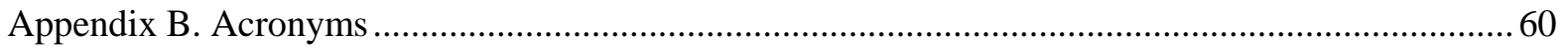




\section{Introduction}

\subsection{Overview of PHM}

Prognostics and Health Management (PHM) technologies are vital in today's manufacturing environment. PHM can reduce the time and costs for maintenance of equipment or manufacturing processes, and minimize equipment/process fault and failure potentials, through the application of efficient, costeffective condition monitoring, diagnostic, prognostic, and maintenance tools. Another key benefit of PHM is that it can aid the optimization of overall equipment effectiveness (OEE); i.e., the ideal (yet virtually impossible) scenario of operating $100 \%$ of the time at $100 \%$ capacity, with an output of $100 \%$ good quality. Improved OEE means fewer equipment failures and reductions in stoppages, waste, rework, and start-up losses. Overall, the objective of PHM is to provide timely actionable information to enable intelligent decision - making for improved performance, safety, reliability, and maintainability.

\subsection{Background and Importance of Prognostics and Health Management}

Information and communication technologies are converging with many advanced and emerging physical technologies (including sensing, automation, machining, robotics, and additive manufacturing) to drive profound transformations to manufacturing, creating the next generation of industry. These Smart Manufacturing Systems (SMS) are paving the way for more efficient, automated, programmable, and flexible forms of manufacturing to meet changing consumer demands (Huizinga, 2014). The competitive advantage gained through the implementation of SMS has cross-cutting benefits to multiple industries. New studies show that for every one job created in a Smart Manufacturing plant, two, three, four or more "indirect" jobs are created outside the plant in companies to service, support and supply it (Bernaden, 2012). The importance of continued innovation in manufacturing, and especially advancing smart manufacturing systems, has been noted in numerous significant reports over the last several years, spurring continued investment and research (McKinsey, 2012; PCAST, 2011; PCAST, 2012; PCAST, 2014). As these reports note, advanced robotics, new information technologies, and sophisticated telecommunications capabilities are critical technological innovation areas for advancing manufacturing. The availability of large amounts of data ('big data') has also been cited as key to driving advances in smart manufacturing, provided data can be turned into actionable insights or new operating improvements.

The potential of smart manufacturing to revitalize U.S. manufacturing is driving new research investments and initiatives. For example, a Digital Manufacturing and Design Innovation Institute (DMDII) has been launched as one of the innovation hubs in the new National Network for Manufacturing Innovation. ${ }^{1}$ The DMDII sponsors research projects in digital manufacturing and design; disseminates the lessons learned; and helps educate tomorrow's manufacturing workforce. ${ }^{2}$

\footnotetext{
${ }^{1}$ www.manufacturing.gov

2 http://dmdii.uilabs.org/
} 
Investments in SMS across the globe are also taking advantage of these growth opportunities. For example, the Factories of the Future (FoF) Public-Private Partnership aims at helping EU (European Union) manufacturing enterprises and amounts to $€ 1.15$ billion over a seven-year period from 2014-2020 (European Commission, 2013). Maintaining a domestic technical edge over the global competition will require focused actions and broad support.

The development of SMS is dramatically increasing the complexity of system, sub-system, and component interactions within manufacturing processes. It is challenging to determine if/when specific failures will occur, how the impact can be minimized, and how failures can be mitigated in the future. Prognostics and health management (PHM) combines sensing and interpretation of environmental, operational, and performance-related parameters to assess equipment health, predict remaining useful life (RUL), and diagnose failures (should they occur). In some cases, sensor readings are fed into one or more control systems that monitor potential fault and failure indicators such as vibration levels, flow rates, temperatures, chemical concentrations, and strains to provide real-time reporting to the equipment operators (Pecht, 2008). A potentially more effective approach is using PHM modules or data collectors to collect and analyze sensor data and other health information

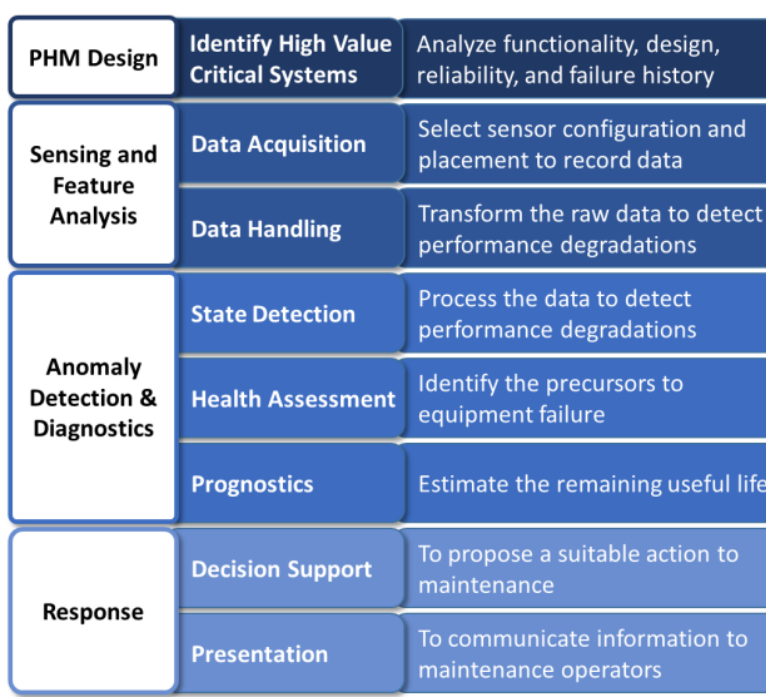

Figure 1-1. General Prognostics and Health Management Design Components to Detect and Mitigate Equipment Failure decoupled from the process or equipment's control system. A separate PHM system offers more flexibility to add sensors, software, without the need to alter the control system, which can be difficult and expensive to change and re-qualify (Mooney, 2015). The basic components of a PHM system are depicted in Figure 1-1 (adapted from Pecht, 2008).

PHM for SMS can enable more efficient maintenance strategies such as Total Predictive Maintenance $(\mathrm{TPM})^{3}$. These strategies engage all levels and functions in an organization to maximize the effectiveness of production equipment. PHM for smart manufacturing will facilitate smarter, safer, and more environmentally sustainable manufacturing processes. Estimates from the U.S. Environmental Protection Agency (EPA), which look at manufacturing efficiency as a means of improving environmental performance, show companies can realize a $15 \%$ to $25 \%$ increase in equipment efficiency rates within three years of adopting TPM strategies (EPA, 2011).

As shown in Figure 1-24, the total maintenance cost is impacted by the cost of repairs, the cost of proactive maintenance (e.g., PHM system installation and operation to proactively address equipment failures before they occur), and the cost of production losses and downtime. Good proactive maintenance programs can improve uptime, increase longevity, and improve maintenance cost control and operational safety. With the growing use of smart systems and networks in manufacturing, more equipment and process data is available for proactive asset management. Structured data obtained from equipment and

\footnotetext{
${ }^{3}$ Jay Lee and Edzel Lapira, "Smart: PHM algorithms help Total Predictive Maintenance step up its game.” Intelligent Maintenance Systems Center, University of Cincinnati, June 2011. http://www.imscenter.net/IMS_news/2011-tpm-gets-smartims.pdf

${ }^{4}$ Life Cycle Engineering. Accessed 12/27/14. http://www.lce.com/pdfs/The-PMPdM-Program-124.pdf,
} 
assets can be combined with more sophisticated analytics to create opportunities for better reliability, uptime, and longevity.

By detecting changes in performance, PHM can alert operators to interventions needed to maintain process optimization, subsequently improving OEE. The improved understanding of OEE made possible through PHM enables the plant to get the most out of process systems and can have a large impact on the cost-benefit model for PHM. As a result of OEE improvements, for example, PHM systems could be justified without considering cost reductions and avoidances (e.g., secondary damage).

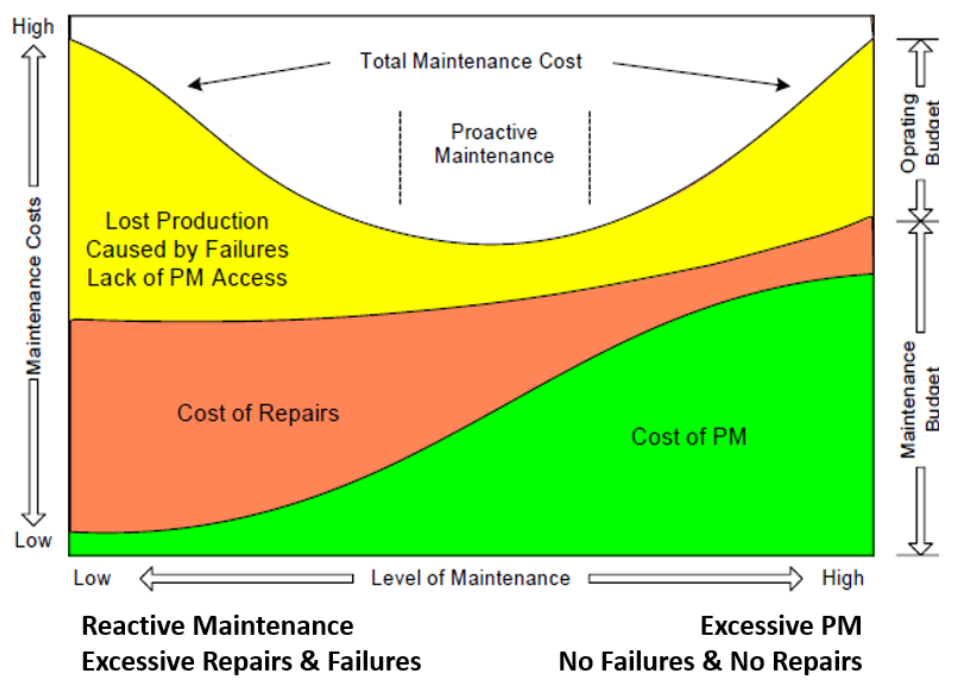

Figure 1-2. The Maintenance Cost Paradigm: Total maintenance related cost $=$ Cost of Proactive Maintenance + Cost of Repairs + Cost of Production Losses caused by Failures \& PM Time (LCE 2014).
For proactive maintenance to be effective, the potential failure modes of the equipment must be identified and precursors to failure monitored.

However, as equipment, processes and operating environments evolve, failure modes and physics can change. PHM systems need to be open and adaptive to changes in all these areas. Complex, software intensive equipment and processes also do not lend well to typical physics of failure models. Developing effective proactive systems and models requires a solid understanding of the physics of failure and how these may change as the environment and equipment changes. Applying that level of understanding into predictive algorithms and analytics, and incorporating it into training, can help operating personnel interpret pre-failure conditions and take corrective action. Analytics are also a key component of PHM. In some cases, analytics can root out complex behavior patterns better than physical models (Mooney, 2015).

As smart manufacturing expands in the United States, further design, verification, validation, implementation, and analysis of PHM systems will be needed. Measurement science plays an important role and can be used to enhance condition monitoring, diagnostics, and prognostics at many levels within a factory. For example, improvements in data management and analysis will play a key role in identifying signals among noise, reducing the occurrence of false positives. Additionally, better software will enable real-time, online equipment monitoring to provide more timely actionable intelligence. Measurement science will facilitate diagnostic- and prognostic-related information sharing among levels within a factory to minimize downtime, operating and maintenance $(\mathrm{O} \& M)$ costs, and energy requirements.

Standards will provide guidelines for conducting development, testing, and implementation of PHM technology. Standards cover such aspects of the field as: terminology, definitions, methodologies, metrics, specifications, testing, software (SW), hardware (HW), monitoring, analysis, modeling, products, systems, reliability, safety, ratings, application, best practices, compliance, management, and training. The standards developed and approved through consensus will be the guidelines by which organizations 
can proceed with PHM implementation. Recent studies have been published that review a number of the key standards relevant to PHM (Vogl et al., 2014a; Vogl et al., 2014b).

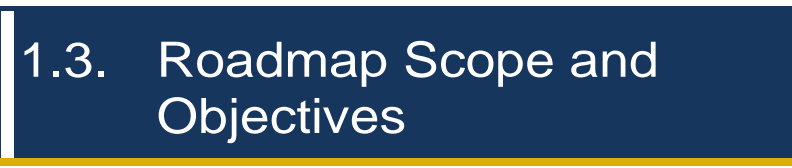

The National Institute of Standards and Technology (NIST) hosted the Roadmapping Workshop: Measurement Science for Prognostics and Health Management of Smart Manufacturing Systems (PHM4SMS) at its Gaithersburg, MD campus on November 19-20, 2014. The event brought together over 60 PHM experts from industry, government, national laboratories, and academia to identify measurement science challenges and associated research and development $(\mathrm{R} \& \mathrm{D})$ needs for PHM in the context of smart manufacturing. The objectives of the workshop were to:

- Serve as a key building block for the creation of a measurement science roadmap for PHM4SMS research, by developing information on:

- Measurement science barriers, challenges, and gaps preventing broad use of PHM4SMS

- Research and development (R\&D) needed to address the priority measurement and standards challenges

- Future measurement- and standardsrelated targets and goals for PHM

- Actions to overcome the high priority barriers

- Inform future NIST technical programs and strategic planning

- Offer valuable information to other government agencies and stakeholders focused on PHM within manufacturing environments

\section{NIST Smart Manufacturing Operations Planning and Control Program}

At the National Institute of Standards and Technology, the Smart Manufacturing Operations Planning and Control Program (SMOPAC) is conducting R\&D on PHM systems to facilitate the adoption of smart manufacturing (fullyintegrated, collaborative manufacturing systems that respond in real-time to meet changing demands and conditions in the factory, in the supply network, and in customer needs). The overall program will aid in development of efficient networked sensing and control, prognostics and health management (including diagnostics and maintenance), integrated wireless platforms, industrial control security, efficient information exchange and interoperability of system components. Testbeds will be used to evaluate architectures, standards, and scale effects for reference implementations. The resulting manufacturing tools and resources will enable: increased efficiency of operations; manufacturing process development cycles at reduced risk, time and cost; and orders of magnitude expansion of manufacturing operations across increasingly diverse market segments. Additional information about the SMOPAC Program can be found at http://www.nist.gov/el/msid/syseng/smopc.cfm

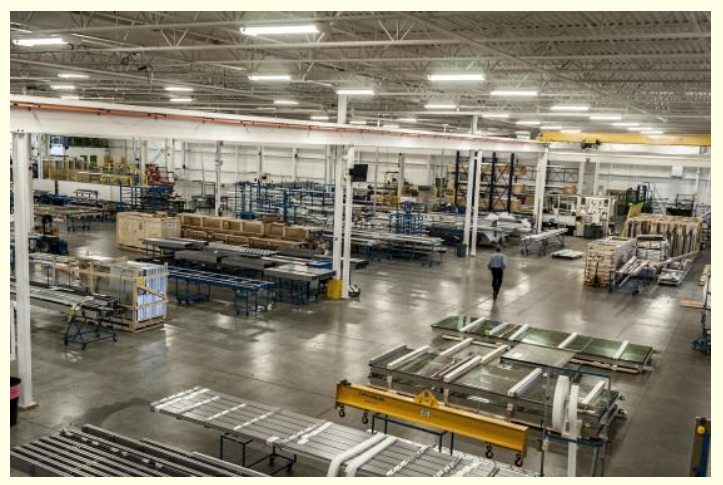

Photo Credit: DPC 64341250

The Roadmap for Measurement Science for Prognostics and Health Management of Smart Manufacturing Systems is based largely on workshop discussions as well as insights provided by speakers and panelists. The report is organized around three significant topic areas: 
- PHM Manufacturing Process Techniques and Metrics - current and projected PHM techniques and methods for application to smart manufacturing systems; metrics used to monitor system health and enable PHM techniques; and the relationships between techniques and metrics.

- PHM Performance Assessment - current and future approaches to assess the performance of PHM systems including (but not limited to) validation and verification of methods and uncertainty quantification.

- PHM Infrastructure: Hardware, Software, and System Integration - current and future hardware and software technologies (including sensors, controllers, models, and simulations) to enable or integrate with PHM techniques.

The ideas presented here are a reflection of the attendees and not necessarily the entire industry. As such, they should be viewed as a representative sampling of the important perspectives, but not all-inclusive. The participants were selected based on their high level of technical knowledge related to PHM technologies, systems, and practices and are considered experts in the field.

This report details the findings from the first-ever event to focus on identifying the most pressing measurement science challenges and associated research and development (R\&D) needs for PHM4SMS. The information is useful to both public and private decision-makers interested in furthering the capabilities of PHM and expanding its use in the smart manufacturing sector. It is envisioned that the national research agenda for PHM will incorporate some of the needs and challenges detailed in this report. Manufacturing and engineering personnel (plant managers, machine operators, reliability engineers, technology integrators, etc.) involved in making process improvements will also find this report useful.

\subsubsection{Workshop Process}

Several key questions and focus areas were posed to gain insights on the important challenges and pathways to address them.

\section{Desired Capabilities}

- Envisioned future: What capabilities are wanted and needed the most?

\section{Challenges and Barriers for Achieving the Capabilities}

- What technologies or other barriers limit development, implementation, and/or integration of PHM?

- What are the measurement and standards barriers, challenges, and gaps?

\section{Priorities}

- What are the Research and Development $(\mathrm{R} \& \mathrm{D})$, and standardization priorities?

Pathways for Measurement Science Roadmap

- Identification of the R\&D activities, standards, and approaches for addressing priority challenges

- Next steps and actionable plan

\subsubsection{Prioritization Process}

Each group used a real-time voting scheme ( 5 votes per person, same value for each vote) to indicate which challenges were of the greatest priority to address. When voting, participants were asked to 
consider a set of criteria, including 1) the challenges most urgent (timing) to address that would accelerate the development and use of PHM for smart manufacturing; and 2) challenges that would have the most impact on parameters important to manufacturing (i.e., productivity, equipment/process performance, cost effectiveness, and quality). After prioritizing the challenges as high, medium, or low, several of the higher priority challenges were examined more closely to create a roadmap for R\&D, standards development, and other future efforts for PHM4SMS.

\subsection{Plenary and Panel Summaries}

Presentations from leading industry experts set the stage for workshop discussions. As permitted by the authors, presentations can be downloaded at http://www.nist.gov/el/isd/phm4sms-workshop.cfm. The presentations of speakers and panelists reflect unique opinions from their respective fields. Many of the themes identified were echoed during the breakout sessions and further expanded upon.

\section{Plenary Talks}

\section{- Day 1 - Opening Remarks:}

\section{Howard Harary, Director, Engineering Laboratory (EL), NIST}

Welcome and purpose: NIST welcomes you to provide your expert inputs on the challenges and needs for prognostics and health management for smart manufacturing systems. The NIST Engineering Laboratory (EL) is currently pursuing work in PHM in a number of areas, and your insights will help to guide our future efforts.

- Workshop Scope and Objectives:

Brian A. Weiss, Project LeaderPHM4SMS, NIST

\section{Synopsis of workshop scope, process,} and desired outcomes: NIST has a unique mission; measurement science plays an important role in the context of creating critical-solution enabling tools - including metrics, models, test methods, and knowledge - for U.S. manufacturers. Priorities and next steps need to be identified to address key measurement science needs, challenges, and gaps that are hindering the development and deployment of health monitoring, diagnostics, and prognostics technologies at multiple levels within a factory.

\section{Successful Development and Implementation} of PHM Systems: The Center for Intelligent Maintenance Systems (or IMS)

The Industry University Cooperative Research Center Program of the National Science Foundation (IUCRC/ NSF) has supported the development of the IMS since 2001.

The Center collaborates with over 30 research institutions and over 70 industry partners worldwide - including Boeing, Ford, Toyota, and United Technologies. These companies validate, deploy, and benefit from PHM technologies such as embedded and remote monitoring, prognostics technologies, and intelligent decision support tools ${ }^{3}$.

Every dollar invested in the IMS exhibits a benefit to cost ratio of 238:1 in return. Investments between 2001 and 2010 resulted in a returned value of more than $\$ 855 \mathrm{M}$ created by IMS knowledge and technology (Gray 2012).

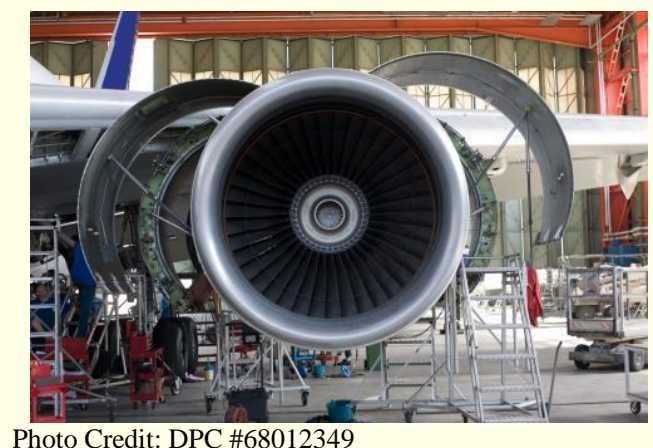


- Health Management of Smart Manufacturing Systems: Al Salour, Senior Technical Fellow, The Boeing Company

Boeing experience with implementing prognostics and health management systems into their manufacturing processes: Common standards and interoperability between systems will benefit software-assisted diagnosis and prognosis. This is a challenge in a large organization because business units have their own preferences and have even created unique software. One of the main issues currently faced is the amount of time it takes to direct data towards a decision. A lot of data is collected, yet current technology doesn't provide enough actionable information. Emerging tools and advancements are improving software-assisted diagnosis and prognosis to provide more actionable information and facilitate quicker decision-making.

\section{- Day 2 - Opening Remarks}

Albert Wavering, Chief, Intelligent Systems Division (ISD), EL, NIST

Advances in intelligent manufacturing: The Intelligent Systems Division develops and advances measurement science and standards to speed development, adoption, and integration of leading-edge intelligent technologies that will improve U.S. manufacturing performance. A key aspect of ISD's work is enabling real-time monitoring, control, and performance optimization of smart manufacturing. The Internet of Things combined with cyber-physical systems (highly networked information technology/IT and physical systems) has the potential to change the face of manufacturing. As the use of intelligent systems grows in this sector, new technologies and strategies for prognostics and health management will be needed to keep pace and to take advantage of new opportunities. This workshop is a good forum for gaining insights on how PHM can adapt and advance in new smart manufacturing environments.

\section{- Recent Advances and Transformation Direction of PHM:}

Jay Lee, Professor at the University of Cincinnati, Founding Director of National Science Foundation (NSF) Industry/ University Cooperative Research Center (I/UCRC) on Intelligent Maintenance Systems

Evolution of advanced manufacturing facilities, product lifecycle management, and the emergence of PHM: Despite improvements to PHM systems, the goal of achieving near-zero breakdown has still not been achieved. In the next 10 years, PHM systems need enhanced control and self-maintenance systems that are robust in the manufacturing environments of the future. In advancing the deployment of PHM systems, it is crucial to learn from past mistakes and plan what can be done in the future to make systems more valuable. Data collection, interpretation, and storage will play an important role in the improvement of these systems. 


\section{Panel 1: PHM Capabilities, Best Practices, Challenges, and Needs}

This panel session reviewed the current state of PHM related to application in manufacturing environments. Panelists provided insights on PHM technology and systems, in particular current capabilities, best practices, and challenges, as well as technology gaps and limitations. The moderator and panelists are listed in Figure 1-3; key points are summarized below.

- Andrew Inman, Toyota

Reliability and Maintenance

Toyota initiatives around reliability and maintenance and how to accelerate deployment of PHM within Toyota manufacturing plants: Machine maintenance is essential to maintaining efficient production of quality vehicle products that create consumer confidence. PHM efforts include the design of self-diagnosing machines and the use of effective process analysis to prevent failures and their reoccurrence. All of the systems have to work together to be effective; this is a challenge due to the differences in the systems and the vast amounts of data collected. Because manufacturing groups often work in silos, it is difficult to connect data and provide feedback to various groups. Implementing a computerized maintenance management system (CMMS) may improve connectivity between the different groups and increase reliability.

- Carl Byington, Impact Technologies/ Sikorsky/ United Technologies

Best Practices in PHM and Application to Manufacturing

Hardware and software products being developed by Impact Technologies and how they are being integrated into Sikorsky helicopters and manufacturing processes: Safety and overall equipment effectiveness (OEE) are essential components of the Impact approach to Total Productive Maintenance (TPM). OEE takes into account all losses (down time, speed, and quality), resulting in a measure of truly productive manufacturing time. The value of prognostics is information and how it is used to improve production and reliability. One of the key concepts is moving away from reliability-centered maintenance towards condition-based maintenance + prognostics $(\mathrm{CBM}+)$. This approach involves utilizing the assessed health or diagnostic fault classifier output to predict a failure evolution. Typical manufacturing environments have rich data potential to develop greater prognostics.

- William Marsher, Mechanical Solutions Inc. Manufacturing Machinery PHM

Greatest challenges, needs, and opportunities for manufacturing PHM: Data is at the core of successful PHM. However, data collection and analysis is difficult to automate and can lead to false positives. Industry systems still require a human in the loop to analyze the data and verify problems. Developing better software to provide on-line monitoring with actionable information available in real-time would be difficult, but well worth it. Statistical data provides a good starting point, but machine/physics-based algorithms are necessary to put the information into a form that makes sense. With modern hardware, this software and information can be managed with a great degree of success to maintain control and reduce downtime.

- David Siegel, Predictronics Corporation Manufacturing PHM Successes and Challenges 
Overview of PHM manufacturing applications, past PHM successes in manufacturing, and the key challenges to PHM implementation: Obtaining the right data is essential to effective diagnostics and prognostics. PHM at the machine level is complicated by the multitude of operational settings. Normalizing this data to readily distinguish between routine operation and a problem is difficult. Additionally, data obtained from multiple places is often not uniform and maintenance records may be incomplete or unstructured. These inaccuracies and inconsistencies further complicate the use of historical data to develop and validate diagnostic and prognostic systems.

\section{- Highlights of Q\&A}

Human error and scarcity/cost of experts: Self-diagnosing machines are needed to prevent failure through process analysis and prevent reoccurrence. Implementing more physics into PHM software will reduce the need for experts to predict how a machine will behave, result in more consistent prognostics, and expedite the process. Expert tribal knowledge can be better leveraged in the design of PHM technologies which would significantly reduce the need and cost of human expertise during equipment monitoring and diagnostics.

Systems communication and interoperability: Multiple systems, legacy equipment, and nonuniform software complicate PHM systems. Improving connections between systems; and managing and interpreting the vast amounts of data collected are essential to PHM development.

Multiple data sources for understanding failure: It is difficult to obtain a sufficient training set of data to determine when equipment failure will occur. In many cases, letting enough systems fail to be useful for statistics is not an option. There are opportunistic failure events where we can learn from failure but testing and modeling are important to deciphering and validating failure data.

Real-time aspects of PHM systems: The need for real-time PHM systems represents a significant development challenge. When there is a high value asset important to production, it is useful to have real-time PHM to minimize fault and failure occurrences; and provide peace of mind on equipment functionality. Some organizations aren't ready for (or need) that level of speed and can instead focus on other systems.

Key performance indicators (KPIs) at the component and system level: Most componentlevel KPIs are based on technical performance. The next level is effectiveness metrics and how that manifests at a system level (e.g., maintenance hours/ operation hours), algorithm level, and OEE at production level. There is a need to determine the appropriate KPIs to support PHM.

Security of PHM systems: This is a big concern, especially for the military. Information assurance is an evolving concept, and a hurdle for those developing systems. Refineries and power plants are reluctant to implement wireless technologies and are afraid of hacking. Wireless technologies can make PHM capabilities more convenient. Convenience needs to be balanced with developing a secure distributed architecture to mitigate cybersecurity concerns. 


\section{Panel 2: Performance Assessment - Monitoring and Measurement}

This panel focused on techniques for measuring and/or monitoring how well PHM systems are performing their functions in smart manufacturing environments, as well as metrics that can be used to measure overall manufacturing performance (to isolate the impact of PHM technologies on manufacturing performance). The moderator and panelists are listed in Figure 1-4. The key points that emerged are summarized below.
Figure 1-4. Performance Assessment Monitoring and Measurement

Moderator, Moneer Helu, NIST

- Harry Kekedjian, Ford Motor Company

- Kai Goebel, NASA

- William Sobel, System Insights

- John Oskin, Sage Clarity

- Harry Kekedjian, Ford Motor Company

Predictive Maintenance Strategy

Use of Predictive Maintenance (PdM) to assess the condition of in-service equipment and determine when maintenance should be performed: In theory, PdM provides cost savings over routine preventative maintenance, because maintenance tasks are performed only when warranted. In practice, limited plant resources and budgets make it challenging to perform maintenance tasks in a timely manner; and data collection and processing has been very monotonous and labor intensive. False positives are a common occurrence that need to be reduced. Information needs to be clean and clear, even when complex algorithms are involved, to enable rapid decision-making. PdM plans must make efficient use of limited resources and provide accurate, fast results to be effective.

\section{- Kai Goebel, NASA}

\section{Performance Assessment - Monitoring and Measurement}

Performance assessments for prognostics systems: An effective prognostic algorithm for performance assessment requires multiple components. Important factors, such as minimizing downtime, must be embedded at an algorithmic level. A cost-benefit analysis and failure mode, effects, and criticality analysis (FMECA) will justify the investment of developing a prognostic system, including the identification of necessary sensors. Once in place, the prognostic system can be calibrated to characterize normal operations and fault modes. Prognostics metrics can then be used to evaluate, refine, and improve the ability of the PHM system to measure performance (e.g., accurate and timely algorithm predictions for equipment end of life parameters).

\section{- William Sobel, System Insights}

\section{Vimana, Predictive Analytics Platform for Manufacturing Intelligence}

Development of predictive analytics for manufacturing: Most manufacturing processes are run sub-optimally from a tool and performance perspective. Now that improved sensors and technologies are becoming available, real-time data analytics, downtime and process improvement, and prognostics that go beyond machine health are possible. Presently, there are no common standards for the level(s) in which diagnostic and prognostic activities need to occur on onboard equipment vs. in the cloud. Flex computing at System Insights (commonly referred to as fog computing), takes an integrated approach by performing big data analytics in the cloud, in addition to shop floor processing, to find optimal process parameters for use in production. 


\section{- John Oskin, Sage Clarity}

One View Methodology, Move from Static to Real-time KPIs

Moving from static to real-time prognostics metrics: Making a move from static to real-time KPIs can be costly in terms of both time and money. "Real-time" is a relative term, where timeliness could be monthly or hourly metrics, for example. KPIs for manufacturing performance, quality, safety, cost, and work order compliance need to be sorted according to factors such as their complexity, accessibility, and business priority. The transition often must occur as a multi-year strategy with an established roadmap to address the prioritized KPIs.

\section{- Highlights of Q\&A}

Encourage suppliers to monitor equipment and capture data: It is difficult to push down PHM to the equipment of suppliers. The suppliers that implement PHM techniques will have a competitive advantage. Clarity is crucial when communicating PHM requirements to suppliers. If a requirement is vague, vendors will often interpret it as a minimum.

Justify the cost benefit of PHM systems: When looking at equipment that does not fail often, the cost benefit needs to be tied back to quality and safety- a healthy machine builds healthy parts. PHM system developers can also participate in warranty guarantees of their services to increase manufacturer confidence in PHM (e.g., any unpredicted maintenance is free).

\section{Panel 3: PHM and the Human Element}

This panel focused on the impact and interpretation of human decisions on PHM in smart manufacturing environments, and challenges to be faced when humans interact with PHM technologies. The moderator and panelists are listed in Figure 1-5. The key points that emerged are summarized below.
Figure 1-5. PHM and the Human Element

Moderator, Patrick Brown, University of Cincinnati

- Thomas Mooney, SOAR Engineering LLC

- Andrew Hess, The Hess PHM Group

\section{- Thomas Mooney, SOAR Engineering LLC}

Integration of PHM into business operation systems: Traditional PHM systems are too often isolated, stand-alone systems based on simple cause-and-effect models. PHM is not just a technical system where the human interaction and user experience pieces can be trivialized. The PHM environment needs to be flexible to accommodate the inclusion of various people, organizations, training, and leadership. There are plenty of sensors and data capable of providing actionable information. Systems can be integrated to create value for all participants in the value chain and enable evidence-based decision making. The best PHM systems are not fully human or fully autonomous, but a hybrid where the machine intelligence augments the human as an ally, and not an adversary. It is important that PHM systems learn, adapt, influence (e.g., decisionmakers), and communicate across the organization.

\section{- Andy Hess, The Hess PHM Group}

Better asset management through improved information on actual equipment condition: Predictive prognostics, along with condition management, provide critical information, leading to a significantly more effective and beneficial integrated asset management system. Factors such as warranty guarantee tracking, prognostics, logistics, and CBM+ management play an important role in and are key enablers for new business practices and decisions. All industry sectors warrant some PHM and stand to benefit at various levels. For example, a $1 \%$ improvement in corrosion monitoring through PHM could save billions of dollars worldwide. Operator buy-in is needed to effectively develop, apply, and manage these systems to yield the greatest impact. 


\section{- Highlights of Q\&A}

Improve understanding of refurbished equipment: It is difficult to estimate the remaining life of refurbished equipment. Replacing parts within a larger system complicates the ability to predict when the system may fail. PHM with inventory tracking could note the differences in remaining useful life between individual parts - but this requires extensive record keeping.

There are broad applications for PHM systems that decision-makers will support: Target systems for PHM are often the most expensive and critical components of the manufacturing process, for example where costly process disruptions or creation of unsafe working conditions and liabilities are vital to avoid. However, some products or processes contain non-expensive components for which their failure could lead to devastating consequences (i.e., lithium-ion batteries on an airplane) - PHM has important applications in this domain, as well.

PHM is best implemented and sold during the installation of the manufacturing process: It's always cheaper to implement a PHM system in the beginning (e.g., when starting a new design/process) rather than after the fact. Determining the level of performance required for the PHM system in the beginning can help to determine how varying levels of maintenance and downtime will impact production and profitability. Tying reduced maintenance costs and productivity increases to PHM can help demonstrate the value of implementing a PHM system from the start.

The human element of buy-in and support for PHM should not be overlooked: The motivation to utilize a PHM system must come from upper management, whom are more likely to endure the efforts to implement a long-term savings strategy than middle management. Once implemented, experience indicates that local operators and others within the organization will realize that the data gives them great feedback and they quickly learn how to operate the system.

It is becoming more difficult for humans to validate software: As software code becomes more complex, it is becoming harder to validate and humans are becoming less involved in this validation process. It is also difficult to verify and validate systems that include people because the systems are dynamic and subject to change. Additionally, the longer a complex code is used, the more likely it is to generate/produce errors. PHM is needed for software to maintain a system's flexibility to accommodate changing conditions.

\subsection{References}

Bernaden, John., 2012. Indirect Jobs: A direct way to talk about why we need smart manufacturing. Rockwell Automation. http://smartmanufacturing.com/wp-content/uploads/2012/01/SmartManufacturing-increases-indirect-jobs.final_.pdf

EPA, 2011. Lean Thinking and Methods, Total Productive Maintenance. Environmental Protection Agency. Accessed January 3, 2015. http://www.epa.gov/lean/environment/methods/tpm.htm

European Commission, 2013. Factories of the Future PPP: Towards competitive EU manufacturing. http://ec.europa.eu/research/press/2013/pdf/ppp/fof_factsheet.pdf

Gray, D.O. et al., 2012. Measuring the Economic Impacts of the NSF Industry/ University Cooperative Research Centers Program: A Feasibility Study. Prepared for National Science Foundation (NSF) by North Carolina State University. Final Report.

Huizinga, G. Walison, P. van der Beek, H. Tops, P. Wijnstok, J. Heemskerk, P. van Os, M. van der Horst, T. Helmer, S. Huveneers, S. Butter, M. van der Zee, F. van Oort, S. Ypma, J. Mulder, G. 
Kotternink, B. de Heide, M. Grosfeld, T., 2014. Smart Industry, Dutch Industry Fit for the Future. http://www.smartindustry.nl/wp-content/uploads/2014/07/Opmaak-Smart-Industry.pdf

McKinsey 2012. Manufacturing the future: The next era of global growth and innovation. McKinsey Global Institute, Global Operations Practice. November 2012. http://www.mckinsey.com/insights/manufacturing/the_future_of_manufacturing

Mooney, T., 2015. Personal Communication. Soar Engineering, LLC.

Pecht, M., 2008. Prognostics and Health Management of Electronics; Wiley-Interscience: New York, NY, USA.

PCAST 2011. Report to The President on Ensuring American Leadership in Advanced Manufacturing. Executive Office of the President, President's Council of Advisors on Science and Technology (PCAST). June 2011.

PCAST 2012. Report to the President on Capturing Domestic Competitive Advantage in Advanced Manufacturing. Executive Office of the President, PCAST. July 2012.

PCAST 2014. Report to The President: Accelerating U.S. Advanced Manufacturing. Executive Office of the President, PCAST. October 2014.

Vogl, Gregory W., Weiss, Brian A., Donmez, M A., 2014a. "Standards for Prognostics and Health Management Techniques within Manufacturing Operations." Annual Conference of the PHM Society, September 29-October 2, 2014. http://www.nist.gov/customcf/get_pdf.cfm?pub_id=916376

Vogl, Gregory W., Weiss, Brian A., Donmez, M A., 2014b. "Standards Related to Prognostics and Health Management (PHM) for Manufacturing." NIST Interagency/Internal Report (NISTIR), July 7, 2014. http://www.nist.gov/manuscript-publication-search.cfm?pub id=915550 


\section{PHM Manufacturing Process Techniques and Metrics}

\subsection{Overview}

Section 2 presents the Roadmapping Workshop results of a breakout group focused on the topic of PHM Manufacturing Process Techniques and Metrics. The section summarizes the discussions and activities performed by this breakout group. Participants identified goals, desired capabilities, challenges and barriers, and priority roadmap topics. A list of participants from all breakout groups is shown in Appendix A.

PHM techniques can have a dramatic impact on manufacturing operations by enabling pre-fault/prefailure maintenance actions based on need rather than the use of schedules (e.g., where maintenance is performed by time intervals irrespective of need). PHM enables a determination of the current system condition via diagnostic analyses, and provides insight into expected future conditions through prognostic methods.

PHM manufacturing process techniques and metrics encompasses a wide spectrum of process and equipment condition monitoring technologies and systems; the factors influencing conditions; issues affecting equipment criticality (e.g., cost of machine downtime, replacement costs); relevant condition monitoring parameters (such as temperature, pressure, and vibration) for various machine types; and correlation of possible faults and failures. PHM also supports smart manufacturing, where systems and equipment are highly-interconnected, by integrating maintenance operations via data collection, diagnostics, prognostics, and usage monitoring.

In a smart manufacturing environment, PHM process techniques must consider entire systems rather than individual components. This adds to the complexity of PHM and necessitates advances in standards, data collection and analysis techniques, data management, system training, and software interoperability. As information and communications technologies become more integrated into manufacturing environments, the need for advanced PHM, including supporting standards

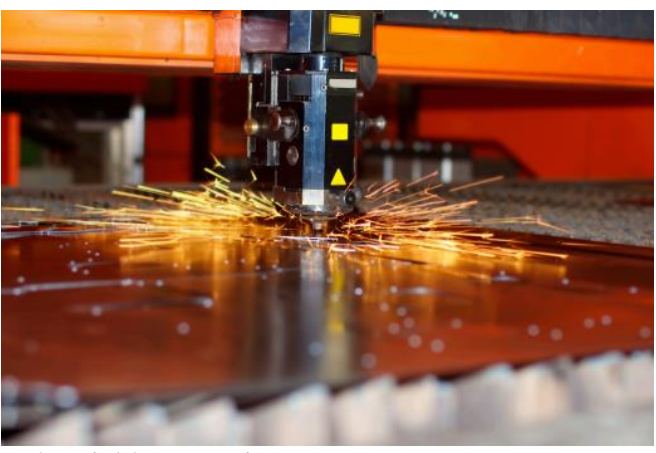

Industrial laser cutting process Photo Credit: DPC \#65898405 and data management, will become increasingly important.

\subsection{Goals}

The goals identified for PHM manufacturing process techniques and metrics emphasize condition monitoring, substantial improvements in reliability (reduction in non-scheduled breakdowns), improved 
safety, operator situational awareness, and overall equipment effectiveness (OEE). Table 2.1 provides highlights of the goals identified, organized by major emphasis category.

The objective would be to enable PHM techniques that are capable of evolving appropriately as new manufacturing equipment or processes are introduced. PHM systems should ultimately support enhanced product quality via improved manufacturing performance, i.e., PHM can be used to enhance product and process resiliency over the life cycle. Knowledge of what is failing or negatively impacting KPIs is essential to learn what can be translated into product and process improvements for existing or future systems. PHM system evolution can also lead to varying degrees of autonomy, enabling systems to selfmonitor and heal.

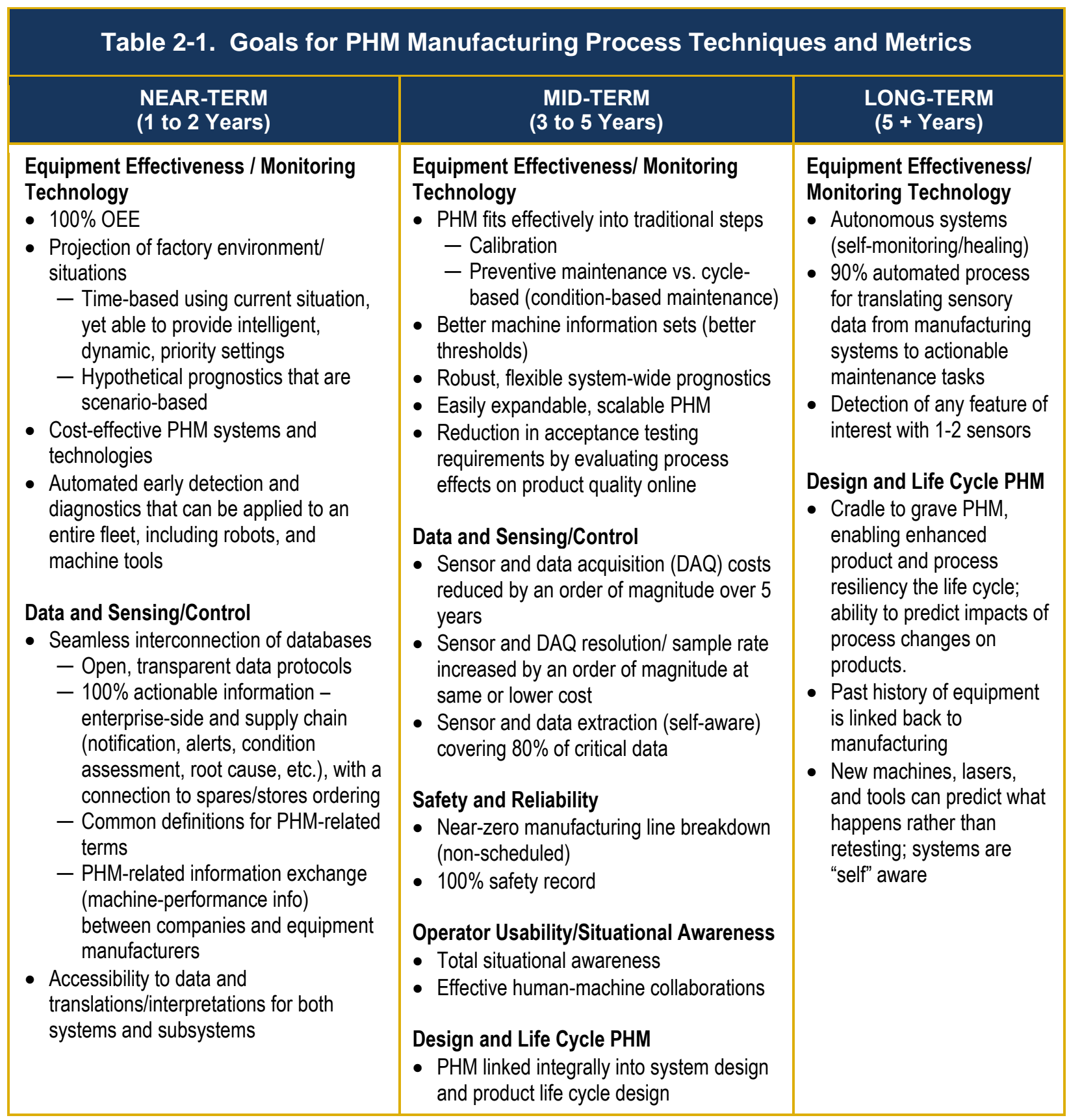




\subsection{Desired Capabilities}

A number of key capabilities were identified as important to support PHM for smart manufacturing processes; these are outlined in Table 2.2 and summarized below. Priority for capabilities was determined using a real-time voting scheme, described in Section 1.3.1, Workshop Process.

\section{Robust PHM Systems}

Robust PHM systems are needed to support increasingly complex and interconnected smart manufacturing systems. These PHM systems would ideally be reconfigurable, scalable, have robust prognostics and diagnostics, operate in real-time, and be accessible on-line. They would include dynamically-reconfigurable tools with the capability for scenario-recognition and awareness to predict conditions and situational awareness.

Agility is also a key element for robust PHM systems. This would include flexibility in configuration; sensing and data analytics; and the ability to rapidly adjust to new hardware and software as the manufacturing process evolves. Distributed PHM concepts and standards need to be developed to support agility and flexibility. Smart sensing capabilities will be needed for machine collaboration related to PHM.

\section{Data and Sensing/Controls}

A high priority is capabilities that allow for the effective, secure collection, management, and translation of data into actionable information. Universal interfaces for machine data (e.g., machine readable), communication protocols, and data standards are key aspects. Standards are needed for the common measurement and classification of data. Data acquisition and storage capabilities are required at a sufficiently high resolution, while preserving the meaning of data. Semantic recognition of data and links between records should be coupled with relational knowledge preservation. Integrated networks and interoperability will be required to enable "seamless data flow" of communications for PHM. Such systems should also be efficient (e.g., only handle data once). Guidelines are needed to identify what specific data is important to extract the most value from all types of data and prioritize data. While large volumes of data are possible, not all of the data is critical to decision-making. Better "Big Data" distributed query and analytical tools are needed to enable plant-wide PHM; and intelligent analytics are necessary to determine what data is most relevant (e.g., universal interface semantics for data).

Data systems need to be coupled with cost-effective sensing and control systems that support PHM. Control systems should provide real-time, closed-loop feedback and be flexible (adjustable); these can support 'smart' or intelligent machines that are self-aware and ultimately have the ability to self-adjust based on PHM inputs. Self-aware sensors and/or measurement systems will be needed to ensure data quality meets requirements for actionable decision-making.

Integration of PHM into enterprise-wide functions (planning, production, resource allocation, etc.) is another desirable capability. Enterprise planning machine data acquisition could encompass machine work allocation, machine maintenance optimization, supplies ordering, resource planning, and more. This would support user-friendly decision tools that enable just-in-time scheduling for problem avoidance. 


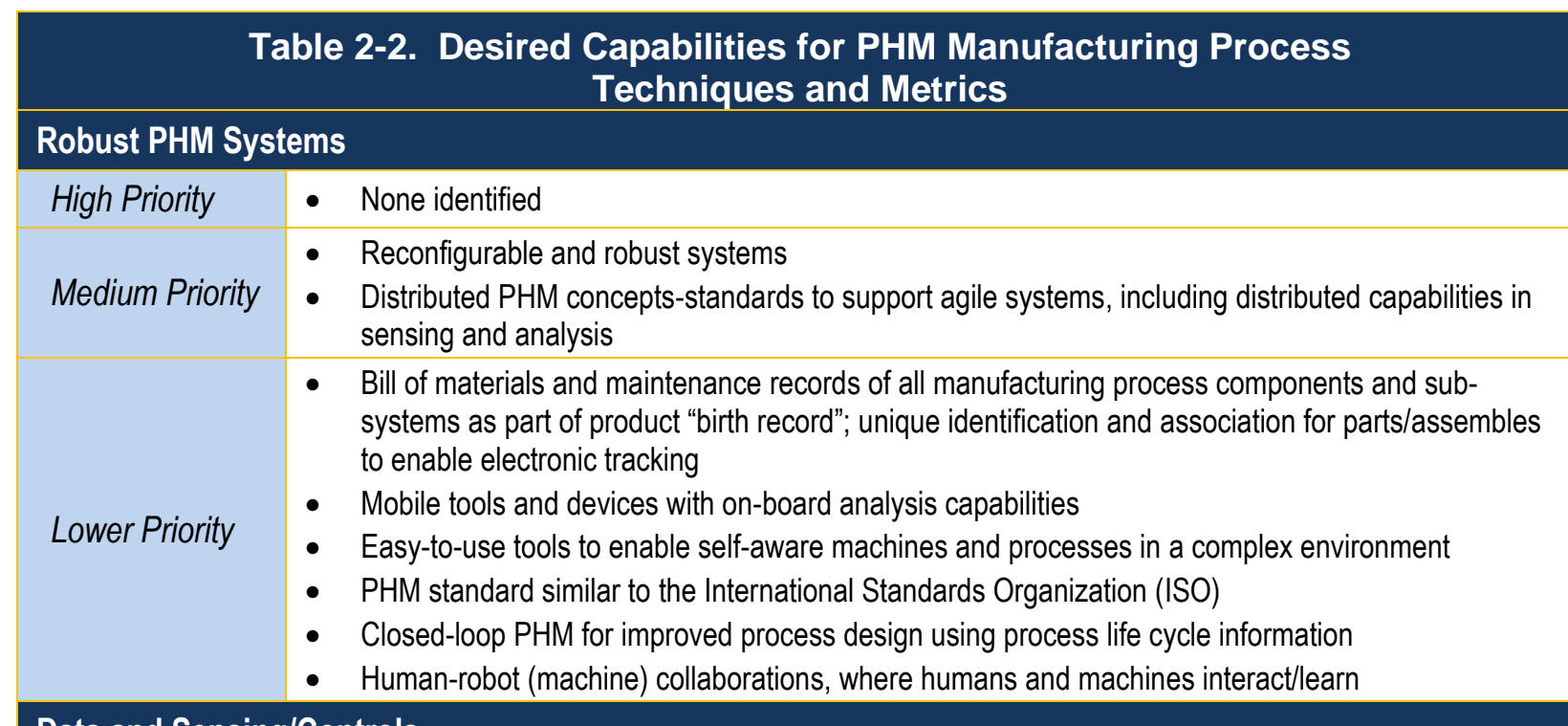

Data and Sensing/Controls

\begin{tabular}{|c|c|}
\hline High Priority & - None identified \\
\hline Medium Priority & $\begin{array}{l}\text { - Advanced data collection, management, and analytics for machine and process data, including 'Big } \\
\text { Data' (acquisition, storage, universal machine data/communications interfaces, data standards, } \\
\text { seamless data flow/analytics) } \\
\text { - Integrated, low-cost, lower-level DAQ systems coupled with cost-effective sensing, diagnostic and } \\
\text { prognostic systems and devices } \\
\text { - Integration of design, control, production, planning, product knowledge/information and diagnostics } \\
\text { into PHM (enterprise planning machine DAQ) } \\
\text { - Real-time, closed-loop feedback and adjustable control of manufacturing process supporting } \\
\text { intelligent machines that are self-aware and self-adjusting } \\
\text { - Cybersecurity protocols for sensors, network and development }\end{array}$ \\
\hline Lower Priority & $\begin{array}{l}\text { - } \quad \text { Black box data capture at lower system levels } \\
\text { - } \quad \text { Personalized processes that adapt to incoming variation in parts } \\
\text { - } \quad \text { Metrology and sensing from image/video data (e.g., camera inventory); with perception beyond } \\
\text { - } \quad \text { Pattern recognition, color intensity, etc.) }\end{array}$ \\
\hline \multicolumn{2}{|l|}{ Models } \\
\hline High Priority & - $\quad$ None identified \\
\hline Medium Priority & $\begin{array}{l}\text { - Combinable, physics-based operating models of common manufacturing components (e.g., pumps, } \\
\text { motors, pallet stop, paint spray) } \\
\text { - Combined physics-based and data-driven approaches }\end{array}$ \\
\hline Lower Priority & - None identified \\
\hline \multicolumn{2}{|l|}{ Metrics } \\
\hline High Priority & - None identified \\
\hline Medium Priority & $\begin{array}{l}\text { - } \quad \text { Accuracy of PHM (e.g., improved uptime and availability, reliability, throughput availability) } \\
\text { - } \quad \text { Statistics supporting metrics (e.g., fail-over rates, how long system can run without failure, } \\
\text { - } \quad \text { Flexility, longevity of sensor) } \\
\text { - } \quad \text { Linking of specific technical parameters and high-level KPIs } \\
\text { - } \quad \text { Metrics tied to specific goals (e.g., near zero disruption) }\end{array}$ \\
\hline
\end{tabular}




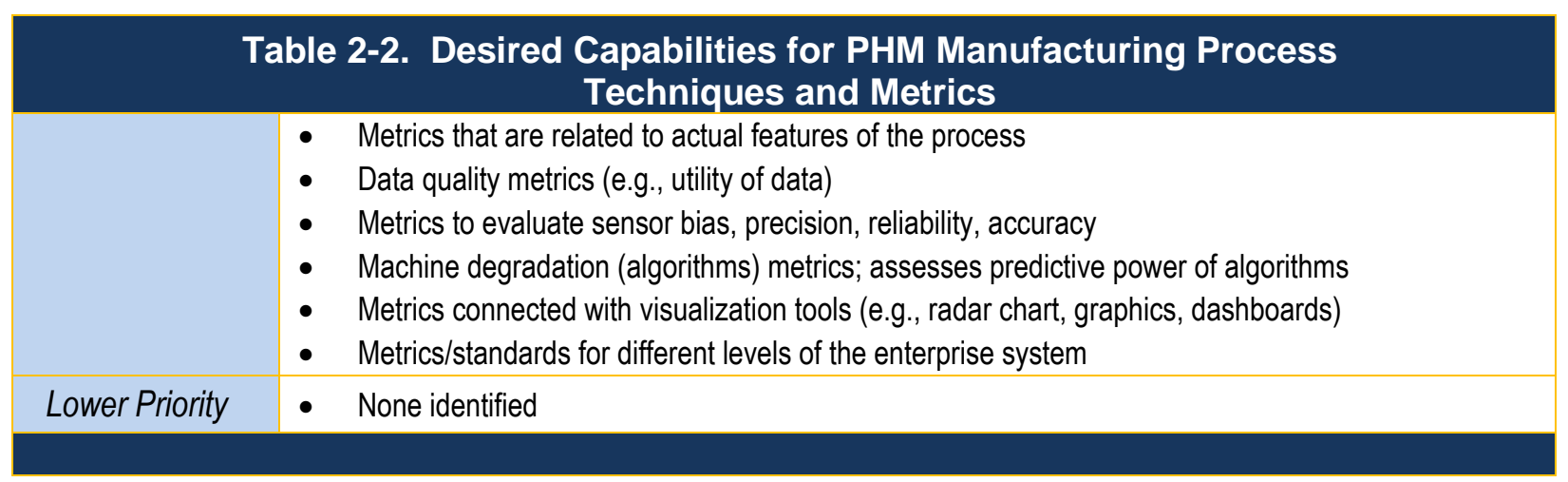

\section{Metrics}

A range of metrics were identified as supportive of PHM. Key characteristics of the metrics shown in Table 2.2 include measurement of:

- overall accuracy of PHM systems

- flexibility (i.e., ability to adjust metrics as needed)

- performance related to goals, technical parameters, or KPIs

- unique machine/process features

- data quality and utility

- $\quad$ sensor performance and utility

Metrics are recommended at many levels, from machine to enterprise. New capabilities will be needed to enable measurement of some of the parameters feeding into key metrics.

\subsection{Challenges and Barriers}

A number of challenges and barriers were identified that impede the effectiveness of PHM and development of advanced PHM for smart manufacturing. These are outlined in Table 2.3 and summarized below.

\section{Data and Sensing/Controls}

Data collection and extraction of useful information from data are impacted by a number of challenges. Interoperability between systems (e.g., sensors, data, communications, machines) remains a significant issue and needs to be addressed to enable further advances in PHM for smart manufacturing. The quality, utility, and availability of data are insufficient for numerous reasons. Automated data collection is limited, which means the captured data can be unreliable. Continued proliferation of manual processes is a contributor; data collected from manual process is generally low resolution and inaccurate, making it difficult to build a good picture of the overall process. Manual processes typically require expert human setup, tuning, configuration, and interpretation of data.

While technology exists to collect data, effective methods are not always available to track or interpret the data (or translate the data into actionable information). New technologies and methods are required to fill this gap. Sharing of PHM data and best practices is also problematic; disparate data formats, standards, 
and customization of PHM make it difficult to share information among companies and even within enterprises.

\section{Predictive Models}

Prognostic techniques are relatively immature (i.e., they are still in the development stage), so models may lack sophistication, validation, and applicability. A major challenge for predictive models to support PHM is the lack of good data for validation of prediction (e.g., interpreting what deviation from baseline means). Models for prognostics/prediction, especially those linked to highly-interconnected systems with multiple components, are inherently complex and more difficult to integrate and validate. Networked, predictive machine diagnostics are significantly lacking today.

\section{Table 2-3. Barriers / Challenges for PHM Manufacturing Process
Techniques and Metrics}

\begin{tabular}{|c|c|}
\hline High Priority & - Lack of interoperability between sensors, data formats, and communications \\
\hline Medium Priority & $\begin{array}{l}\text { - Data meaning that is lost rather than captured } \\
\text { - } \quad \text { - Pack of real-time data } \\
\text { from manualivens of manual processes in some industries; low resolution and inaccuracy of data } \\
\text { manual processes } \\
\text { - Technology exists (e.g., accelerometer) but good methods to track/interpret data are lacking } \\
\text { - Limited sharing of PHM best practices, data, success stories, and training due to disparate } \\
\text { standards and information formats and business-sensitivity; customization of manufacturing } \\
\text { systems contributes to low re-usability of PHM analytics and ability to share results } \\
\text { - Lack of flexibility of design of manufacturing systems, DAQ systems, software to enable trade- } \\
\text { offs for flexibility and robustness }\end{array}$ \\
\hline Lower Priority & $\begin{array}{l}\text { - Lack of decision support for actionable/PHM insights for maintenance technicians and non- } \\
\text { technical operators } \\
\text { - Lack of off-the-shelf PHM products, technologies, and devices that can integrate sensor and } \\
\text { other data for PHM; economy of scale is lacking }\end{array}$ \\
\hline \multicolumn{2}{|c|}{ Predictive Models } \\
\hline High Priority & - Early development stage of prognostics; cost-benefits are unclear \\
\hline Medium Priority & $\begin{array}{l}\text { - Lab experiments and validations that do not match real environment tests } \\
\text { - Limits to prognostic and predictive power - models of processes are incomplete, not well } \\
\text { validated, and lack detail at the right level for prediction } \\
\text { - } \quad \text { Complexity of systems modeling } \\
\text { - Lack of networked, predictive machine diagnostics }\end{array}$ \\
\hline Lower Priority & - None identified \\
\hline
\end{tabular}




\subsection{Priority Roadmap Topics}

Based on the major challenges and desired capabilities identified, three priority roadmaps were developed to support PHM manufacturing process techniques and metrics, as outlined below. Addressing these roadmaps will enable progress toward development and integration of PHM into smart manufacturing processes.

- Advanced Sensors for PHM in Smart Manufacturing - a multi-stage approach to sensor development, beginning with assessing available sensors and identifying gaps relevant to PHM applications; gaps would be addressed through development of new sensors and sensing capabilities and standards (Figure 2-1).

- PHM Data Format, Taxonomy and Architecture - development of data taxonomy and standards to support PHM across manufacturing sectors; the end result will be a warehouse of data and supporting standards for format, storage, organization, and interfaces (Figure 2-2).

- Enterprise-Wide PHM for Maintenance Planning - creates PHM systems that integrate data for maintenance planning with parts and other logistics; incorporates human machine interface (HMI) for decision support, with the potential for significant improvements in OEE (Figure 2-3). 


\section{Figure 2-1. AdVANCED SENSORS FOR PHM IN SMART MANUFACTURING}

Barriers: Limited understanding of the full spectrum of sensor capabilities, interfaces, and interoperability requirements for PHM; current PHM systems lack re-configurability, flexibility, scalability, and robustness to support dynamic re-tasking of machines, volume and process changes, and product variation typical of manufacturing enterprises.

Approach: This multi-step approach will develop an inventory of current sensors and identify gaps for PHM in terms of interoperability, scalability, flexibility, configuration, and other factors. Accessible libraries and taxonomies will then be developed for sensor use cases. Finally, multi-purpose sensors and related standards will be developed to achieve flexible and reconfigurable PHM systems.

\begin{tabular}{|c|c|c|c|}
\hline & Roadmap Action Plan & Milestones & Targets/ Capabilities \\
\hline $\begin{array}{c}1-2 \\
\text { years }\end{array}$ & $\begin{array}{l}\text { - Inventory sensors and DAQ interfaces used in / } \\
\text { needed for PHM systems (e.g., vibration, } \\
\text { temperature, torque, current) } \\
\text { - Describe/define re-configurability requirements for } \\
\text { typical manufacturing applications/operations } \\
\text { - Develop standards for data communications and } \\
\text { data analytics }\end{array}$ & $\begin{array}{l}\text { - Repository of sensor } \\
\text { capabilities } \\
\text { - Gap definitions - scalability } \\
\text { - Gap definitions - re- } \\
\text { configurability }\end{array}$ & $\begin{array}{l}\text { - } 75-80 \% \text { of sensors included in } \\
\text { survey } \\
\text { - } \text { Assessment of }>50 \% \text { of known } \\
\text { current systems } \\
\text { - } \quad \text { Pre-requisite for interoperability } \\
\text { and new systems }\end{array}$ \\
\hline
\end{tabular}

- Quantify gaps in re-configurability by development of scenarios and use cases for scalability and

3-5 interoperability

years

- Identify gaps in sensor and DAQ capability and interoperability

- Describe/define scalability requirements for various manufacturing applications

- Develop multi-purpose sensors/DAQ/interfaces for use in manufacturing PHM systems

- Develop standards for data communication, data analysis, prognostic algorithms

- Develop taxonomy of PHM systems and
- Library of use cases (full spectrum)

- $80 \%$ of plausible reconfigurations and rescaling covered
- At least 1-2 sensors for each data type; all interfaces supported in DAQ

- PHM handling warehouse of tools created
- Consensus achieved among stakeholders

- Flexible and reconfigurable PHM systems

\begin{tabular}{|c|c|c|c|c|c|}
\hline \multirow{2}{*}{\multicolumn{2}{|c|}{$\begin{array}{l}\text { Stakeholders \& Potential } \\
\text { Roles }\end{array}$}} & \multicolumn{2}{|c|}{ Relative Success Factors } & \multicolumn{2}{|c|}{ Benefits / Impacts } \\
\hline & & \multirow{3}{*}{$\begin{array}{l}\text { Time to Achieve } \\
\text { Results } \\
\text { Relative Cost of R\&D }\end{array}$} & \multirow[t]{2}{*}{ Medium } & Impact & Benefit \\
\hline PHM Users & $\begin{array}{l}\text { Provide input, validate } \\
\text { use cases }\end{array}$ & & & \multirow[t]{2}{*}{ High } & \multirow{2}{*}{$\begin{array}{l}\text { Improves } \\
\text { reliability/reduces failures: } \\
\text { Purpose of PHM }\end{array}$} \\
\hline $\begin{array}{l}\text { PHM Providers/ } \\
\text { Tech Developers }\end{array}$ & Prototyping, tool & & Medium & & \\
\hline \multirow{2}{*}{$\begin{array}{l}\text { Technology } \\
\text { Integrators }\end{array}$} & \multirow{2}{*}{$\begin{array}{l}\text { Validated test; sensors, } \\
\text { software (SW), integration }\end{array}$} & \multirow{3}{*}{$\begin{array}{l}\text { Complexity of R\&D } \\
\text { Risk Associated with } \\
\text { R\&D }\end{array}$} & High & Medium & $\begin{array}{l}\text { Reduces costs: Industry } \\
\text { payback over time }\end{array}$ \\
\hline & & & \multirow{2}{*}{ Medium } & \multirow{3}{*}{$\begin{array}{l}\text { Medium } \\
\text { High }\end{array}$} & Accelerates innovation: $\mathrm{n} / \mathrm{a}$ \\
\hline Academia & Conduct analysis, & & & & \multirow{2}{*}{$\begin{array}{l}\text { Enhances industry } \\
\text { competiveness: Improved } \\
\text { capability }\end{array}$} \\
\hline Acauemia & $\begin{array}{l}\text { develop SW, algorithms } \\
\text { Develop standards. }\end{array}$ & \multirow[t]{4}{*}{$\begin{array}{l}\text { Likelihood of } \\
\text { Success/Adoption }\end{array}$} & High (but slow) & & \\
\hline $\begin{array}{l}\text { Standards } \\
\text { Committees }\end{array}$ & $\begin{array}{l}\text { Develop standards, } \\
\text { pursue consensus }\end{array}$ & & & \multirow[t]{2}{*}{ High } & \multirow{2}{*}{$\begin{array}{l}\text { Speeds process re- } \\
\text { configurability: Enabling } \\
\text { technology }\end{array}$} \\
\hline Government & $\begin{array}{l}\text { Support efforts, provide } \\
\text { repository }\end{array}$ & & & & \\
\hline Other & $\begin{array}{l}\text { Facilitate input, standards } \\
\text { development }\end{array}$ & & & High & $\begin{array}{l}\text { Improves maintenance } \\
\text { scheduling: Purpose/ } \\
\text { function of PHM }\end{array}$ \\
\hline
\end{tabular}




\section{FIgURE 2-2. PHM DATA TAXONOMY AND ARCHITECTURE}

Barriers: Lack of interoperability for sensors/data formats and types of communication; preservation of data meaning/semantics. Approach: This roadmap will create the data taxonomy for PHM covering formats, storage, organization, semantics and other elements. Standards will be developed to support taxonomy as well as data interfaces and integration. The taxonomy and architecture will be applied to establish a data warehouse of PHM data sources.

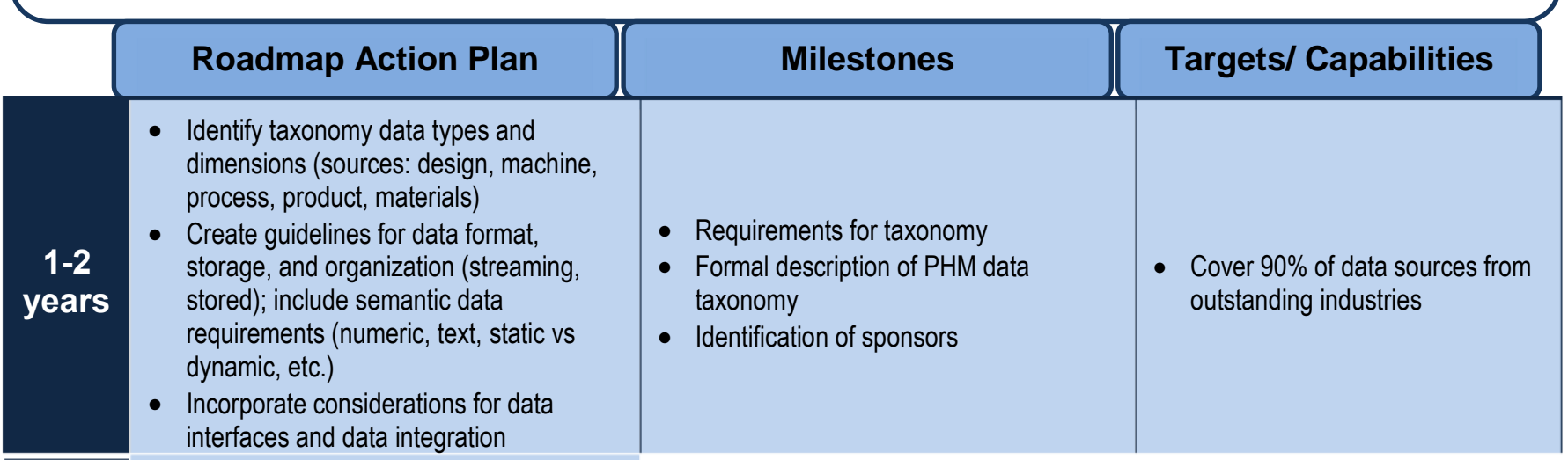

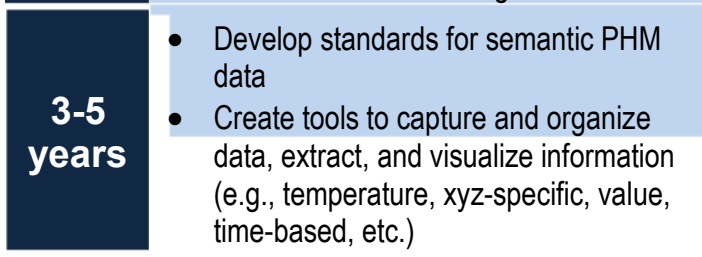

- Create, organize, and manage data repositories
- Major workgroups formed

- Taxonomy validated and tested according to use cases/pilots by participating industries

- Specification of data models

- Data warehouse creation

- Expansion of repository and data sources
- Agreement across domains/ industries

- Applicability to $75 \%$ of industry

- Used by businesses/ other organizations

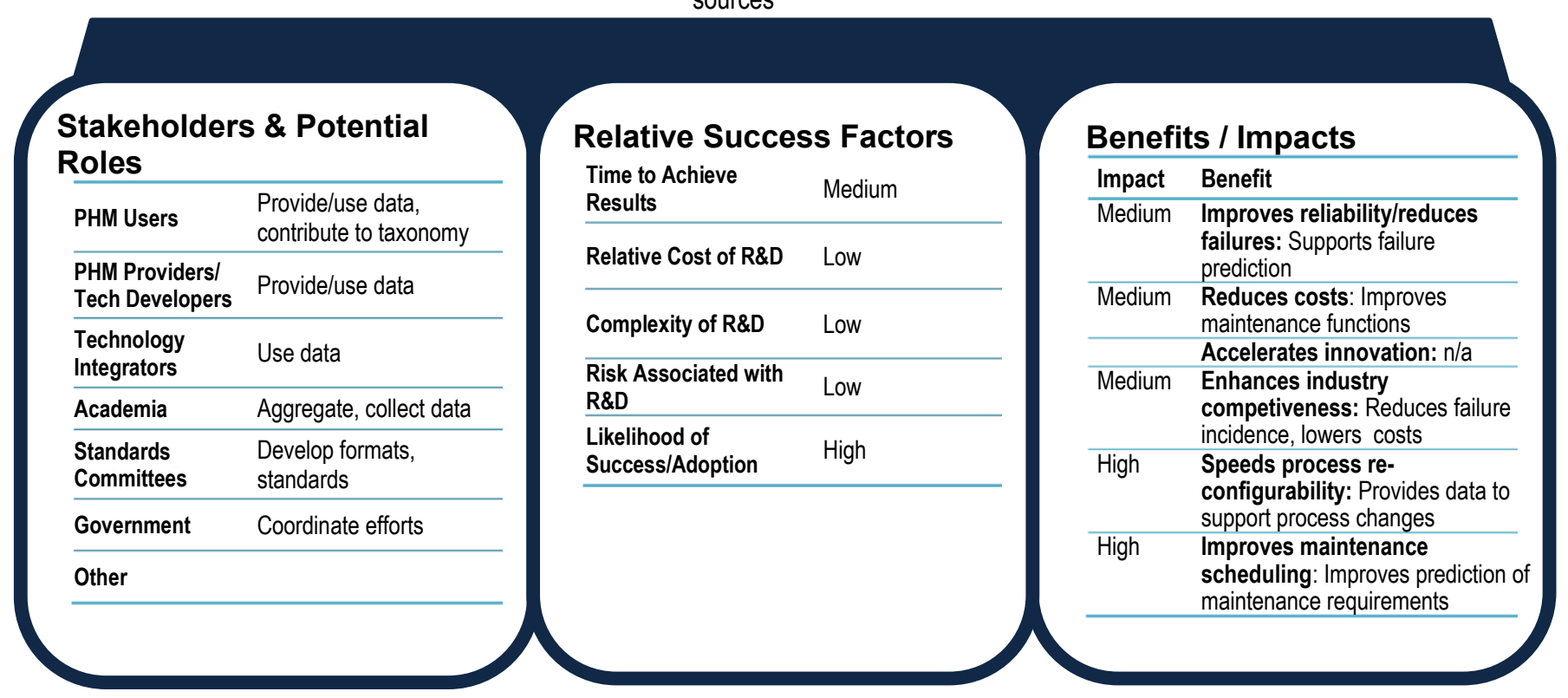




\section{Figure 2-3. Enterprise-Wide PHM for Maintenance Planning}

Barriers: Collection and analysis of data from various manufacturing sources (programmable logic controller (PLC), sensors, human). Limited methods for identifying the key data; difficulty interpreting meaning when processes change (actionable information) within context of the process change (contextualized data); lack of database with consistent format and data architecture (build, plan, definition, integration of data).

Approach: In the first phase, the impact of system elements with respect to breakdown will be prioritized and a maintenance planning PHM methodology developed. The second phase would integrate enterprise-wide logistics and real-time information. Algorithms will be needed to support multi-objective optimization with changing inputs and elements, analysis for performance assessment, and finally feedback and control.

\begin{tabular}{|c|c|c|c|}
\hline & Roadmap Action Plan & Milestones & Targets/ Capabilities \\
\hline $\begin{array}{c}1-2 \\
\text { years }\end{array}$ & $\begin{array}{l}\text { Investigate current state-of-the-art } \\
\text { for functional descriptions of } \\
\text { system, manufacturing data flow }\end{array}$ & $\begin{array}{l}\text { - Functional description of system elements with } \\
\text { inputs/outputs, connections to all elements and } \\
\text { contextual information } \\
\text { - Testbed demonstration of data architecture with } \\
\text { data collection (system and sub-system) } \\
\text { - Initial outlining of analysis and optimization linking } \\
\text { logistics and factory data }\end{array}$ & $\begin{array}{l}\text { - At least } 1 \text { year of data } \\
\text { collected for at least } 1 \\
\text { manufacturing system (or } \\
\text { large sub-systems) }\end{array}$ \\
\hline $\begin{array}{c}3-5 \\
\text { years }\end{array}$ & $\begin{array}{l}\text { - Develop algorithms using test-bed } \\
\text { data }\end{array}$ & $\begin{array}{l}\text { - Algorithms for maintenance planning } \\
\text { - HMI visualization/dashboard for decision-support, } \\
\text { presenting, and optimization of solutions } \\
\text { - Demonstration of human machine interface (HMI) } \\
\text { tools user algorithms }\end{array}$ & - $5 \%$ improvement in OEE \\
\hline $\begin{array}{c}5+ \\
\text { years }\end{array}$ & $\begin{array}{l}\text { Develop GPS of where plant } \\
\text { elements, including tools, are } \\
\text { located in the plant; develop RFID } \\
\text { of parts for tracking across-the- } \\
\text { board consistently }\end{array}$ & $\begin{array}{l}\text { - Algorithms for near real-time actionable } \\
\text { information (in-process) and maintenance } \\
\text { planning (post and pre-process); industry } 5.0 \\
\text { - } \quad \text { Artificial-intelligence for self-monitoring } \\
\text { - } \quad \text { HMI tools for near real-time Q\&A using functional, } \\
\text { contextualized data }\end{array}$ & - $10 \%$ improvement in OEE \\
\hline
\end{tabular}

Stakeholders \& Potential Roles

\begin{tabular}{ll}
\hline PHM Users & $\begin{array}{l}\text { Provide data, test/deploy } \\
\text { system, develop metrics }\end{array}$ \\
\hline $\begin{array}{l}\text { PHM Providers/ } \\
\text { Tech Developers }\end{array}$ & $\begin{array}{l}\text { Provide data, test /deploy } \\
\text { system, develop metrics }\end{array}$ \\
\hline $\begin{array}{l}\text { Technology } \\
\text { Integrators }\end{array}$ & Test/deploy system \\
\hline Academia & Develop/test algorithms \\
\hline $\begin{array}{l}\text { Standards } \\
\text { Committees }\end{array}$ & n/a \\
\hline Government & Develop/test algorithms \\
\hline
\end{tabular}

Relative Success Factors

Time to Achieve

Results

Relative Cost of R\&D Medium

Complexity of R\&D Medium

Risk Associated with

R\&D Low

Likelihood of

Success/Adoption
High

\section{Benefits / Impacts}

\section{Impact Benefit}

Medium Improves reliability/reduces failures: Supports better failure prediction tools

Low Reduces costs: Balances costs enterprise-wide

Low Accelerates innovation: Eases adoption of new technology Enhances industry competiveness: Reduces maintenance costs, bottom line Speeds process reconfigurability: Support agility (and process changes)

High Improves maintenance scheduling: Supports better maintenance planning 


\section{PHM Performance Assessment}

\subsection{Overview}

Section 3 presents the Roadmapping Workshop results of a breakout group focused on the topic of PHM Performance Assessment. This includes assessing the performance of the PHM system itself, as well as the supporting technologies, data, and metrics needed to support such assessments. The section summarizes the breakout group's discussions and activities. Participants identified goals, desired capabilities, challenges and barriers, and priority roadmap topics. A list of participants from all breakout groups is shown in Appendix A.

By predicting reliability, and monitoring and recording health relative to normal operating conditions, PHM can help manage the safety and cost of manufacturing systems while improving manufacturing operations and product quality. A key aspect of PHM is assessing how well prognostics are performing their function.

Effectively assessing the performance of PHM requires standards, measurement capabilities, and systemrelevant metrics. The objective is to assess the performance of PHM systems relative to their predictive and monitoring capabilities, identification of failure or other events, and success in supporting effective maintenance practices and/or the application of corrective actions. The performance, in many cases, is dependent on the accuracy of the algorithms and predictive models underlying the PHM system.

One example is the accuracy of the prognostic horizon, which is defined as the difference between the time index when prognostic predictions first meet the specified performance criteria and the time for End of Life (EoL). Measuring effectiveness of the prognostic horizon could require examining whether the algorithm predicts within desired accuracy around the EoL. Another example is measuring whether algorithms stay within desired performance levels relative to specified parameters.

Measuring PHM performance within a smart

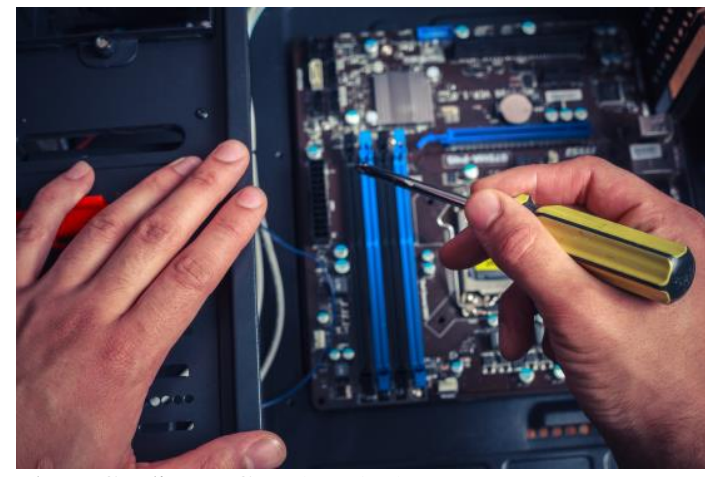

Photo Credit: DPC \#65797175 manufacturing environment creates new challenges and complexities, as systems become highly interconnected and interdependent. New metrics and methods for measuring PHM performance will be needed as smart manufacturing systems evolve, including ways to measure PHM at the equipment level, as well as at the overall system level. 


\subsection{Goals}

Near-term goals for PHM performance assessment focus on evaluation of PHM as well as incorporating the key performance characteristics that are critical to manufacturing, including failure prediction/ recovery, defect reduction, and early detection of events. A supporting goal is developing models/simulations that can effectively identify where critical points are in the manufacturing process for PHM implementation. In the mid-term, it is desirable to ensure that PHM systems can achieve higher fault predictions of components or systems than currently possible today. Long-term goals for assessing PHM performance include evaluating specific characteristics, such as the ability for autonomous recovery from failure/fault conditions. Comprehensive understanding of the data needed to assess PHM performance is another long-term goal.

\begin{tabular}{|c|c|c|}
\hline $\begin{array}{l}\text { NEAR-TERM } \\
\text { (1 to } 2 \text { Years) }\end{array}$ & $\begin{array}{c}\text { MID-TERM } \\
\text { (3 to } 5 \text { Years) }\end{array}$ & $\begin{array}{c}\text { LONG-TERM } \\
\text { (5 + Years) }\end{array}$ \\
\hline $\begin{array}{l}\text { Desired PHM Performance } \\
\text { Characteristics/Metrics } \\
\text { - Identification of data or performance } \\
\text { characteristics critical to smart } \\
\text { manufacturing and incorporation into } \\
\text { PHM performance assessment } \\
\text { - Ability to predict failure of the } \\
\text { component under study within } 10 \% \\
\text { of its entire life } \\
\text { PHM-Related Equipment } \\
\text { Effectiveness/ Monitoring } \\
\text { Technology } \\
\text { - Reduction in number of defective } \\
\text { parts at the end of line by accurate } \\
\text { anomaly detection and diagnosis } \\
\text { (yield improvement of } 20 \% \text { ) } \\
\text { - Model-based identification of critical } \\
\text { areas in a manufacturing system } \\
\text { where PHM capability is needed; } \\
\text { and integration in PHM performance } \\
\text { assessment }\end{array}$ & $\begin{array}{l}\text { Desired PHM Performance } \\
\text { Characteristics/Metrics } \\
\text { - Ability for fault prediction at } \\
\text { component and system level, } \\
\text { with greater than } 50 \% \text { likelihood } \\
\\
\text { PHM-Related Equipment } \\
\text { Effectiveness/ Monitoring } \\
\text { Technology } \\
\text { - MTConnect available in } 100 \% \text { of } \\
\text { new machine tools to aid in PHM } \\
\text { performance assessment and } \\
\text { overall PHM system use } \\
\text { - Ability to produce actionable } \\
\text { data to optimize repair/replace } \\
\text { schedules }\end{array}$ & $\begin{array}{l}\text { Desired PHM Performance } \\
\text { Characteristics/Metrics } \\
\text { - Ability for autonomous recovery from fault } \\
\text { conditions } \\
\text { - Good understanding of how much/type of } \\
\text { data needed to test the PHM system } \\
\text { PHM-Related Equipment Effectiveness/ } \\
\text { Monitoring Technology } \\
\text { - Effectiveness of self-prognostic machines } \\
\text { for all critical manufacturers } \\
\text { - Integration of real-time analysis control } \\
\text { for assessment of PHM } \\
\text { Design and Life Cycle PHM for } \\
\text { Performance Assurance } \\
\text { - PHM system designed into the product to } \\
\text { enhance performance } \\
\text { - Integration of PHM into the systems } \\
\text { engineering process for better } \\
\text { performance assurance } \\
\text { - Effective PHM svstem validation }\end{array}$ \\
\hline
\end{tabular}

\subsection{Desired Capabilities}

The desired capabilities identified for PHM performance assessment are outlined in Table 3.2 and summarized below. These encompass performance assessment of PHM systems at the equipment and system levels; response to failure modes; and validation of the accuracy of PHM techniques.

\section{Performance of PHM, Diagnostics, and Maintenance}

The most desired capability for measuring the performance of health monitoring, diagnostic, prognostic, and maintenance systems and components is the availability of pre-failure prognostic metrics. Other 
important capabilities include making non-smart-manufacturing equipment intelligent enough to allow for monitoring and controlling capabilities, and cost-effectively modeling performance assessment before developing designs.

\section{Performance of PHM in Response to Sources of Failure}

When measuring the performance of PHM systems in response to sources of failure, one of the most desirable capabilities is the integration of manufacturing, maintenance and usage data, starting from the component level. It is important to not only consider the impacts of PHM at the equipment level, but also the performance of the overall system. Requisite data resources are needed to support these capabilities, such as an information repository of failure criteria, which could be used to inform and compare PHM systems.

\section{Verification and Validation of Manufacturing PHM Techniques}

Effective verification and validation of manufacturing PHM techniques could also benefit from more extensive and comprehensive data, as well as tools, models, methodologies, standard platforms for information dissemination, and guidelines for verification and validation.

\begin{tabular}{|c|c|}
\hline \multicolumn{2}{|c|}{ Performance of PHM, Diagnostics, and Maintenance } \\
\hline High Priority & - $\quad$ Pre-failure prognostic performance metric \\
\hline Medium Priority & $\begin{array}{l}\text { - } \quad \text { Ability to easily make non-smart-manufacturing equipment smart enough to be monitored } \\
\text { - } \quad \text { Model for assessment of performance prior to design } \\
\text { - } \quad \text { Industry standard digital sensor interface that are control system agnostic } \\
\text { - } \quad \text { Manufacturing equivalent of electronic health records } \\
\text { - } \quad \text { Usage-based and physics-based performance metrics (model-based systems) views }\end{array}$ \\
\hline Lower Priority & $\begin{array}{l}\text { - Standard data models for process and equipment use by various data collection systems. } \\
\text { - Seamless synchronization for process changes. } \\
\text { - } \quad \text { Probabilistic approach to characterize the effect of PHM (safety, availability, production, etc.). } \\
\text { - }\end{array}$ \\
\hline
\end{tabular}

\section{Performance of the PHM System in Response to Sources of Failure}
High Priority
- Integration of manufacturing, maintenance and usage data, starting from the component level
- Consideration of the impacts of PHM in equipment level as well as overall system performance
- Self-learning capabilities
- Database of failure criteria to be used to inform and compare the PHM system
Medium Priority
- Better hardware for simulating PHM performance and uncertainty to drastically reduce computational cost
- Standards and guidelines to ensure the success of PHM in the future of the manufacturing industry and our everyday life
- Modeling the impact on system performance of PHM results - Identify possible response strategy and time requirement

\section{Lower Priority}
- Systematic, standardized, and effective method for conducting or modeling accelerated life testing
- Plant-wide key performance indicator; PHM tools for production and asset utilization targets 


\begin{tabular}{|c|c|}
\hline \multicolumn{2}{|r|}{ Table 3-2. Desired Capabilities for PHM Performance Assessment } \\
\hline High Priority & - $\quad$ None identified \\
\hline Medium Priority & $\begin{array}{l}\text { - Verification and validation performed with limited data } \\
\text { - Supply-chain-wide standards, techniques, and tools for PHM overall effectiveness index } \\
\text { - Guidelines and standards for verification and validation of manufacturing PHM techniques } \\
\text { - } \quad \text { Better methods to identify systems of interest (not rate occurrence of failure or effect of } \\
\text { - } \text { downtime) } \\
\text { - Standard platform for central domain experts to develop and teach diagnosis/prognostic } \\
\text { - Methorithms across enterprise } \\
\text { - Systems can connect with each other, i.e., Supervisory Control and Data Acquisition/ } \\
\text { Computerized Maintenance Management System (SCADA/CMMS), CMMS with quality - } \\
\text { relational data }\end{array}$ \\
\hline Lower Priority & - Methodology to measure PHM system performance for new systems \\
\hline
\end{tabular}

\subsection{Challenges and Barriers}

Achieving the desirable capabilities and needs for PHM performance assessment requires overcoming several challenges and barriers, outlined in Table 3-3 and summarized below.

\section{Availability of Data}

The availability (or lack thereof) of historical data necessary to create diagnostic and prognostic models presents a challenge. It is difficult to accurately assess performance without "ground truth" for online performance metrics. Issues contributing to the lack of data include the costs associated with obtaining failure data, such as damaging systems and equipment, and a reluctance towards data sharing (partly because of organizations wanting to maintain a competitive advantage).

\section{Standards}

Another issue contributing to the lack of available data and hindering the idea of creating industry-wide datasets for developing predictive models is a lack of consistency in the classification of equipment and failure codes. This is not only a problem for classifying information across multiple industrial facilities and organizations, but it also exists within companies themselves.

\section{Design Challenges}

It is difficult to gauge the tradeoffs between making design changes versus making partial system upgrades. The maintenance of PHM solutions should be taken into consideration when making decisions between the two choices.

\section{Support and Awareness}

The widespread use of PHM techniques could increase with greater support from leadership and the manufacturing community. The lack of buy-in is accompanied by low industry-PHM awareness, experience, and training needed to apply principles and tools. 


\section{Table 3-3. Barriers and Challenges for PHM Performance Assessment}

\section{Availability of Data}

\begin{tabular}{|c|c|}
\hline High Priority & $\begin{array}{l}\text { - Sparse datasets to develop models for diagnostics and prognostics, specifically for failing } \\
\text { equipment, and good historical diagnostic information } \\
\text { - } \quad \text { Trying to assess performance without ground truth for online performance metrics }\end{array}$ \\
\hline Medium Priority & $\begin{array}{l}\text { - Lack of cost effective means to get test data (run-to-failure) } \\
\text { - } \quad \text { Fear of sharing data; "give to get" maintenance data model could work } \\
\text { - } \quad \text { Lack of comprehensive, integrated data from components, and the assembled asset } \\
\text { minimizing possibible interruptions) }\end{array}$ \\
\hline Lower Priority & - Lack of agreement on validated sensors and software and an agreed upon standard \\
\hline \multicolumn{2}{|l|}{ Standards } \\
\hline High Priority & - No consistency in classification of equipment, failure codes, etc., even within a company \\
\hline Medium Priority & $\begin{array}{l}\text { - Inventory to sales ratio is an hidden/undisclosed control group of manufacturing } \\
\text { - } \quad \text { Lack of failure/fault characterization through modeling, simulation, and accelerated testing } \\
\text { - } \quad \text { Lack of independent evidence to support standards development } \\
\text { - Lack of technology and standards for small and medium enterprises - common (broad market) }\end{array}$ \\
\hline Lower Priority & - $\quad$ None identified \\
\hline \multicolumn{2}{|c|}{ Design Challenges } \\
\hline High Priority & - $\quad$ None identified \\
\hline Medium Priority & $\begin{array}{l}\text { - } \quad \text { Design changes versus partial system upgrades (maintenance of PHM solution) } \\
\text { - } \quad \text { Lack of methods to roll-up component-specific performance to the system level } \\
\text { - Lack of more consolidated solutions for simplicity }\end{array}$ \\
\hline Lower Priority & - Use of "proven" PHM platforms integrated with other manufacturing operations/design \\
\hline \multicolumn{2}{|c|}{ Support and Awareness } \\
\hline High Priority & - Lack of continuous leadership/community support for implementation of PHM techniques \\
\hline Medium Priority & $\begin{array}{l}\text { - } \quad \text { Low level of industry PHM awareness (education, skill, experience) to apply principles and tools } \\
\text { - } \quad \text { Lack of communication and understanding of what's available and capabilities } \\
\text { - } \quad \text { Lack of buy-in and support from small and medium companies (risky and expensive) }\end{array}$ \\
\hline Lower Priority & $\begin{array}{l}\text { - } \quad \text { "Closing the loop" on recommendations; difficult to discover root cause/field action } \\
\text { - Tradition and culture that limit innovations (e.g., doing things the same way for generations) }\end{array}$ \\
\hline
\end{tabular}




\subsection{Priority Roadmap Topics}

Based on the major challenges and desired capabilities identified, six priority roadmaps were developed, as outlined below.

- Overarching Architecture Framework for PHM with Standards and KPIs - Perform industryspecific benchmarks to define the current state, identify key performance indicators, and define the standard PHM architecture. The framework could serve as a valuable reference for the smart manufacturing industry.

- Cost Model for PHM Performance - Develop a cost-benefit analysis to better understand when and where to apply PHM. The resulting model would take into consideration the costs associated with equipment failures, collateral damage, lost revenue from machines being out of service, and the cost of implementing PHM systems.

- Identification of PHM Performance Metrics - Develop online, prognostic performance metrics to evaluate algorithm performance during manufacturing operation before system or component failure, and inform control operations. These metrics would be identified at the component and system levels and could help reduce equipment failure and improve asset utilization.

- Taxonomy for Applications - Taxonomy to provide consistency in the definition of components, standard testing procedures, and interpretations of failure events. The resulting glossary could improve internal and external communications and help obtain buy-in from management and the manufacturing community.

- Determination of PHM Data and Information Needs - Collection of information about the most prevalent characteristics for a PHM system, existing data collection strategies, and major causes for failure and downtime. The effort would seek to provide standard inputs for PHM configuration, architecture, and development and the resulting information repository could help increase the adoption of PHM within the manufacturing community.

- Failure Data for Prognostics and Diagnostics - Methods for developing and collecting data to support failure prognostics and diagnostics. Methodologies could result in a common database to validate and verify diagnostics and prognostics of components or systems and facilitate standards development. 


\section{Figure 3-1. OVERARching ARchitecture FramewORK For PHM WITH STANDARDS AND KPIS}

Barriers: Current status of the PHM framework in various industries is unclear; a PHM framework that includes standards is lacking, leading to inconsistencies in performance assessment.

Approach: The development of the framework would involve: 1) performing a benchmark for current state, 2) cataloging Key Performance Indicators (KPIs), and 3) defining PHM standard architecture and traceability. A standard framework will provide consistency in assessment of PHM performance and support new standards development.

\begin{tabular}{|c|c|c|c|}
\hline & Roadmap Action Plan & Milestones & Targets/ Capabilities \\
\hline $\begin{array}{c}1-2 \\
\text { years }\end{array}$ & $\begin{array}{l}\text { Benchmark the current state of } \\
\text { machine monitoring in select } \\
\text { industries, such as oil and gas, } \\
\text { automotive, aerospace, and } \\
\text { pharma/medical }\end{array}$ & $\begin{array}{l}\text { Identification of two key PHM scenarios to } \\
\text { benchmark from each industry, non-advanced and } \\
\text { advanced }\end{array}$ & $\begin{array}{l}\text { - } 80 \% \text { of the benchmarks } \\
\text { completed }\end{array}$ \\
\hline $\begin{array}{l}3-5 \\
\text { years }\end{array}$ & $\begin{array}{l}\text { Catalog the KPls in the above } \\
\text { industries and map-out common } \\
\text { trends for prognostics and } \\
\text { diagnostics }\end{array}$ & $\begin{array}{l}\text { Catalog of KPIs based on benchmarking and best } \\
\text { practices }\end{array}$ & $\begin{array}{l}\text { - Published catalog that is } \\
\text { accepted by } 30 \% \text { of industry }\end{array}$ \\
\hline $\begin{array}{c}5+ \\
\text { years }\end{array}$ & $\begin{array}{l}\text { Define the standard PHM } \\
\text { architecture } \\
\text { Create methods for asset traceability } \\
\text { and history recording }\end{array}$ & $\begin{array}{l}\text { - Published system architecture and data flow } \\
\text { - Playbook for machine monitoring/benchmarking } \\
\text { - ISO standard traceability }\end{array}$ & - Creation of ISO documents \\
\hline
\end{tabular}

Stakeholders \& Potential Roles

\begin{tabular}{ll}
\hline PHM Users & $\begin{array}{l}\text { Champion needs, } \\
\text { problems, current state }\end{array}$ \\
\hline $\begin{array}{l}\text { PHM Providers/ } \\
\text { Tech Developers }\end{array}$ & $\begin{array}{l}\text { Provide historical } \\
\text { successes and current } \\
\text { capabilities }\end{array}$ \\
\hline $\begin{array}{l}\text { Technology } \\
\text { Integrators }\end{array}$ & $\begin{array}{l}\text { Adopt standard input on } \\
\text { architecture }\end{array}$ \\
\hline Academia & $\begin{array}{l}\text { Conduct literature studies, } \\
\text { perform due diligence }\end{array}$ \\
\hline $\begin{array}{l}\text { Standards } \\
\text { Committees }\end{array}$ & Accept and publish \\
\hline Government & Provide technical support \\
\hline
\end{tabular}

Relative Success Factors

Time to Achieve Medium
Results

Relative Cost of R\&D Low

Complexity of R\&D LoW

Risk Associated with Medium

$\begin{array}{ll}\text { R\&D } & \text { Medium } \\ \text { Likelihood of } & \end{array}$

Success/Adoption

Medium
Benefits / Impacts

\begin{tabular}{ll}
\hline Impact & Benefit \\
\hline High & $\begin{array}{l}\text { Improves reliability/reduces } \\
\text { failures }\end{array}$ \\
\hline High & Reduces costs \\
\hline Medium & Accelerates innovation \\
\hline High & $\begin{array}{l}\text { Enhances industry } \\
\text { competiveness }\end{array}$ \\
\hline Low & $\begin{array}{l}\text { Speeds process re- } \\
\text { configurability }\end{array}$ \\
& $\begin{array}{l}\text { Improves maintenance } \\
\text { scheduling }\end{array}$ \\
\hline
\end{tabular}




\section{Figure 3-2. COST MOdel FOR PHM PeRformance}

Barriers: Subjectivity inherent in gathering risk data and predicting the value of a PHM system, especially since the complexity and differences of machines varies widely.

Approach: Cost data is needed for assessment of equipment failures, collateral damage, and lost opportunity (lost revenue from machines being out of service). In addition, the cost of implementing a PHM system must be evaluated. A database of cost information will be developed to support ROI determinations and comparison by fault modes.

\begin{tabular}{|c|c|c|c|}
\hline & Roadmap Action Plan & Milestones & Targets/ Capabilities \\
\hline $\begin{array}{c}1-2 \\
\text { years }\end{array}$ & $\begin{array}{l}\text { Gather data on costs resulting from: } \\
\text { machine failures, machine repair, } \\
\text { damage to products/surroundings, } \\
\text { and factory downtime } \\
\text { Determine cost of PHM systems } \\
\text { development and integration }\end{array}$ & $\begin{array}{l}\text { Comparison of failure cost to PHM cost to determine } \\
\text { return on investment (ROI) } \\
\text { - Determination of reasonable amortization } \\
\text { Database of cost information and failure likelihood } \\
\text { broken down by component }\end{array}$ & \multirow{3}{*}{$\begin{array}{l}\text { Cost models for two to three } \\
\text { common machines } \\
\text { Collection and analysis of } \\
\text { sufficient data to determine a } \\
\text { pattern for ROI calculations and } \\
\text { build the business case for PHM }\end{array}$} \\
\hline $\begin{array}{c}3-5 \\
\text { years }\end{array}$ & $\begin{array}{l}\text { Refine model, broken down by fault } \\
\text { mode } \\
\text { - Expand list of machinery }\end{array}$ & $\begin{array}{l}\text { Same as above, but more detailed } \\
\text { - List of fault modes } \\
\text { - Assessment of performance } \\
\text { - Actions taken }\end{array}$ & \\
\hline $\begin{array}{c}5+ \\
\text { years }\end{array}$ & - Develop inverse model & - Capability for deriving requirements from ROI goals & \\
\hline
\end{tabular}

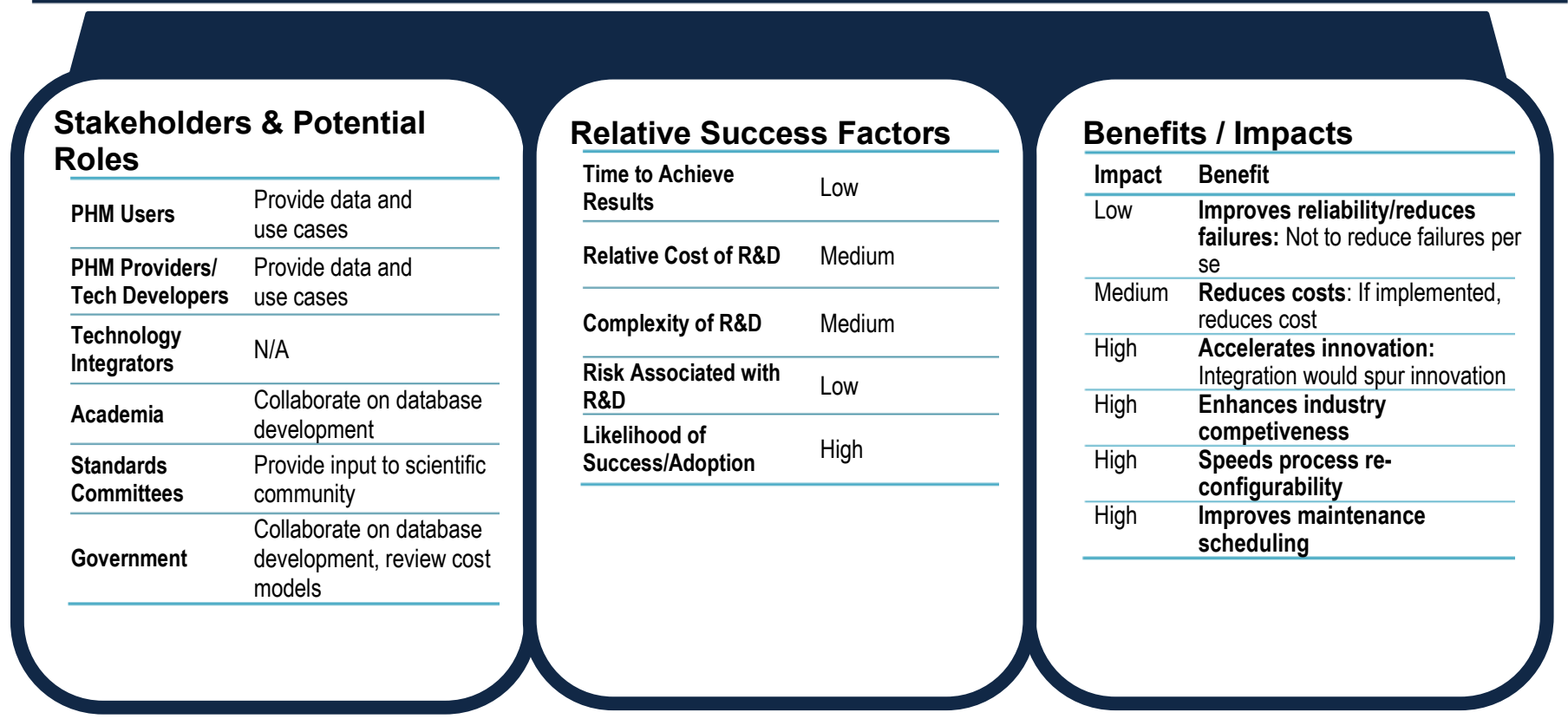




\section{FiguRE 3-3. IDENTIFICATION OF PHM PeRformance Metrics}

Barriers: Lack of performance metrics to characterize efficacy of prognostics applied to a specific system before that system fails; insufficient information on key performance metrics for manufacturing at component and system levels. Approach: Performance metrics are needed that can evaluate algorithm performance during operation (pre-failure) to inform system control and operations and maintenance planning. New performance metrics will be developed as needed. New and existing metrics will be evaluated for application to manufacturing systems.

\begin{tabular}{|c|c|c|c|}
\hline & Roadmap Action Plan & Milestones & Targets/ Capabilities \\
\hline $\begin{array}{c}1-2 \\
\text { years }\end{array}$ & $\begin{array}{l}\text { Survey existing performance metrics that } \\
\text { characterize performance of prognostic/PHM } \\
\text { system itself and effectiveness when applied to } \\
\text { system } \\
\text { - Identify necessary metrics to apply prognostics } \\
\text { to manufacturing equipment and integrate with } \\
\text { controls/O\&M planning } \\
\text { - Understand gaps between existing metrics and } \\
\text { desired metrics }\end{array}$ & $\begin{array}{l}\text { State-of-the-art and gaps assessment for } \\
\text { manufacturing PHM performance metrics }\end{array}$ & $\begin{array}{l}\text { White paper to present state-of-the-art } \\
\text { and gaps assessment }\end{array}$ \\
\hline $\begin{array}{c}3-5 \\
\text { years }\end{array}$ & $\begin{array}{l}\text { Develop missing metrics } \\
\text { (equipment, systems) and PHM algorithms } \\
\text { Investigate how to integrate performance } \\
\text { metrics in control or operations, and } \\
\text { maintenance planning systems }\end{array}$ & $\begin{array}{l}\text { New PHM performance metrics for } \\
\text { manufacturing } \\
\text { Demonstration of metrics on testbed or } \\
\text { simulation }\end{array}$ & $\begin{array}{l}\text { - Reduction of in-service failure } \\
\text { - Improved asset utilization }\end{array}$ \\
\hline $\begin{array}{c}5+ \\
\text { years }\end{array}$ & $\begin{array}{l}\text { Integrate with generic PHM architecture to } \\
\text { implement metrics } \\
\text { - Conduct demonstrations at pilot plant(s) }\end{array}$ & $\begin{array}{l}\text { Demonstration of metrics in real-world } \\
\text { application }\end{array}$ & $\begin{array}{l}\text { Integration of metrics in } \mathrm{PHM} \\
\text { framework and manufacturing plant }\end{array}$ \\
\hline
\end{tabular}

\begin{tabular}{|c|c|c|c|c|c|}
\hline \multirow{2}{*}{\multicolumn{2}{|c|}{$\begin{array}{l}\text { Stakeholders \& Potential } \\
\text { Roles }\end{array}$}} & \multicolumn{2}{|c|}{ Relative Success Factors } & \multicolumn{2}{|c|}{ Benefits / Impacts } \\
\hline & & \multirow{2}{*}{$\begin{array}{l}\text { Time to Achieve } \\
\text { Results }\end{array}$} & \multirow{2}{*}{ Medium+ } & Impact & Benefit \\
\hline \multirow{2}{*}{ PHM Users } & \multirow{2}{*}{$\begin{array}{l}\text { Provide input on needs; } \\
\text { Conduct pilot } \\
\text { demonstrations }\end{array}$} & & & \multirow[t]{3}{*}{ High } & \multirow{3}{*}{$\begin{array}{l}\text { Improves reliability/reduces } \\
\text { failures: Better decisions can be } \\
\text { made by considering PHM results } \\
\text { and performance metrics }\end{array}$} \\
\hline & & \multirow{2}{*}{ Relative Cost of R\&D } & \multirow{2}{*}{ Low/Medium } & & \\
\hline \multirow{2}{*}{$\begin{array}{l}\text { PHM Providers/ } \\
\text { Tech Developers }\end{array}$} & \multirow{2}{*}{$\begin{array}{l}\text { Develop metrics and } \\
\text { algorithms; Implement } \\
\text { metrics in PHM framework }\end{array}$} & & & & \\
\hline & & Complexity of R\&D & Medium/High & Medium & $\begin{array}{l}\text { Reduces costs: Consequence of } \\
\text { failure reduction }\end{array}$ \\
\hline $\begin{array}{l}\text { Technology } \\
\text { Integrators }\end{array}$ & $\begin{array}{l}\text { Validate and test integration } \\
\text { of metrics with framework }\end{array}$ & $\begin{array}{l}\text { Risk Associated with } \\
\text { R\&D }\end{array}$ & Medium & \multirow[t]{2}{*}{ Medium } & \multirow{2}{*}{$\begin{array}{l}\text { Accelerates innovation: Easier to } \\
\text { compare competing PHM systems } \\
\text { for a specific application } \\
\text { Integration with control supports } \\
\text { advanced control systems }\end{array}$} \\
\hline Academia & $\begin{array}{l}\text { Develop metrics and } \\
\text { algorithms; conduct testbed/ } \\
\text { simulation demonstrations }\end{array}$ & \multirow[t]{5}{*}{$\begin{array}{l}\text { Likelihood of } \\
\text { Success/Adoption }\end{array}$} & High & & \\
\hline $\begin{array}{l}\text { Standards } \\
\text { Committees }\end{array}$ & $\begin{array}{l}\text { Develop and disseminate } \\
\text { standards or guidelines for } \\
\text { metrics }\end{array}$ & & & High & $\begin{array}{l}\text { Enhances industry } \\
\text { competiveness: Improved quality } \\
\text { and productivity }\end{array}$ \\
\hline \multirow[t]{3}{*}{ Government } & Support standards & & & & $\begin{array}{l}\text { Speeds process re-configurability: } \\
\text { Some support through advanced }\end{array}$ \\
\hline & & & & & control algorithms \\
\hline & & & & High & $\begin{array}{l}\text { Improves maintenance scheduling } \\
\text { Provides more actionable information }\end{array}$ \\
\hline
\end{tabular}




\section{FIGURE 3-4. TAXONOMY FOR APPLICATIONS}

Barriers: Lack of consistency in the classification of components, testing procedures, and interpretations of failure events. Approach: This roadmap will focus on developing taxonomy for applications in collaboration with stakeholders to provide consistency in classification and enable standardization of performance assessment of PHM. The objective is to develop and enable standard taxonomies covering $100 \%$ of critical equipment.

\begin{tabular}{|c|c|c|c|}
\hline & Roadmap Action Plan & Milestones & Targets/ Capabilities \\
\hline $\begin{array}{c}1-2 \\
\text { years }\end{array}$ & $\begin{array}{l}\text { Assumption: one company focus (initially) } \\
\text { - Compile differences between testing } \\
\text { and point of contacts } \\
\text { - Correlate for consistency } \\
\text { - Identify critical equipment/develop } \\
\text { critical assessment }\end{array}$ & $\begin{array}{l}\text { A "council" or other collaboration of the points of } \\
\text { contact } \\
\text { - Upper management agreement on approach }\end{array}$ & $\begin{array}{l}\text { Critical assessments of } \\
\text { inconsistencies in classifications } \\
\text { for PHM testing and } \\
\text { interpretation }\end{array}$ \\
\hline $\begin{array}{c}3-5 \\
\text { years }\end{array}$ & $\begin{array}{l}\text { - Identify common stakeholders } \\
\text { interpretations, jargon, language } \\
\text { - Develop "glossary" of failure-codes } \\
\text { and equipment classes } \\
\text { - Present/sell "glossary" }\end{array}$ & $\begin{array}{l}\text { - Standardized agreement from Council } \\
\text { - Implementation through CMMS } \\
\text { - Acceptance/buy-in of users }\end{array}$ & $\begin{array}{l}100 \% \text { critical equipment is } \\
\text { standardized }\end{array}$ \\
\hline $\begin{array}{c}5+ \\
\text { years }\end{array}$ & $\begin{array}{l}\text { Undertake continuous revision of } \\
\text { "glossary" as technologies emerge }\end{array}$ & $\begin{array}{l}\text { - Revisions by key points of contact } \\
\text { - Collaborative agreement on taxonomy }\end{array}$ & - $100 \%$ of compliance \\
\hline
\end{tabular}

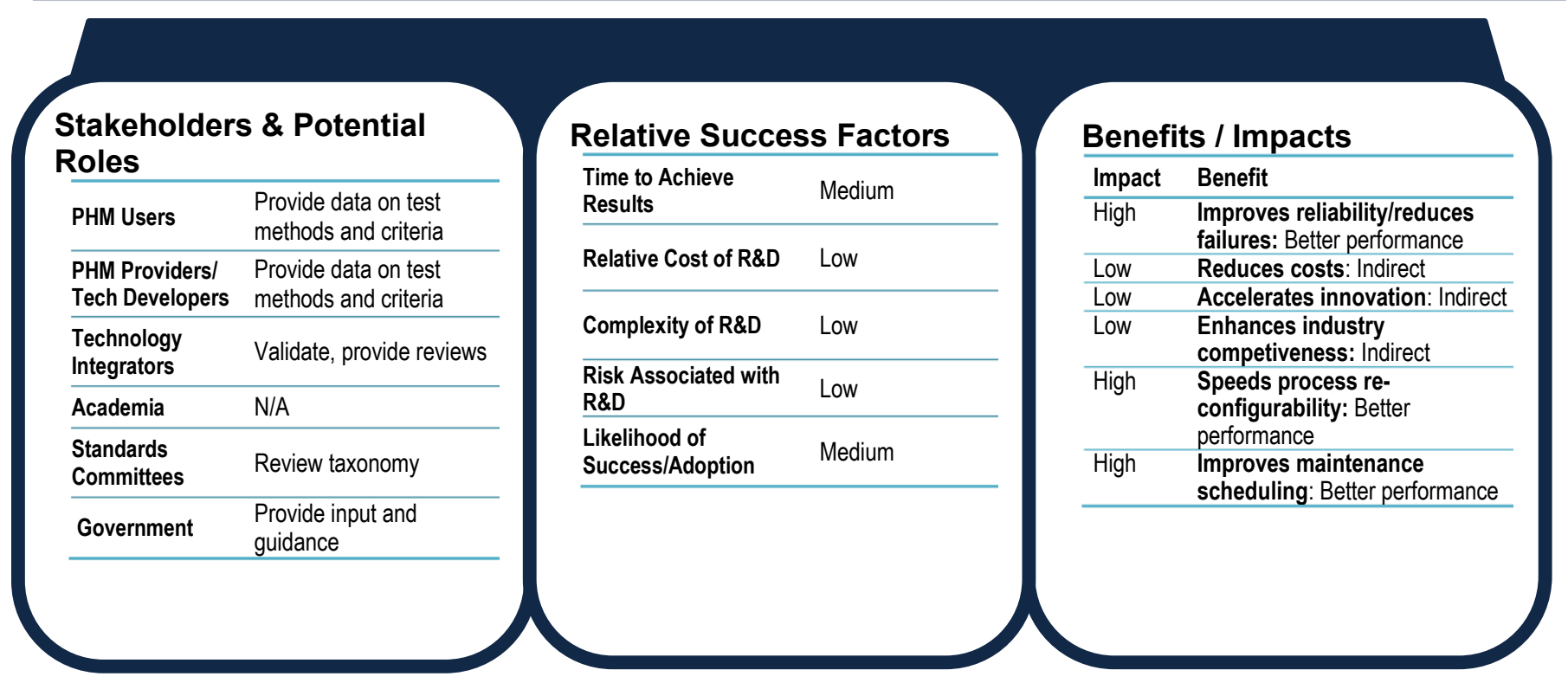




\section{Figure 3-5. Determination Of PHM DATA AND INFORMATION NeEdS}

Barriers: PHM solutions require tailoring and are not yet plug and play. User knowledge of systems (issues, failures, etc.) is lacking, and no standard method is available for calculating value and ROI. Event-driven data of good quality and integration of data (human actions) is lacking or insufficient; there is a general lack of knowledge of what is wanted from a PHM system.

Approach: The objective is to develop more or better standards for all aspects of the PHM process (data collection, data characterization, communication, integration, etc.). The approach includes standardization of record keeping and developing standard criteria for which applications or components/machines should be applied (prioritization criteria).

\begin{tabular}{|c|c|c|c|}
\hline & Roadmap Action Plan & Milestones & Targets/Capabilities \\
\hline $\begin{array}{c}1-2 \\
\text { years }\end{array}$ & $\begin{array}{l}\text { Collect information about the most } \\
\text { prevalent causes or triggers of downtime } \\
\text { Collect information about the existing data } \\
\text { collection; and characterization, } \\
\text { communication, etc. strategies } \\
\text { Determine most prevalent wants and } \\
\text { needs for a PHM system (such as } \\
\text { functions) }\end{array}$ & $\begin{array}{l}\text { - Collection of all needed information } \\
\text { - Repository of information established }\end{array}$ & $\begin{array}{l}\text { Survey of at least } 30 \text { different } \\
\text { organizations }\end{array}$ \\
\hline $\begin{array}{c}3-5 \\
\text { years }\end{array}$ & $\begin{array}{l}\text { - Define framework and key parameters } \\
\text { - Conduct sensitivity analysis } \\
\text { - Define inputs (extract features that best } \\
\text { inform PHM development) }\end{array}$ & - Framework for PHM aspects & $\begin{array}{l}\text { Framework that applies to } 75 \% \\
\text { of industry }\end{array}$ \\
\hline $\begin{array}{c}5+ \\
\text { years }\end{array}$ & - Develop pilot study & $\begin{array}{l}\text { - Completed pilot study } \\
\text { - Guidelines for PHM solutions }\end{array}$ & $\begin{array}{l}\text { - } 30 \% \text { reduction in development } \\
\text { and implementation time } \\
\text { - } 30 \% \text { increase in PHM adoption }\end{array}$ \\
\hline
\end{tabular}

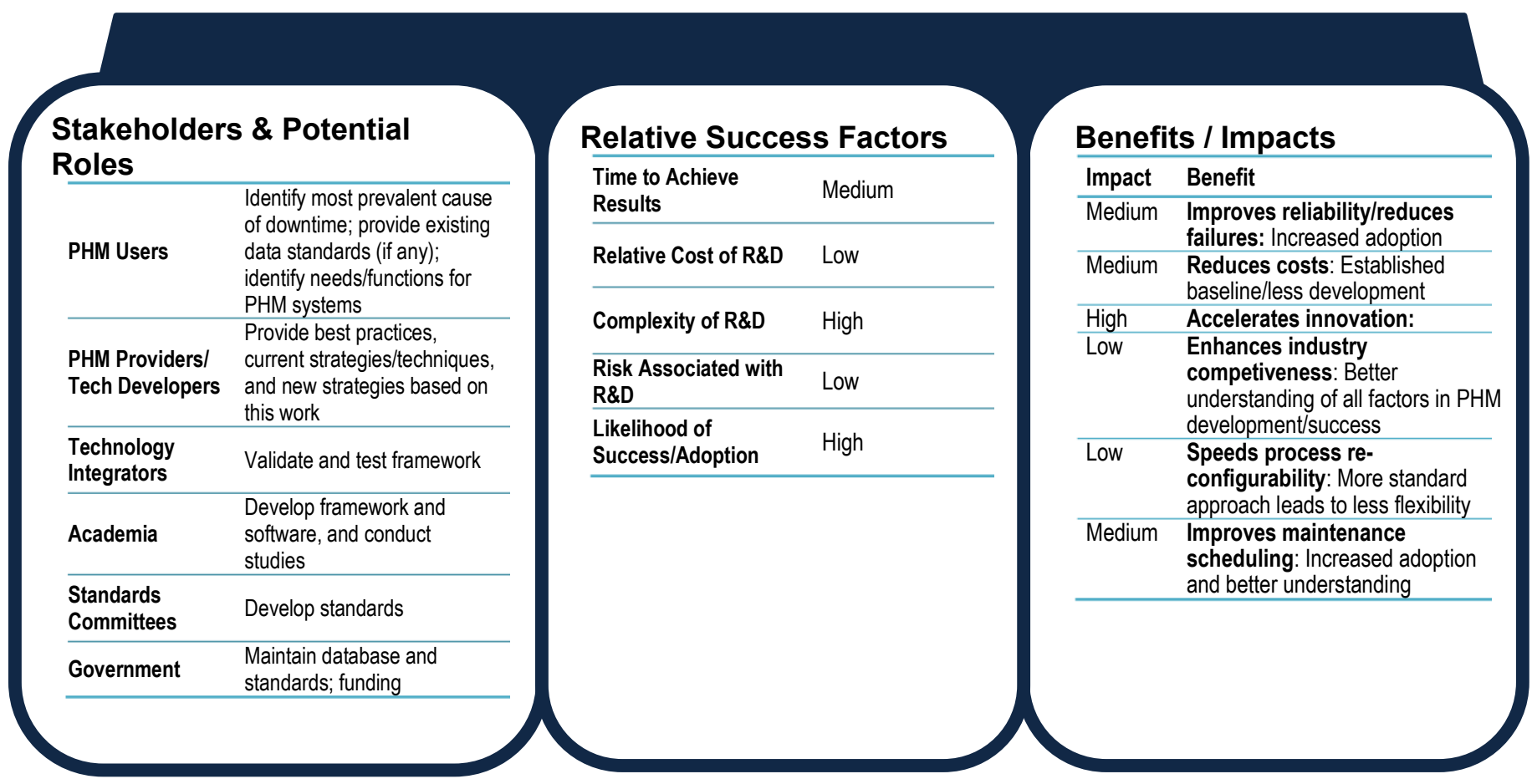




\section{FIgURE 3-6. FAILURE DATA FOR Prognostics and Diagnostics}

Barriers: Lack of good, accessible failure data for prognostics and diagnostics. Limited measurement and data collection methods and testbeds to provide data for PHM; lack of consistent data formats.

Approach: This project would develop methods and services to generate diagnostic and prognostic data sets for public consumption and validation and verification. Evidentiary testbeds would provide validation and data.

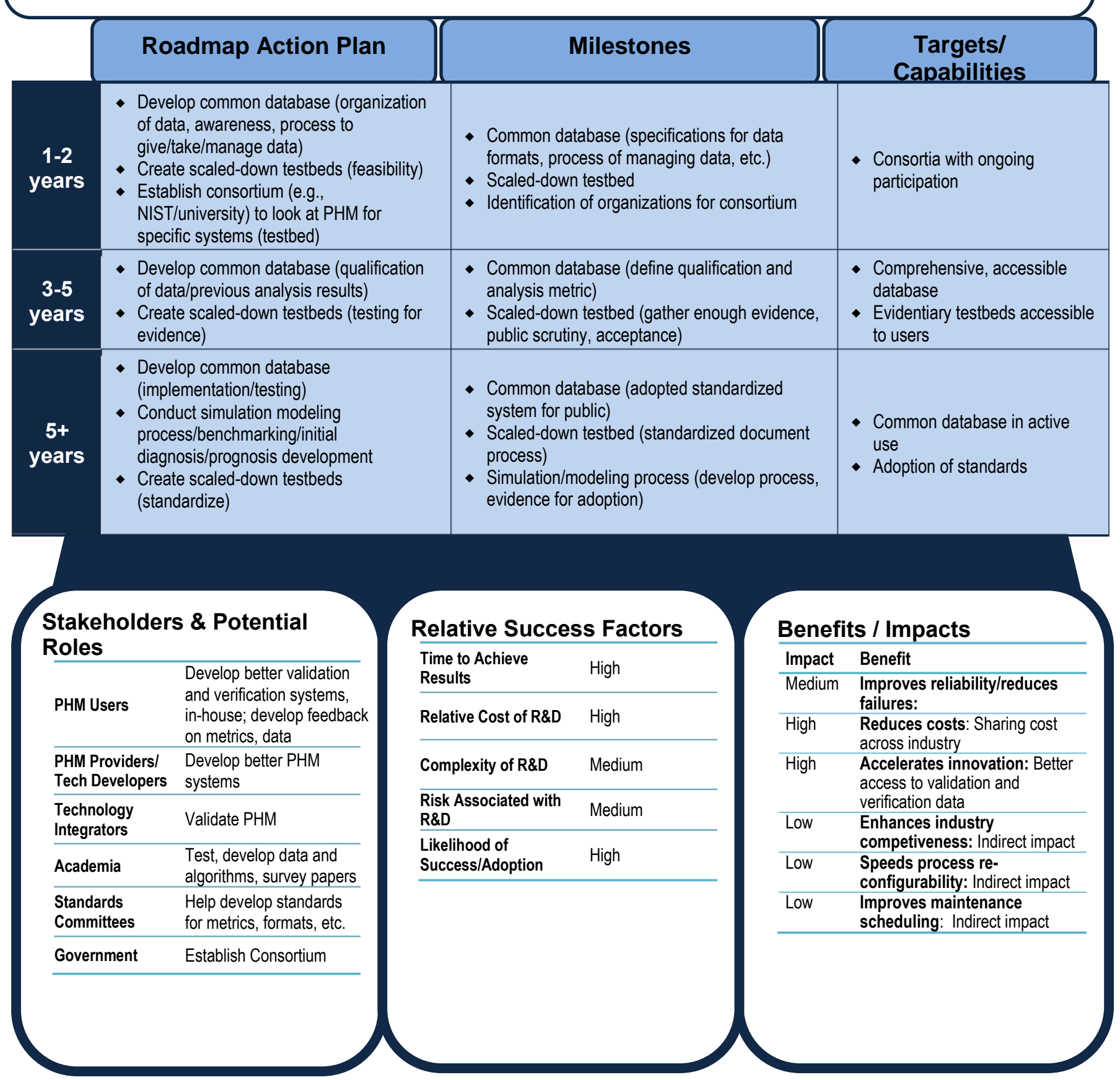




\section{PHM Infrastructure - Hardware, Software, and System Integration}

\subsection{Overview}

Section 4 presents the Roadmapping Workshop results of a breakout group focused on the topic of PHM Infrastructure - Hardware, Software, and System Integration. The section summarizes the discussions and activities conducted by this breakout group. Participants identified goals, desired capabilities, challenges and barriers, and priority roadmap topics. Some of the challenges identified extend beyond the scope of the PHM Infrastructure, such as the lack of a proven business case for PHM and the integration of PHM into business systems. A list of participants from all breakout groups is shown in Appendix A.

In recent years, there has been a concerted R\&D effort to develop PHM to augment smart manufacturing capabilities across many industries. Part of this effort has focused on improving the PHM building blocks - hardware, software, models and simulations (collectively known as the infrastructure) and the interplay between these fundamental parts. The advent of PHM capabilities has led to the realization of a number of sophisticated techniques and intelligent algorithms that can be applied to machinery data analysis, health assessment and decision-making. These enhanced capabilities have improved product quality, enabled the identification and performance of just-in-time maintenance, helped avoid catastrophic equipment failures and minimized equipment downtime.

While the current PHM state-of-the-art has enhanced the smart manufacturing mission, there are a variety of measurement science needs, challenges, and gaps that are hindering the widespread development and deployment of health monitoring, diagnostics, and prognostics technologies. Specific goals, capabilities, and challenges associated with PHM infrastructure development have been identified to advance this emerging space.

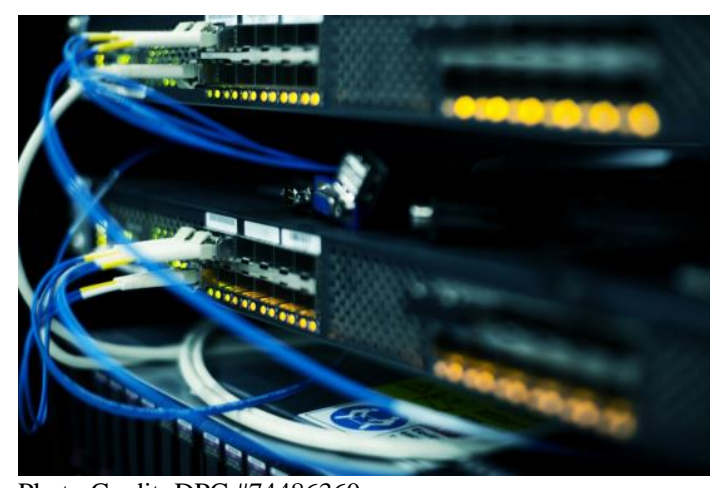

Photo Credit: DPC \#74486360

\subsection{Goals}

As PHM technologies continue to evolve, they provide richer and more relevant information for robust data-driven decision-making for improved performance, safety, reliability, and maintainability of instruments and equipment. These PHM technologies, in conjunction with smart manufacturing environments where cyber and physical systems are highly interconnected, are expected to improve the fidelity of manufacturing processes. Table 4-1 below highlights some high-level envisioned goals for PHM technology. These goals are separated into short, medium, and long timeframes as to when they are anticipated to be a commonplace. 


\begin{tabular}{|c|c|c|}
\hline \multicolumn{3}{|c|}{$\begin{array}{l}\text { Table 4-1. High-Level Goals for PHM } \\
\text { Hardware, Software, Integration, and Infrastructure }\end{array}$} \\
\hline $\begin{array}{l}\text { NEAR-TERM } \\
\text { (1 to } 2 \text { Years) }\end{array}$ & $\begin{array}{l}\text { MID-TERM } \\
\text { (3 to } 5 \text { Years) }\end{array}$ & $\begin{array}{l}\text { LONG-TERM } \\
(5+\text { Years })\end{array}$ \\
\hline $\begin{array}{l}\text { Maintenance } \\
\text { - Set of maintenance system } \\
\text { requirements } \\
\text { - Diagnosis certainty of } 65 \% \text {; } \\
\text { prognosis mean prediction certainty } \\
\text { of } 25 \% \\
\text { Data Management } \\
\text { - Machine tools with sensor data that } \\
\text { are accessible at sufficient sampling } \\
\text { frequency } \\
\text { - Established data exchange } \\
\text { standard (acquisition and archival) } \\
\text { - Standards for interoperability of } \\
\text { different data sources } \\
\text { - Open source or "maker-like" } \\
\text { movement for a common way to } \\
\text { 'speak' to sensors } \\
\text { Design } \\
\text { - PHM as a component or system } \\
\text { design attribute; similar to other } \\
\text { design aspects: weight, cost, } \\
\text { performance trade-offs }\end{array}$ & $\begin{array}{l}\text { Maintenance } \\
\text { - Diagnosis certainty of } 80 \% \text {; } \\
\text { prognosis mean prediction } \\
\text { certainty of } 50 \% \\
\text { - Machine/robot- auto-schedules/ } \\
\text { orders maintenance/service } \\
\text { - No periodic maintenance - only } \\
\text { maintenance done based on PHM } \\
\text { indications } \\
\text { - Predict with greater than } 90 \% \\
\text { confidence on critical failure } \\
\text { modes, with better than two } \\
\text { month horizon } \\
\text { - Monitoring of physical system on } \\
\text { a regular basis } \\
\text { Hardware } \\
\text { - Identification of sensors to } \\
\text { facilitate PHM for standard } \\
\text { manufacturing equipment } \\
\text { - Integration of PHM with intelligent } \\
\text { control of machines/processes } \\
\text { Software/ Tools } \\
\text { - Requirements for complete PHM } \\
\text { systems and architectures end-to- } \\
\text { end } \\
\text { - Physics-based simulation models } \\
\text { to enable "What-if" scenarios that } \\
\text { are verified with real systems }\end{array}$ & $\begin{array}{l}\text { Maintenance } \\
\text { - Diagnosis and prognosis certainty of } \\
\text { greater than } 95 \% \\
\text { - Level of interoperability so that } \\
\text { heterogeneous systems can respond to } \\
\text { impending failures } \\
\text { Data Management } \\
\text { - Requests for data security without } \\
\text { restricting data to those who need it } \\
\text { - Use of data as statistical input for } \\
\text { design tools to evaluate part cost, } \\
\text { "manufacturability" } \\
\text { - Six-Sigma probability of no false } \\
\text { positives } \\
\text { - Standards for interoperability of } \\
\text { different data sources } \\
\text { Infrastructure Security } \\
\text { - Creation of an evolving (adaptive) } \\
\text { security framework for PHM } \\
\text { Software/Tools } \\
\text { - Highly portable and easily configured } \\
\text { software prognostics tools } \\
\text { - Culture shift to enumerate/order } \\
\text { Computer Numerical Control (CNC) } \\
\text { features to facilitate PHM } \\
\text { measurements }\end{array}$ \\
\hline
\end{tabular}

\subsection{Desired Capabilities}

Researchers continue to build and improve on the functionalities of existing PHM systems all the while exploring what new capabilities PHM can perform. Table 4-2 highlights some specific desired capabilities for PHM systems and their importance in advancing PHM functionalities. The focus areas for this work span a number of areas.

\section{Hardware}

Enabling the use of PHM hardware and software in customized, agile, and flexible production lines is highly desirable. Embedded, self-powered PHM-related devices in-line with component manufacturing (sensors, corrosion detection, wire runs) will support this. Smart and/or self-aware and self-adapting sensing capabilities will be a key aspect. 


\section{Table 4-2. Desired Capabilities and Metrics for Infrastructure for PHM}

\section{Hardware}

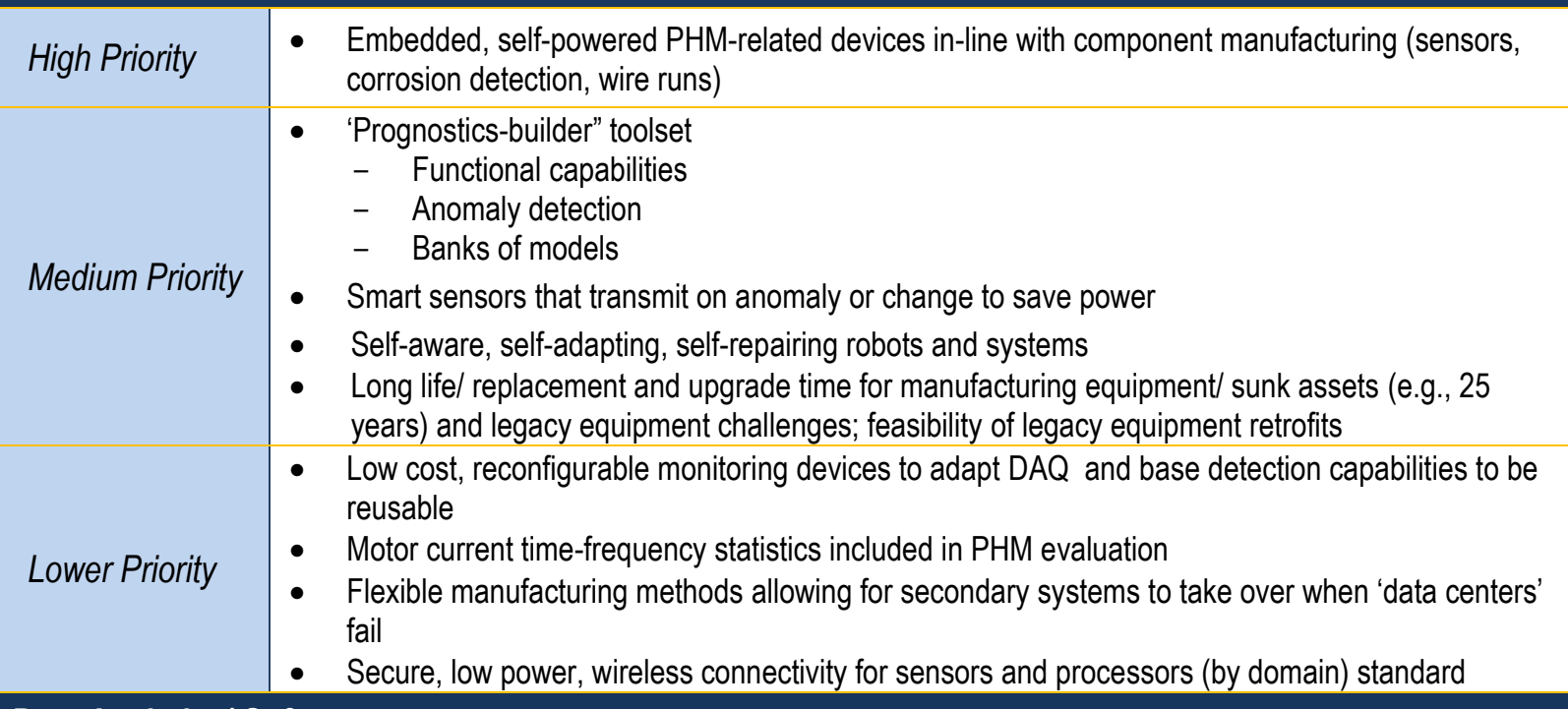

Data Analytics/ Software

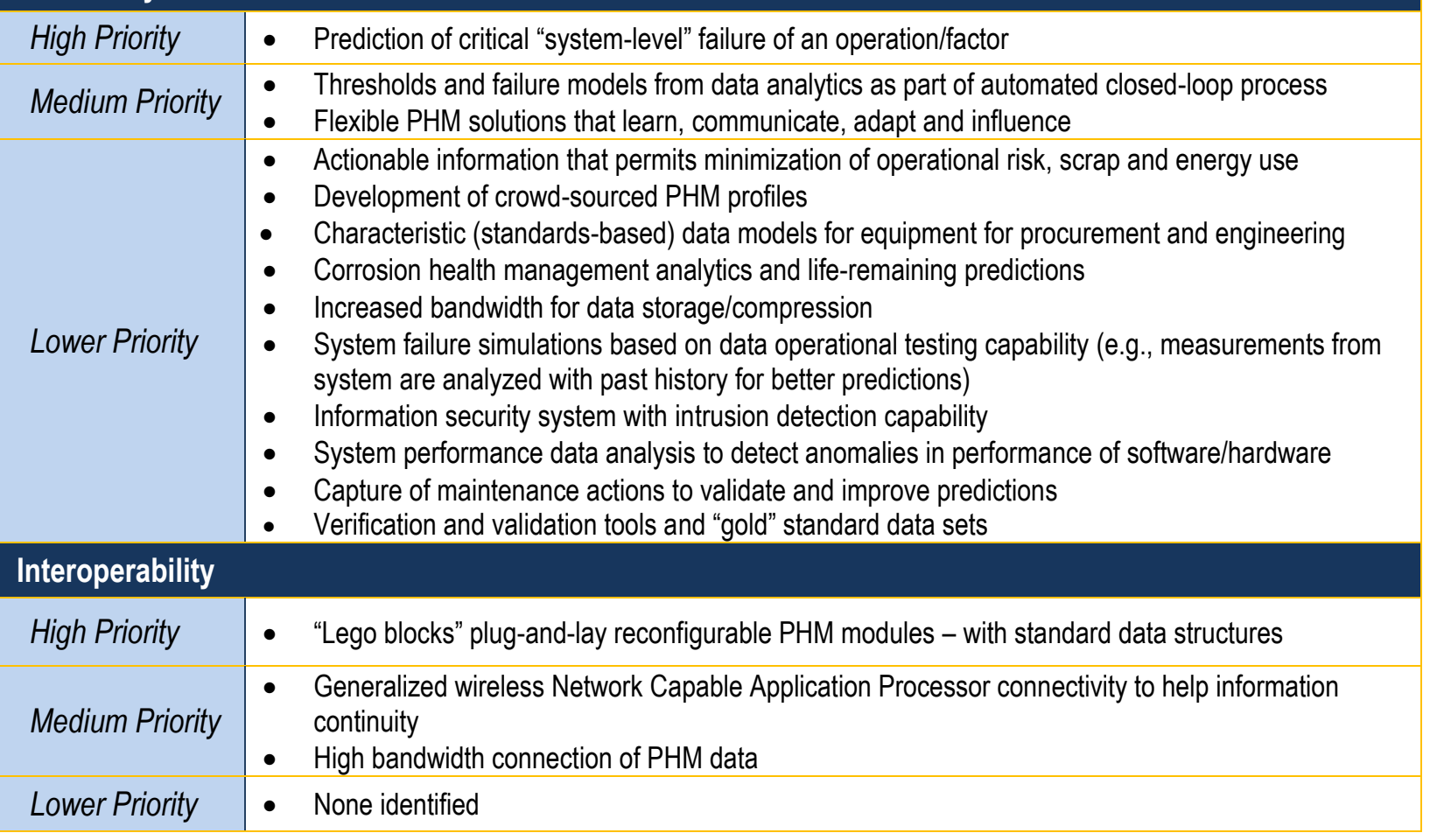

\section{Data Analytics/ Software}

A high priority is the ability to predict critical system level failures. This would require better, validated failure models based on data analytics along with verifiable failure thresholds. Capabilities are also needed to effectively integrate hardware and software with legacy systems and new complex, highly connected smart process/ manufacturing equipment. 


\section{Interoperability}

Better capabilities are needed to ensure seamless interconnectivity between PHM and internal and external communications gear - wireless networks, transponders and receivers, etc. This will require interoperability among a range of hardware. Plug-and-play, reconfigurable PHM modules that are standardized will support greater interoperability of interconnection of PHM, devices, and equipment across the plant, enabling more intelligent diagnostics and prognostics.

\subsection{Challenges and Barriers}

Specific desired capabilities and goals were identified for how PHM can contribute and advance the mission and objectives of many smart manufacturing initiatives. However, many of these cannot be realized yet due to a number of existing challenges and barriers that need to be addressed. Table 4-3 lays out a number of these barriers for PHM use and integration into modern manufacturing systems, particularly infrastructure. These challenges are grouped into four broad categories.

\section{Technology and Infrastructure Implementation}

This includes the issues arising from the development of hardware, software, model and simulations packages, and the associated integration with equipment. The key challenges center around security requirements for new systems that include wireless or other advances requiring increased surveillance; complexities of system integration across highly connected enterprises; and creating effective sensing with legacy equipment or equipment not designed for this capability. The long life of manufacturing equipment is another issue; improvements and replacements are slow to occur. Retrofits of advanced PHM on legacy equipment may not necessarily be affordable or practical. The same challenge is likely true for many smart manufacturing technologies.

\section{Economic and Business Requirements}

Issues arise due to the need to balance company operations versus business strategy. PHM is typically not well-integrated with equipment or process procurement requirements, and is also difficult to integrate with business systems. An underlying issue is the lack of a proven, readily justified business case for PHM based on a demonstrated value proposition. The availability of proven PHM software (with demonstrated cost benefits), for example, would help to establish a good business case. This is discussed in more detail in Chapter 5, Cross-cutting Challenges.

\section{Measurement and Standards}

The standards environment for PHM in smart manufacturing is challenging. PHM integration can be difficult because of too many standards; open standards are lacking or not sufficient. Many of the challenges identified focus on establishing baseline standards that the PHM industry can then design and build against. Particular areas where standards are lacking include software module standards and cybersecurity standards for robust, secure wireless sensors and networks.

In the measurement arena, there is uncertainty on what to measure or control via PHM to support consistent, reliable, and repeatable quality product. This is generally a process-specific challenge, i.e., better understanding of manufacturing processes is needed. However, there are common measurement requirements related to PHM that create challenges, such as defining usable metrics for PHM performance. 


\section{Human Elements}

The human element arises in business and manufacturing culture challenges. PHM development and implementation works best when championed or led by a management organization structure within an organization, i.e., a concerted effort toward PHM adoption. This requires the ability to articulate PHM needs and benefits throughout all levels of an organization.

For successful implementation, PHM information/data needs to be presented in a usable, actionable form to technicians and engineers - and they need to be trained how best to take action based on that information. Additional discussion on workforce issues is found in Chapter 5, Cross-cutting Challenges. Challenges inherently arise in the manufacturing environment where humans typically make these decisions (without automated systems) and are presented with new systems where these decisions are removed from operator control (issues of trust, reliability, etc.).

\begin{tabular}{|c|c|}
\hline \multicolumn{2}{|c|}{ Technology and Infrastructure Implementation } \\
\hline High Priority & $\begin{array}{l}\text { - Security concerns } \\
-\quad \text { PHM requires more data collection leading to increased surveillance/monitoring } \\
\text { - } \quad \text { Security concerns for wireless sensors } \\
\text { - } \quad \text { Lack of understanding PHM cyber-security challenges/guidelines }\end{array}$ \\
\hline Medium Priority & $\begin{array}{l}\text { - System integration is difficult - especially across an enterprise } \\
\text { - } \quad \text { Sensor placement due to equipment design limitations } \\
\text { - Lack of or insufficient existing machine data drives up cost of PHM due to the need to replicate } \\
\text { - } \quad \text { Immature models that are constantly evolving } \\
\text { - Lack of availability of generic PHM software solutions }\end{array}$ \\
\hline Lower Priority & $\begin{array}{l}\text { - Vast differences in scale and types of manufacturing } \\
\text { - } \quad \text { Prognostic metrics not universally agreed upon/available for validation } \\
\text { - } \quad \text { Lack of existing information/data infrastructure } \\
\text { - } \quad \text { Inability to provide actionable requirements to equipment suppliers by delivering the right data } \\
\text { - Limited PHM capabilities due to factory IT and legacy equipment (e.g., WIN 95/NT, etc.) } \\
\text { - Availability of port(s) for PHM connectivity } \\
\text { - } \quad \text { Non-invasive sensor data is only solution but there is a loss in process fidelity } \\
\text { - } \quad \text { Low-cost equipment limiting specific failure models for reliable RUL prediction }\end{array}$ \\
\hline \multicolumn{2}{|c|}{ Economic and Business Requirements } \\
\hline High Priority & $\begin{array}{l}\text { - Lack of PHM in procurement requirements and limited alignment with company/business } \\
\text { objectives } \\
\text { - } \quad \text { Difficulty integrating PHM with business systems (e.g., ERP) } \\
\text { - Lack of proven PHM software ( i.e., a credible/reliable "check engine light" ) }\end{array}$ \\
\hline
\end{tabular}




\section{Table 4-3. Barriers for PHM Infrastructure, Integration, and Utilization}

\begin{tabular}{|c|c|}
\hline Medium Priority & $\begin{array}{l}\text { - High costs of sensors, IT infrastructure, and data acquisition; added expense of PHM wire run } \\
\text { when capability does not have proven ROI } \\
\text { - Lack of funding for open source PHM software/sensor development and analysis } \\
\text { - Limited technology investment and maturation programs for PHM; finite capital investment } \\
\text { budgets with competing priorities } \\
\text { - Limited capital for non-recurring engineering for high fidelity, high performance PHM (i.e., lack of } \\
\text { inexpensive, good, fast PHM) } \\
\text { - Lack of plans for long-term ownership of data and analysis results (economics of data storage } \\
\text { and related issues) } \\
\text { - Limited credibility of the PHM system when in operation }\end{array}$ \\
\hline Lower Priority & $\begin{array}{l}\text { - Developing low cost PHM solutions - layers/non-embedded solutions } \\
\text { - Inability to ensure that PHM is a driver for component design } \\
\text { - Limited technology availability- large business can afford dedicated PHM group, small business } \\
\text { cannot }\end{array}$ \\
\hline \multicolumn{2}{|c|}{ Measurement and Standards } \\
\hline High Priority & $\begin{array}{l}\text { - Limited implementation of PHM when advanced by an open source community (creating } \\
\text { standards without royalties/free); lack of commitment to open standards }\end{array}$ \\
\hline Medium Priority & $\begin{array}{l}\text { - Limited software module standards } \\
\text { - Too many standards which limits integration of PHM (i.e., inability to coalesce on a smaller set of } \\
\text { key standards) } \\
\text { - Limited ability to address cybersecurity standards for robust, secure wireless sensors, networks } \\
\text { - Uncertainty on what to measure and/or control leading to consistent, reliable, repeatable quality } \\
\text { product } \\
\text { - Insufficient understanding of manufacturing processes, e.g. additive methods }\end{array}$ \\
\hline Lower Priority & $\begin{array}{l}\text { - Lack of standards/metrics for PHM system performance } \\
\text { - } \quad \text { Lack of policy standards } \\
\text { - } \text { Opused open database standards, such as those from the Machinery Information Management } \\
\text { - Lack of wireless hardware (MIMOSA) } \\
\text { - Limited diagnostics and prognostics of machinery standards (ISO 13373, under development) } \\
\text { - } \quad \text { Ability to measure ongoing process health, "how well are we doing" rather than just failure/ } \\
\text { impending failure } \\
\text { - Limited methods for feeding the results of PHM (data)back to system/component design } \\
\text { - Lack of a source/forum information exchange that lists all applicable standards } \\
\text { - No librarian/historian for national and ISO PHM standards reservoir }\end{array}$ \\
\hline \multicolumn{2}{|l|}{ Human Elements } \\
\hline High Priority & - None identified \\
\hline Medium Priority & $\begin{array}{l}\text { - Limited management structure within an organization for PHM development and implementation; } \\
\text { inability of company management organization structures to effectively understand/use PHM } \\
\text { - Ability to present PHM information/data in a usable, actionable form to technicians and engineers } \\
\text { - Articulating the PHM needs at high levels in any organization } \\
\text { - Recognition by designers of PHM solutions that systems will change }\end{array}$ \\
\hline Lower Priority & $\begin{array}{l}\text { - } \quad \text { Creating a paradigm shift in the middle management thought process when considering PHM } \\
\text { - } \quad \text { PHM systems that consider people, organizations, training and supply chain } \\
\text { - } \quad \text { Limited culture in OEMs for adoption of open source PHM (e.g., for information sharing) }\end{array}$ \\
\hline
\end{tabular}




\subsection{Priority Roadmap Topics}

Of the capabilities and challenges in Tables 4-2 and 4-3 that were identified as most important, the following four topics were put into priority roadmaps. Some of the high priority capabilities and challenges were combined and covered in the roadmaps.

- PHM as an Equipment Design Feature - In today's military platforms, reducing system size, weight, and power (SWaP) is critical for maximizing operational life and meeting budgetary constraints. ${ }^{5}$ Similarly, weight, cost and performance are often the key drivers that designers take into consideration when developing manufacturing systems. PHM is typically not thought of as a design feature due to limited awareness or unknown impact, but should be considered since a well-functioning PHM system can have bottom-line implications similar to other traditional ones like cost, weight, and performance.

- Open-Source Community for PHM - An open source development model provides for complete access to a product's design, and full redistribution of that design, including subsequent improvements to the design ${ }^{6,7}$. Such a model shares the risk and development capital expense across a number of institutions while enabling unrestricted collaborations and establishing some fundamental baseline designs and standards. This type of collaboration is extremely beneficial for problem-solving as well. Collaboration for PHM developments would establish a common baseline to start from and build upon.

- Embedded Sensors for PHM of Emerging Manufacturing Technologies - In general, when retrofitting currently-in-use equipment with value-added technologies such as PHM, there is a need to ensure that the technology installation process doesn't degrade/damage equipment performance. Attention is also needed during installation to ensure that the technology performs to specification. Identifying innovative ways to embed and operate PHM systems seamlessly should support optimal performance of the PHM and the production equipment.

- PHM Infrastructure to Deliver Relevant Timely Information - Accurate, timely, and actionable information has been a goal for manufacturers. However, when inaccurate data and/or incorrect information is delivered to the wrong operations level, actions are delayed and can, in many instances, lead to detrimental actions that damage or decommission instruments and equipment. Relevant, verified, and targeted PHM systems aim to deliver accurate status updates to the appropriately-targeted recipient.

\footnotetext{
${ }^{5}$ http://www.altera.com/end-markets/military-aerospace/swap/mil-swap.html

${ }^{6}$ Lakhani, K.R.; von Hippel, E. (June 2003). "How Open Source Software Works: Free User to User Assistance". Research Policy 32 (6): 923-943. doi:10.1016/S0048-7333(02)00095-1.

${ }^{7}$ Gerber, A.; Molefo, O.; Van der Merwe, A. (2010). "Documenting open-source migration processes for re-use". In Kotze, P.; Gerber, A.; van der Merwe, A. et al. Proceedings of the SAICSIT 2010 Conference - Fountains of Computing Research. ACM Press. pp. 75-85. doi:10.1145/1899503.1899512. ISBN 978-1-60558-950-3
} 


\section{Figure 4-1. PHM as an EquiPMENT Design FEATURE}

Barriers: Limited consideration of PHM as a design attribute during the many trade-offs made in a system and/or component design process - not viewed the same as weight, cost, performance, etc.

Approach: The approach includes conducting activities to support considerations of PHM in design, and to affect changes in current paradigms used during a platform, system, and/or component design definition process. This paradigm shift would enable the use of PHM capabilities and their information products to change design thinking to save weight, costs, redundancy, and increase performance.

\begin{tabular}{|c|c|c|c|}
\hline & Roadmap Action Plan & Milestones & Targets/ Capabilities \\
\hline $\begin{array}{c}1-2 \\
\text { years }\end{array}$ & $\begin{array}{l}\text { Study what is feasible in this area } \\
\text { Focus on component weight reduction and cost } \\
\text { savings associated with excess material weight } \\
\text { and redundancies of component elements like, } \\
\text { actuators, engine disks } \\
\text { Generate a focused demonstration project plan }\end{array}$ & $\begin{array}{l}\text { Identification of feasible PHM design } \\
\text { features } \\
\text { - Fully-scoped demonstration program } \\
\text { for a set of PHM design features }\end{array}$ & $\begin{array}{l}\text { - Early demonstration of PHM } \\
\text { into key design features }\end{array}$ \\
\hline $\begin{array}{c}3-5 \\
\text { years }\end{array}$ & $\begin{array}{l}\text { Conduct one or more demonstration programs to } \\
\text { show PHM can be integrated in design with little or } \\
\text { no impact on structure or component safety } \\
\text { Expand scope of this application to include } \\
\text { additional high value components } \\
\text { Start multiple demonstrations to provide the } \\
\text { component designer the evidence that the } \\
\text { tradeoffs are safety and confidence }\end{array}$ & $\begin{array}{l}\text { - Initial pilot demonstrations completed } \\
\text { Project plan completed for additional } \\
\text { demonstrations with defined } \\
\text { components and schedule } \\
\text { - Multiple-equipment/component } \\
\text { demonstrations initiated }\end{array}$ & $\begin{array}{l}\text { Demonstration of practical } \\
\text { PHM value proposition for each } \\
\text { design }\end{array}$ \\
\hline $\begin{array}{c}5+ \\
\text { years }\end{array}$ & $\begin{array}{l}\text { - Continue multiple demonstration and test cases } \\
\text { - Get OEM and industry buy-in }\end{array}$ & $\begin{array}{l}\text { - Demonstrations completed } \\
\text { - Test cases/best practices published }\end{array}$ & $\begin{array}{l}\text { - PHM adopted in multiple } \\
\text { equipment sectors }\end{array}$ \\
\hline
\end{tabular}

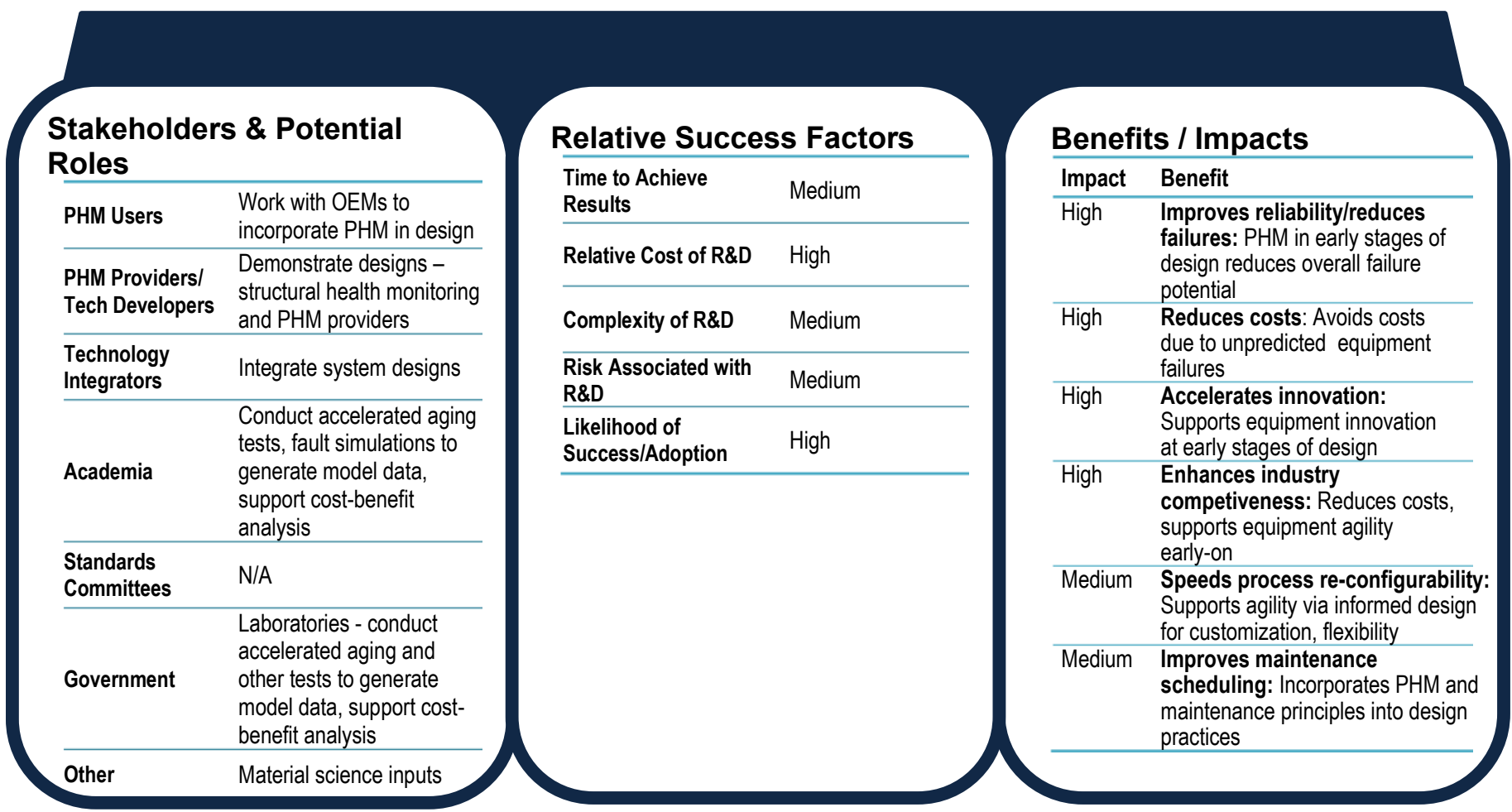




\section{Figure 4-2. OPEN SOURCE COMMUNITY FOR PHM}

Barriers: Cost and complexity to implement prognostics and PHM on new equipment is high.

Approach: The objective is to develop an open source architecture, which will reduce costs and complexity for implementation and provide the foundation for an end-to-end PHM architecture. The approach includes collection of data, identification of systems and devices that should be covered, and development of an open community framework and architecture.

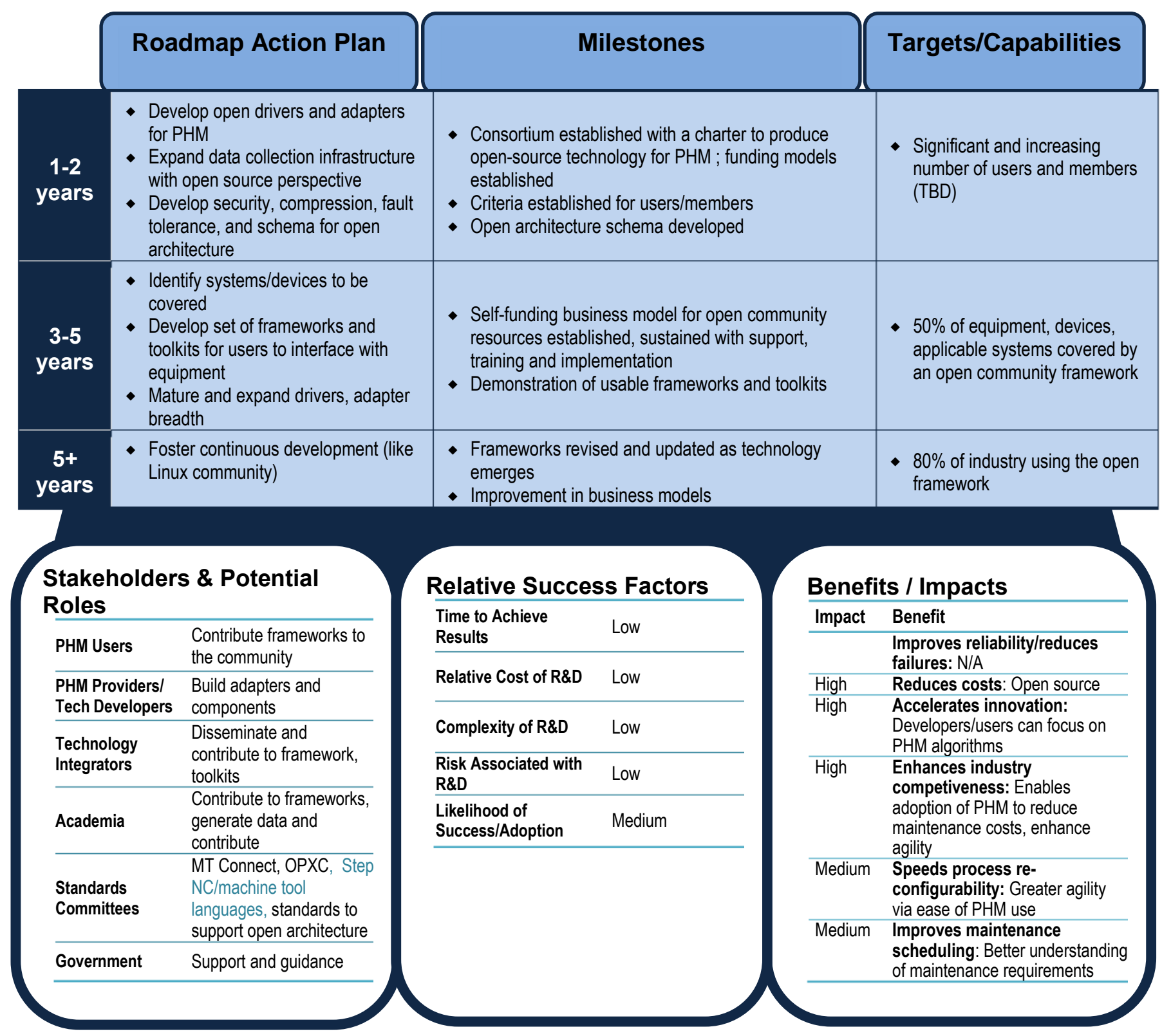




\section{Figure 4-3. EMBEDded SENSORS FOR PHM OF EMERging MANUFACTURING TECHNOLOGIES}

Barriers: Utilizing embedded PHM sensors (in structure or surface mounted) wire runs, self-powering technology and other related devices during the manufacturing process for structures (structural components) is promising but challenging to implement due to complexity and cost, and possible impacts on structural integrity.

Approach: This effort will study the feasibility of embedded sensor and technology concepts for PHM, based on new and/or evolving manufacturing technologies like 3D printing; determine the sensors, wire runs, and devices that could be of most value.

\begin{tabular}{|c|c|c|c|}
\hline & Roadmap Action Plan & Milestones & Targets/ Capabilities \\
\hline $\begin{array}{c}1-2 \\
\text { years }\end{array}$ & $\begin{array}{l}\text { - Conduct technology feasibility study } \\
\text { Define which devices/sensors are suitable for } \\
\text { embedded PHM } \\
\text { - Assess impact on structural integrity } \\
\text { - Map to new or existing manufacturing } \\
\text { processes }\end{array}$ & $\begin{array}{l}\text { Sensor and structure components for } \\
\text { demonstration identified } \\
\text { Candidate manufacturing technology } \\
\text { process identified for demonstration }\end{array}$ & $\begin{array}{l}\text { Feasible and achievable cost } \\
\text { targets }\end{array}$ \\
\hline $\begin{array}{c}3-5 \\
\text { years }\end{array}$ & $\begin{array}{l}\text { - Conduct manufacturing demonstration with } \\
\text { embedded PHM; include manufacturing } \\
\text { processing capability } \\
\text { - Generate architecture definition } \\
\text { - Develop notional design integration and } \\
\text { requirements }\end{array}$ & $\begin{array}{l}\text { - Technology demonstration and report } \\
\text { - Architecture for sensor and structure } \\
\text { integration } \\
\text { - Recommended design integration and } \\
\text { requirements report }\end{array}$ & $\begin{array}{l}\text { No degradation in structure and } \\
\text { component reliability }\end{array}$ \\
\hline $\begin{array}{c}5+ \\
\text { years }\end{array}$ & $\begin{array}{l}\text { Integrate with actual manufacturing production } \\
\text { - Expand scope to include other sensor types and } \\
\text { components for manufacturing technologies } \\
\text { Additional demonstration projects for emerging } \\
\text { technologies and for validation }\end{array}$ & $\begin{array}{l}\text { Results of manufacturing process } \\
\text { capabilities study } \\
\text { - Long term validation and verification } \\
\text { assessments }\end{array}$ & - High component reliability \\
\hline
\end{tabular}

\section{Stakeholders \& Potential}

\section{Roles}

\begin{tabular}{ll}
\hline PHM Users & $\begin{array}{l}\text { Test/demonstrations of } \\
\text { sensors }\end{array}$ \\
\hline $\begin{array}{l}\text { PHM Providers/ } \\
\text { Tech Developers }\end{array}$ & $\begin{array}{l}\text { Test/demonstrations of } \\
\text { sensors }\end{array}$ \\
\hline $\begin{array}{l}\text { Technology } \\
\text { Integrators }\end{array}$ & $\begin{array}{l}\text { Component manufacturer } \\
\text { contributes to } \\
\text { test/demonstrations }\end{array}$ \\
\hline Academia & $\begin{array}{l}\text { Material science } \\
\text { assessment }\end{array}$ \\
\hline Standards & Testing standards \\
Committees & Regulation of structural \\
\hline Government & life and funding \\
\hline Other & OEM involvement \\
\hline
\end{tabular}

\section{Relative Success Factors} Time to Achieve Medium

Results

Relative Cost of R\&D Medium

Complexity of R\&D Medium

Risk Associated with Medium

R\&D

Likelihood of

Success/Adoption

High

\section{Benefits / Impacts}

Impact Benefit

High Improves reliability/reduces failures: Sensor data enhances failure prediction/ prevention

High Reduces costs: Reduces failure incidence and related costs

High Accelerates innovation: Supports new technology adoption and agility/flexibility

High Enhances industry competiveness: Reduces costs due to failure

High Speeds process reconfigurability: Enables better response to process/product changes

Medium Improves maintenance scheduling: Validation for maintenance scheduling 


\section{Figure 4-4. PHM INFRASTRUCtuRE to Deliver ReleVANT, TIMELY INFORMATION}

Barriers: Inability to make good decisions based on data; wrong information and detail at the wrong level.

Approach: The approach involves developing the appropriate information at each level to facilitate key operations and maintenance decisions. The outcome is a traffic light approach to data (green, yellow, red) for actionable decisions.

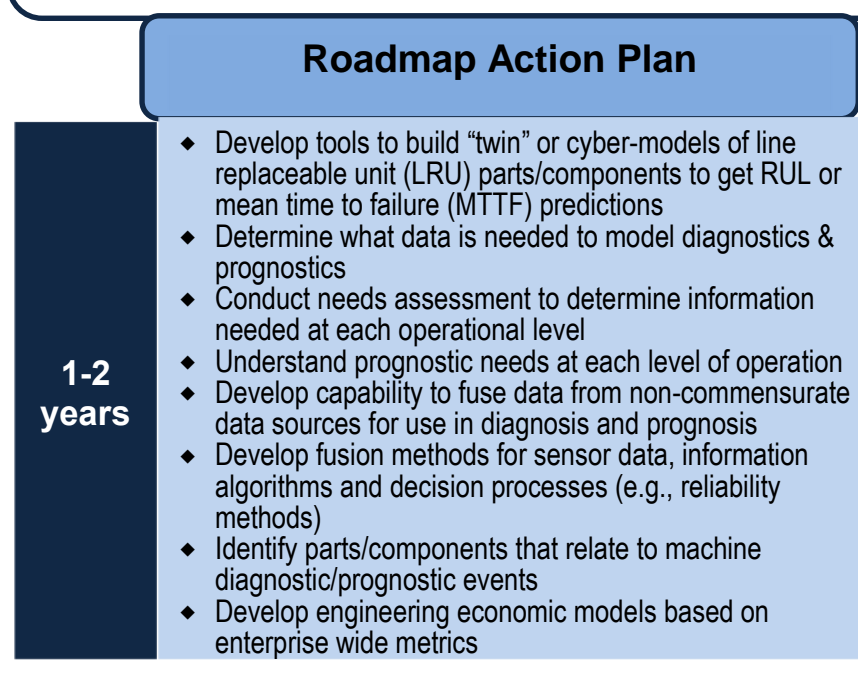

- Develop cloud-based data repository and analytic engine to refine decision-making

- Understand gaps in diagnostic and prognostic algorithms for each level of operation

- Develop tools for component "twin" cyber models for LRU predictions

- Develop technology for adaptable alarms based on condition

- Develop models to interface with enterprise systems

- Continue methods and other development

- Develop advanced usage-based models to construct

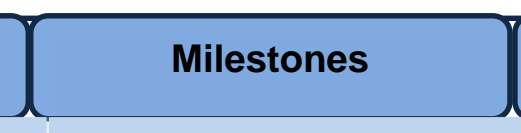

- High fidelity analytic models and required data sets assembled, verified and validated for selected machines

- Operational level decision needs established

- Demonstrated ability to make a limited set of decisions based on disparate data

- Demonstrated ability to identify specific degraded components

- Demonstration that PHM system can provide appropriate direction to enterprise resource planning (ERP) systems

- Demonstrated limited set of decisions made or refined from cloud-based data

- Identification of specific prognostic needs based on ability to make accurate decisions

- Demonstrated "twin" cyber models for selected machines

- Demonstrated alarm reconfiguration usage conditions

- Expansion of data sets demonstrated in 1-2 year milestones

- Validated usage models based on
Targets/ Capabilities

- Models for 5 major manufacturing equipment demonstrated, e.g., 5 types of machine tools

- Demonstrated fusion of 5 to 6 data sources

- Decision accuracy improved by PHM by $50 \%$ based on model based simulation

- Proven 50\% improvement in alarm accuracy

- Increased decision making

Stakeholders \& Potential
Roles
real world experience and data
Relative Success Factors

Time to Achieve Results

Relative Cost of R\&D Medium

Complexity of R\&D Medium

Risk Associated with R\&D

Likelihood of Success/Adoption

Low

High

-

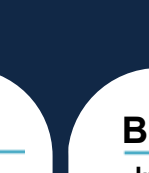

\begin{tabular}{ll} 
Benefits / Impacts \\
\hline Impact & Benefit \\
\hline High & $\begin{array}{l}\text { Improves reliability/reduces } \\
\text { failures: Better data for prevention }\end{array}$ \\
\hline High & Reduces costs: Reduced failures \\
\hline Medium & $\begin{array}{l}\text { Accelerates innovation: } \\
\text { Innovations in PHM methods }\end{array}$ \\
\hline Medium & $\begin{array}{l}\text { Enhances industry } \\
\text { competiveness: Reduces costs, } \\
\text { provides agility }\end{array}$ \\
\hline High & $\begin{array}{l}\text { Speeds process re- } \\
\text { configurability: The right data is } \\
\text { used to make quick decisions }\end{array}$ \\
\hline High & $\begin{array}{l}\text { Improves maintenance } \\
\text { scheduling: Improved decision } \\
\text { making in real-time }\end{array}$ \\
\hline
\end{tabular}




\section{Grosscutting Ghallenges}

There are a number of challenges that cut across the entire spectrum of PHM topics. These include:

\section{Data Collection and Extraction of Information}

Challenges exist for data collection and extraction of useful information from data (e.g., data quality, utility, and availability at the levels required), especially to generate timely, actionable intelligence with which to make appropriate decisions. Data collection is impeded by a lack of sensors and measurement methods for efficient and effective data collection that is tailored for the use of effective PHM for smart manufacturing systems. In addition, data and data standards inconsistencies make it difficult to uniformly apply PHM across the diverse spectrum of manufacturing equipment and systems; standardized data formats and taxonomies could help to encourage the use and improve the effectiveness of PHM. The best data collection comes from opportunistic failures. However, it is not practical to force machines/systems into a fault/failure state for the sole purpose of acquiring good PHM data. An ongoing challenge is how to generate accurate data for PHM without damaging equipment or impacting productivity.

\section{Models, Simulation, and Visualization (MSV)}

An overarching challenge is the limited availability of models to support PHM with validated accuracy and predictive capabilities. MSV is hampered by the wide variety of systems and equipment, the lack of integration of legacy systems (that are now becoming interconnected), and data availability. The lack of consistent, high quality data exacerbates modeling limitations; historical and/or practical/operating data is essential for model validation and accuracy.

In addition to supporting predictive maintenance and system performance, models are needed to help demonstrate the value of PHM and its use and performance in practice. It is difficult to build a business case for PHM without models that can reliably predict performance and other improvements (and their cost and other positive impacts).

\section{Design Considerations}

There are potential high-impact opportunities for improving the performance and maintainability of equipment and processes by incorporating PHM at the early design stage. Current equipment design philosophies do not adequately consider PHM and/or how it [PHM] could change over time. Designing a systems approach for PHM, such as that needed for smart manufacturing systems, is not effectively incorporated in today's design practices. A concerted effort involving both users and OEMs might be needed to effectively integrate PHM at the equipment and systems design levels.

\section{Workforce and Training}

PHM has been in use for many decades. Newly emerging PHM tools and techniques for smart manufacturing are incorporating a greater level of sophistication, modern information technology (IT), and networks that are highly connected and span the entire manufacturing enterprise. To operate effectively in the smart manufacturing environment, the next generation workforce will need new skills and training that are currently lacking (e.g., information-based decision making). Filling this gap will require a mix of technical vocational educational opportunities, new community college programs, technical training, and changes in engineering/science curricula in the higher education system. Currently, 
there is a lack of human talent and skilled professionals trained by accredited institutions. Even with systems that are highly automated, domain experts will still be needed, who will require continuous training as technologies emerge.

Currently, PHM-specific majors and curricula are lacking in institutions of higher education. Majors should not be limited to Electrical Engineering, but should be inter-disciplinary (e.g., mechanical/ industrial engineering, systems engineering, business, information technology, etc.). Post-education, there is a lack of engineers qualified or experienced with PHM analytics.

\section{Human Factors}

Human and cultural elements will come into play as more sophisticated PHM techniques emerge for the plant floor. Veteran operators are less likely to accept PHM technologies that make decisions based on machine thinking, especially if these same human operators have been responsible for recommending courses of actions (prior to the introduction of any PHM technologies). There may be resistance to the idea of machines making decisions with minimal human interaction, unless the value and reliability has been fully demonstrated. Keeping humans in the loop (and when to do so) will be a significant consideration. Non-automated, manual (human) recording of data, maintenance events, and failures could continue to limit development and validation of PHM systems.

Existing PHM techniques may be outdated and unable to take advantage of the full opportunity of smart manufacturing environments. Although PHM has been in use in some form for years, data-driven decision-making is not sufficiently embedded in today's manufacturing culture and environment. One of the challenges is changing the operators' perception so they trust data enough to act on it. In some cases, operators' fear of being constantly monitored may be a factor in adoption.

Operators on the front line of manufacturing decision-making will need to understand how advanced PHM can provide increased value over traditional methods in this highly-connected environment. Demonstrating how advanced PHM can be integrated with intelligent equipment and processes to make their jobs easier and more productive could go a long way toward fostering acceptance and cultural change.

\section{Business Case for PHM}

A strong business case is necessary for accelerating the development and adoption of PHM for smart manufacturing. Business models and a strong value proposition for PHM are currently lacking. This represents a priority challenge, as the business case directly impacts the ability to sell PHM to decisionmakers. Management support for PHM investments (R\&D or technology) can be lacking and/or competing with other manufacturing priorities where the cost-benefits are more certain.

Reliable, credible methods for quantifying ROI are not readily available or well-justified. Good predictive models, data, methods, and metrics for measuring PHM performance are all key factors for building the business case - and limitations exist in all these areas (see also Figures 3.2 and 3.3). Demonstrating the value of PHM in practice can be challenging, since it often must be measured over equipment life and maintenance cycles. The cost of enabling technology and infrastructure can also appear high when compared with benefits. Today, there is no standard way to quantify ROI related to developing or implementing PHM. This includes quantifying the associated costs of sensors, IT equipment, data acquisition, and maintenance. There is a strong need to develop a universal framework for cost-benefit analysis of PHM in manufacturing, and to clearly demonstrate/assess the benefits of various components and infrastructure. This would potentially accelerate greater investment and adoption. 


\section{Next Steps}

It is anticipated that this report will be used by the stakeholder community to guide future directions for the development of new technologies and infrastructure to support widespread use of PHM in smart manufacturing environments. Those organizations involved in conducting PHM-related R\&D, developing new products and services, and directly involved with implementation of PHM systems are likely to find this report useful.

NIST is carefully reviewing the workshop findings to update the PHM4SMS project's research direction to further align it with industry's needs and priorities. The PHM4SMS project team is currently focused on three specific efforts that will be informed by the key findings highlighted in the priority roadmaps presented in this document. No single roadmap stands out as being entirely synchronized with the forthcoming research efforts; rather, numerous roadmap themes are echoed. Some of these themes include a focus on sensing, data collection and organization, consistent metrics and standards, and promotion of community-wide PHM capabilities. The roadmaps most relevant to these research efforts include:

- Breakout Topic: PHM Manufacturing Process Techniques and Metrics

- Advanced Sensors for PHM in Smart Manufacturing

- PHM Data Format, Taxonomy, and Architecture

- Breakout Topic: PHM Performance Assessment

- Overarching Architecture Framework for PHM with Standards and KPIs

- Identification of PHM Performance Metrics

- Failure Data for Prognostics and Diagnostics

- Breakout Topic: PHM Infrastructure - Hardware, Software, and Integration

- Open-Source Community for PHM

- PHM Infrastructure to Deliver Relevant Timely Information

Collectively, the three research efforts described below are expected to address numerous elements of these roadmaps in their long-term plans. Table 6-1 shows how the aforementioned priority roadmaps relate to the three NIST PHM4SMS research efforts. Since these research efforts are still relatively young, numerous details will be refined as these efforts begin to mature.

Table 6-1 - Select Priority Roadmaps and their Relationship to NIST Research

\begin{tabular}{|l|c|c|c|}
\hline \multicolumn{1}{|c|}{ PRIORITY ROADMAPS } & \multicolumn{2}{c|}{ NIST RESEARCH EFFORTS } \\
\cline { 2 - 4 } & $\begin{array}{c}\text { Machine Tool } \\
\text { Linear Axes }\end{array}$ & $\begin{array}{c}\text { Systems-Level } \\
\text { Diagnostics and } \\
\text { Prognostics }\end{array}$ & PHM4Robotics \\
\hline Topic: PHM Manufacturing Process Techniques and Metrics & $\checkmark$ & $\checkmark$ & $\checkmark$ \\
\hline Advanced Sensors for PHM & & $\checkmark$ & $\checkmark$ \\
\hline PHM Data Format, Taxonomy, and Architecture & & $\checkmark$ & $\checkmark$ \\
\hline Topic: PHM Performance Assessment & & $\checkmark$ & $\checkmark$ \\
\hline Overarching Architecture Framework for PHM with Standards \& KPIs & & $\checkmark$ & $\checkmark$ \\
\hline Identification of PHM Performance Metrics & $\checkmark$ & & \\
\hline Failure Data for Prognostics and Diagnostics & & $\checkmark$ & $\checkmark$ \\
\hline Topic: PHM Infrastructure - Hardware, Software, and Integration & $\checkmark$ & $\checkmark$ & $\checkmark$ \\
\hline Open-Source Community for PHM & $\checkmark$ & $\checkmark$ & $\checkmark$ \\
\hline PHM Infrastructure to Deliver Relevant Timely Information & & $\checkmark$ \\
\hline
\end{tabular}


It is important to note that each research effort is likely to touch the identified priority roadmaps in unique ways, at different time horizons, and at varying levels of intensity. Some similarities are also expected, as well. For example, each of the NIST research efforts is expected to leverage the Advanced Sensors for PHM roadmap which is going to include taking inventory of relevant sensors. There are likely to be overlapping sensors identified (e.g., sensors capable of measuring positional accuracy would be relevant to all three research efforts or sensors capable of measuring motor current), yet there will be differences. One example is that the breadth of identified sensors will be different; this is apparent in the componentlevel research of the Machine Tool Linear Axes Test bed as compared to the systems-level test bed encompassing numerous machine tools.

\subsection{Machine Tool Linear Axes Diagnostics}

A linear axis is a vital subsystem of machine tools, which are often critical systems within many manufacturing operations. When installed and operating within a manufacturing facility, a machine tool needs to stay online and in good condition for parts production. All machine tools degrade during operations, yet knowledge of that degradation is elusive; specifically, accurately detecting degradation of linear axes is a manual and time-consuming process. Thus, manufacturers need quick and automated methods for continual diagnosis of machine tool linear axes without disruptions to production.

This effort is focused on developing a sensor-based method to quickly estimate the degradation of linear axes and is supported by the development of a linear axes test bed, as depicted in Figure 6-1. This method leverages data collected from a NIST-developed 'sensor box' to detect translational and angular changes due to axis degradation, and the test bed allows assessment of the new method against a conventional laser-based system. Simulations revealed that the multi-sensor-based method is capable of achieving test uncertainty ratios $\left(\mathrm{TURs}^{8}\right.$ ) of at least $4: 1$.

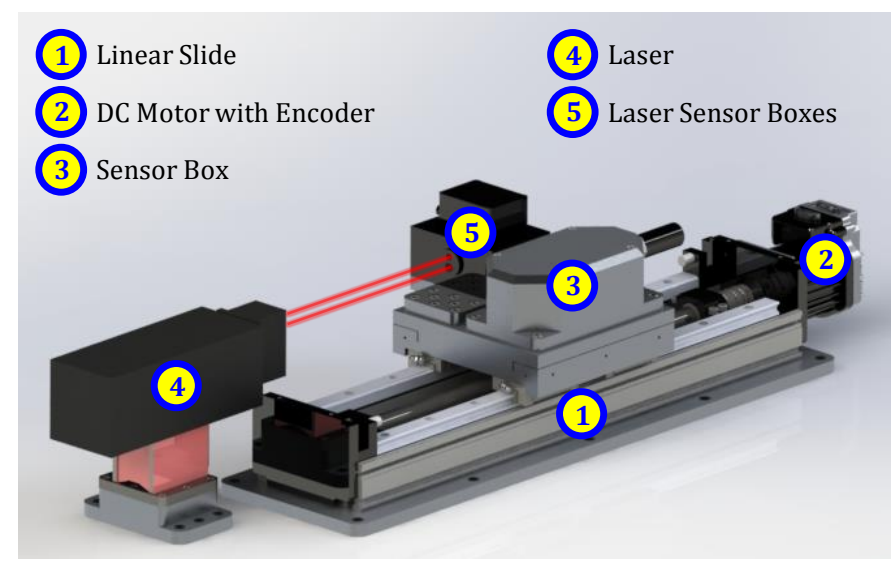

Figure 6-1 - Rendered image of linear axis testbed for testing of sensor-based method.

Once the method is verified for diagnostics of linear axes, further tests may show the value of certain metrics for prognostic purposes to estimate the remaining useful life (RUL). If the data collection and analysis is integrated within a machine controller, the process may be seamless. Data can be periodically collected to enable diagnostics and prognostics of linear axes for optimization of maintenance scheduling

\footnotetext{
${ }^{8}$ The test uncertainty ratio (TUR) is the ratio of the tolerance to the uncertainty of the measurement. Typically, a TUR of at least 4:1 is required; the larger, the better for a measurement system.
} 
and part quality. Therefore, the method is expected to result in reduced production losses for a machine tool.

This method will also enable verification and validation of other (built-in or otherwise) PHM techniques that aim to characterize translation and rotational errors and degradation. Likewise, this method will produce reference data sets that can be used by PHM developers as test data so they do not have to risk damaging their own equipment or impacting their productivity. This method will ultimately lead to standards to measure and predict linear axes degradation.

\subsection{Systems-Level Monitoring, Diagnostics, and Prognostics}

The health and status of equipment on the shop floor can have far-reaching effects on the overall operations of a manufacturing facility. In fact, many of the inefficiencies in manufacturing, such as those created by machine degradation and failure, occur at the systems level. Understanding and responding to these inefficiencies requires manufacturers to collect and assess the most appropriate data to forecast faults and failures accurately and efficiently with no disruption to operations. However, systems are inherently complex and critical components often fail to communicate with each other effectively even if their operations are intimately connected. Other challenges also exist that prevent manufacturers from using digital technologies to collect data on the shop floor, such as the time and expertise needed to implement data collection and the challenge of handling and interpreting "big data." Many of these issues have been highlighted in the workshop, such as the need for open-source communities, architectures, and infrastructures that deliver formatted PHM data to drive timely, actionable intelligence.

To address many of the information science needs in the PHM community, the PHM4SMS project is currently developing a systems-level test bed composed of networked machines and sensors in an active manufacturing facility. Figure 6-2 provides an overview of the high-level design of this test bed. A major aspect of this effort is the development of a reference implementation that enables manufacturers to collect relevant PHM data from heterogeneous systems on the shop floor without disruption to operations. This research will focus on the protocols and tools needed to communicate data, information, and metrics across the component, sub-system, and system levels for diagnostics and prognostics in manufacturing. They can also enable the prediction of the system-level impacts of events occurring at a single component or sub-system and may enhance process management and control approaches. In addition to standardization activities for PHM, these test bed efforts support several of the roadmap activities identified by the workshop, including PHM Data Format, Taxonomy, and Architecture; Overarching Architecture Framework for PHM with Standards and KPIs; Open-Source Community for PHM; and PHM Infrastructure to Deliver Relevant Timely Information. 


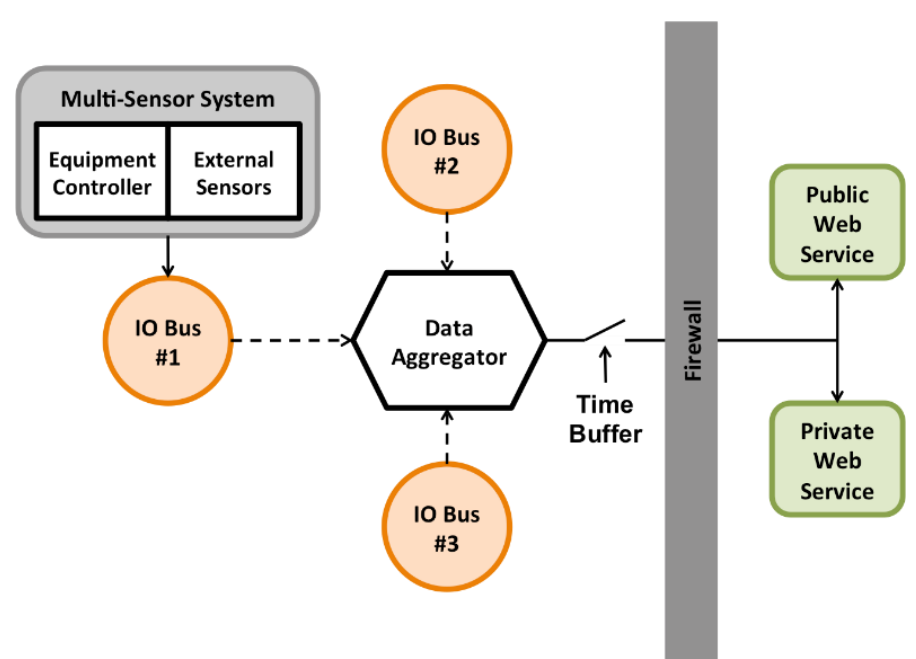

Figure 6-2 - Overview of test bed design to collect and transmit data from various machines and sensors

The systems-level test bed will be a source of data once established. The PHM4SMS project will use this data to generate reference datasets of fabrication and inspection data that may be leveraged to identify useful links for improved diagnostics and process monitoring capabilities. Such research helps develop advanced sensing capabilities, which have been identified as an important roadmap activity during the workshop. The test bed will also enable the development of reference datasets for validation of diagnostic and prognostic standards and technologies. The data generated by the systems-level test bed will also be released directly to the public to support the broader PHM research community. Both of these activities help fill an important need highlighted during the workshop for PHM data, which is often difficult to extract and share to encourage collaboration and further innovation. This data can also support modeling, simulation, and visualization efforts, which are all challenges found for a variety of PHM topics discussed during the workshop.

The data and infrastructure provided by the test bed can also support external efforts to build PHM capabilities. For example, NIST is collaborating with the University of Virginia to develop a hierarchical methodology aimed at decomposing complex manufacturing systems-of-systems into their physical elements and corresponding functional processes to identify the most critical PHM needs to minimize system downtime. This methodology can be potentially verified and validated using the data and information flowing from the test bed. PHM researchers can also use the test bed data to build demonstration applications that can help build a business case for PHM and show the value of this capability to industry. Such activities are necessary to accelerate the development and adoption of PHM for smart manufacturing, which was another crosscutting challenge discussed during the workshop.

\subsection{PHM for Robotics}

Robotics are increasing in their capabilities and range of applications in manufacturing. PHM considerations of a robotic system extend beyond just the robot arm; nearly every robotic system features an end-effector, sensors, safety system(s), supporting/surrounding automation, controller, etc. Robotic systems, especially in smart manufacturing environments, are often marked by complex interactions among these elements. For example, a fault or failure that presents itself as unexpected or inappropriate robot behavior is likely to have resulted not from a robot arm failure, but rather from a failure elsewhere in the system (e.g., sensor failure, controller fault, etc.). NIST's PHM4SMS project is developing test 
methods, metrics, assessment protocols, and reference data sets to support the assessment of condition monitoring, diagnostic, and prognostic techniques within the robotics domain including how degradation impacts key elements of the robot system. This research will be centered around a PHM4Robotics test bed that is currently under construction and expected to be operational and produce its first data sets in 2016.

Several key features stand out as being necessary for a successful PHM system for robotics. NIST research will address the measurement science challenges hindering further development.

- Real-time monitoring or 'quick test' method to identify the health of the robotic system Manufacturers cannot afford long production shutdowns to their lines or workcells. In the trend towards smaller product volumes and increasing product variety, quick testing of reconfigured workcells can be critical to improving productivity and reducing shutdown time. Research in this area will address elements from several priority roadmaps including Identification of PHM Performance Metrics, production of Failure Data for Prognostics and Diagnostics, and PHM Infrastructure to Deliver Relevant Timely Information.

- Closed-loop solution - To achieve maximum OEE, a PHM system needs to efficiently determine the root cause and appropriate remedy (i.e., course of action) of any fault or failure. These actions can be more effectively accomplished in a closed-loop solution (as compared to an open loop solution). Research into such a solution will encompass elements from several priority roadmaps including PHM Data Format Taxonomy, and Architecture; Overarching Architecture Framework for PHM with Standards and KPIs; and PHM Infrastructure to Deliver Relevant Timely Information.

- Intelligent sensing - New, advanced sensing clouds or fusion of existing sensors need to be developed to address complex and/or reconfigurable robotic applications. This research will address some of the elements found in the Advanced Sensors for PHM roadmap. 


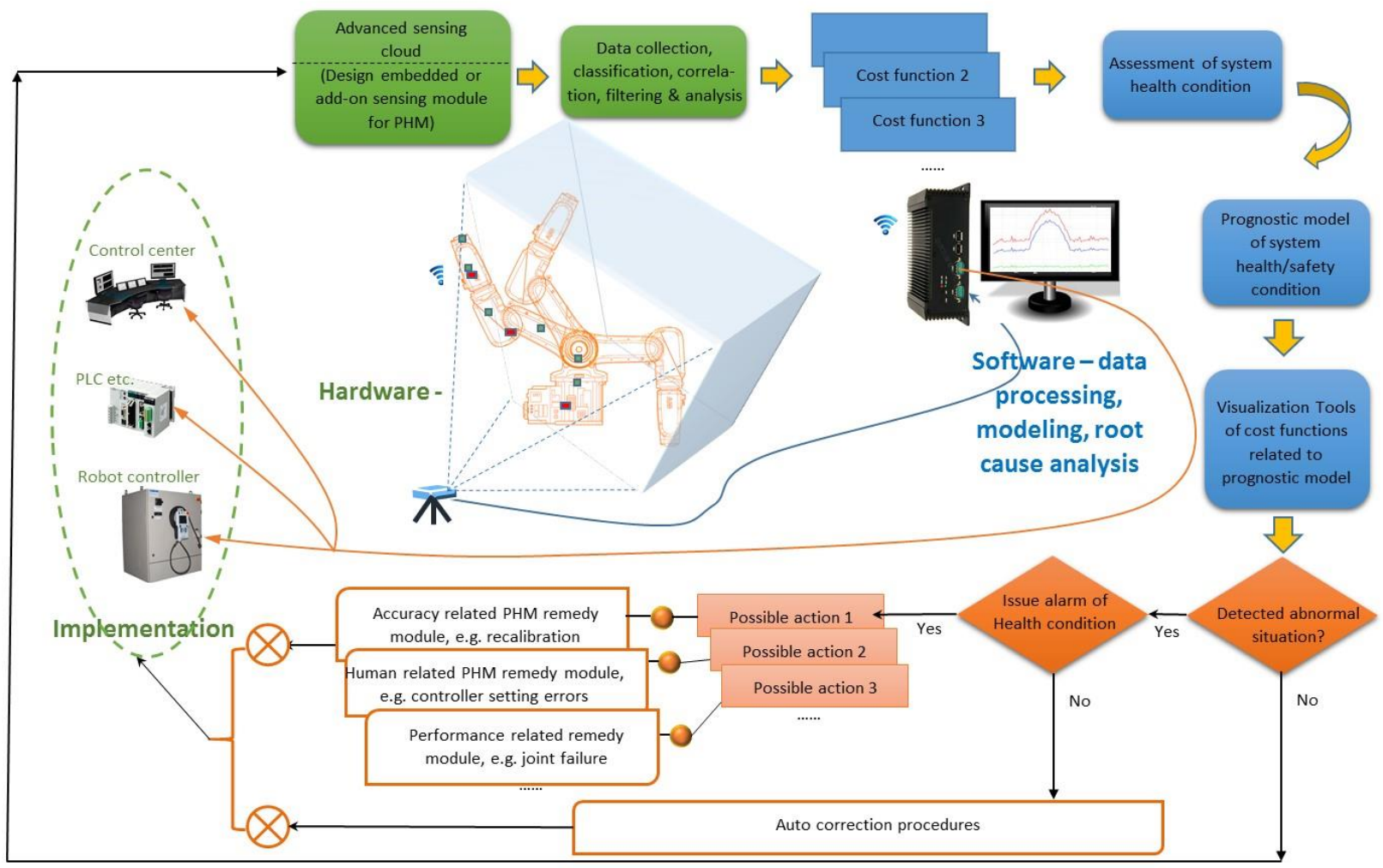

Figure 6-3 - Key Building Blocks of the PHM4Robotics Test Bed

Figure 6-3 presents the key modules of the closed-loop PHM4Robotics test bed. NIST will be developing each one of these modules to serve as the foundation for much of its measurement science research. The development and integration of these modules will enable the design of relevant test methods and use case scenarios to assess PHM operating within robotic systems and enable the verification and validation of external modules. Likewise, the PHM4Robotics test bed will serve as a data production platform to generate reference data sets including those data presenting precursors to various faults and failures. Although the test bed will originally be constructed with a currently-available industrial robot, all of the modules and measurement science will be designed to be robot agnostic. The generation of reference data sets and purposeful focus of the measurement science to be robot agnostic will leverage the Open-Source Community for PHM priority roadmap. This will result in numerous contributions including 1) enabling correlation analyses for root cause fault detection since data will be captured from the component to the system levels, 2) producing reference data from a wide range of sensors, and 3) supporting the development of a standard platform for sensing, data storage, dissemination, etc.

The development and expansion of each module shown in Figure 6-3 will further address elements of the priority roadmaps. For example, as the sensing module is developed, the research team will inventory existing and potentially-relevant sensing technologies that can integrated with industrial robotic systems to promote richer situational awareness (addressing elements of the Advanced Sensors for PHM roadmap). This activity will lead to understanding the raw and output data formats to determine how this information can be appropriately structured and classified for broad use (addressing elements of the PHM Data Format, Taxonomy, and Architecture roadmap). 


\subsection{Summary}

NIST research efforts are aimed at enhancing the industrial competitiveness of U.S. industry within this specific scope of manufacturing PHM. The research highlighted in this section provides a glimpse of both the overall efforts, and their specific status, to inform the PHM community. As progress is made on these efforts, NIST researchers will continue to evolve their plans to meet the needs of industry. 


\section{Appendices}

\section{Appendix A. Contributors}

\begin{tabular}{|c|c|}
\hline Name & Affiliation \\
\hline Brian Antonishek & NIST \\
\hline Peter Beling & University of Virginia \\
\hline Michael Bodach & Boeing \\
\hline Patrick Brown & IMS Center (University of Cincinnati) \\
\hline Carl Byington & Impact Tech/Sikorsky \\
\hline Dan Carnahan & Rockwell Automation \\
\hline Jamie Coble & University of Tennessee \\
\hline Em Delahostria & Rockwell Automation \\
\hline Dave Edstrom & Memex Automation \\
\hline Neil Eklund & Schlumberger \\
\hline Miguel Veregas Franbrana & University of Maryland \\
\hline Kai Goebel & NASA \\
\hline David $\mathrm{He}$ & University of Illinois-Chicago \\
\hline Moneer Helu & NIST \\
\hline Andrew Hess & The Hess PHM Group \\
\hline Peng Y. Huang & Cosen Saws \\
\hline Andy Inman & Toyota \\
\hline Xiaoning Jin & University of Michigan \\
\hline Harry Kekedjian & Ford Motor Company \\
\hline Agnes Klucha & UTC Aerospace Systems \\
\hline Edzel Lapira & Predictronics Corp. \\
\hline Jay Lee & IMS Center, University of Cincinnati \\
\hline Jingshan Li & University of Wisconsin - Madison \\
\hline Yang Li & University of Michigan \\
\hline Linxia Liao & Palo Alto Research Center (PARC) \\
\hline Wentao Luan & University of Maryland \\
\hline Bruce Mahone & SAE International \\
\hline Dipanleus Maily & University of Maryland \\
\hline
\end{tabular}




\begin{tabular}{l|l} 
Name & Affiliation \\
\hline K.C. Morris & NIST \\
\hline Bill Marscher & Mechanical Solutions, Inc. \\
\hline Tom Mooney & Soar Engineering LLC \\
\hline Noah Myrent & Vanderbilt University \\
\hline Chris Nemarich & U.S. Navy Military Sealift Command \\
\hline Dan Oropeza & Johnson Controls Inc. \\
\hline John Oskin & Lockheed Martin \\
\hline Rahul Palnitkar & Sage Clarity \\
\hline Nicholas Propes & Expert Microsystems \\
\hline Karl Reichard & Global Technology Connection \\
\hline Justinian Rosca & Penn State Applied Research Lab \\
\hline Al Salour & Siemens Corporate Technology \\
\hline Peter Sandborn & The Boeing Company \\
\hline David Siegel & University of Maryland \\
\hline Will Sobel & Predictronics Corp. \\
\hline Brinda Thomas & System Insights \\
\hline Jonathan Vance & Tesla Motors \\
\hline Greg Vogl & The Boeing Company \\
\hline Brian A. Weiss & NIST \\
\hline Miguel Zambrana & NIST \\
\hline & University of Maryland \\
\hline
\end{tabular}




\section{Appendix B. Acronyms}

$\begin{array}{ll}\text { CBM } & \text { Condition-based maintenance } \\ \text { CNC } & \text { Computer Numerical Control } \\ \text { DAQ } & \text { Data acquisition } \\ \text { EL } & \text { NIST Engineering Laboratory } \\ \text { EPA } & \text { Environmental Protection Agency } \\ \text { FMECA } & \text { Failure mode, effects, and criticality analysis } \\ \text { HMI } & \text { Human machine interface } \\ \text { HW } & \text { Hardware } \\ \text { ISO } & \text { International Standards Organization } \\ \text { IT } & \text { Information technology } \\ \text { KPI } & \text { Key performance indicator } \\ \text { LRU } & \text { Line replaceable unit } \\ \text { MIMOSA } & \text { Machinery Information Management Open Systems Alliance } \\ \text { MSV } & \text { Models, Simulation, and Visualization } \\ \text { MTConnect } & \text { Manufacturing industry standard to facilitate the organized retrieval of process information from } \\ & \text { numerically controlled machine tools } \\ \text { MTTF } & \text { Mean time to failure } \\ \text { N/A } & \text { Not applicable } \\ \text { NIST } & \text { National Institute of Standards and Technology } \\ \text { NT } & \text { Network } \\ \text { OEE } & \text { Overall equipment effectiveness } \\ \text { OPXC } & \text { Optical Path Crossconnect System } \\ \text { PHM } & \text { Prognostics and Health Management } \\ \text { PHM4SMS } & \text { PHM for Smart Manufacturing Systems } \\ \text { PLC } & \text { Programmable logic controller } \\ \text { RFID } & \text { Radio frequency identification } \\ \text { ROI } & \text { Return on investment } \\ \text { RUL } & \text { Remaining useful life } \\ \text { SCADA/CMMS } & \text { Supervisory Control and Data Acquisition/ Computerized Maintenance Management System } \\ \text { SHM } & \text { Structural Health Management } \\ \text { SMOPAC } & \text { NIST Smart Manufacturing Operations Planning and Control program } \\ \text { STEP-NC } & \text { Machine tool control language that extends the ISO 10303 STEP standards with the machining } \\ & \text { model in ISO 14649 } \\ \text { SW } & \text { Software } \\ \text { TUR } & \text { Test Uncertainty Ratio } \\ \text { WIN } & \text { Windows } \\ & \end{array}$

